\title{
Symplectic embeddings of 4-dim ellipsoids into cubes
}

\author{
David Frenkel And Dorothee Müller
}

Recently, McDuff and Schlenk determined in $\mathrm{MS}$ the function $c_{E B}(a)$ whose value at $a$ is the infimum of the size of a 4-ball into which the ellipsoid $E(1, a)$ symplectically embeds (here, $a \geqslant 1$ is the ratio of the area of the large axis to that of the smaller axis of the ellipsoid). In this paper we look at embeddings into four-dimensional cubes instead, and determine the function $c_{E C}(a)$ whose value at $a$ is the infimum of the size of a 4-cube $C^{4}(A)=$ $D^{2}(A) \times D^{2}(A)$ into which the ellipsoid $E(1, a)$ symplectically embeds (where $D^{2}(A)$ denotes the disc in $\mathbb{R}^{2}$ of area $A$ ). As in the case of embeddings into balls, the structure of the graph of $c_{E C}(a)$ is very rich: for $a$ less than the square $\sigma^{2}$ of the silver ratio $\sigma:=1+\sqrt{2}$, the function $c_{E C}(a)$ turns out to be piecewise linear, with an infinite staircase converging to $\left(\sigma^{2}, \sqrt{\sigma^{2} / 2}\right)$. This staircase is determined by Pell numbers. On the interval $\left[\sigma^{2}, 7 \frac{1}{32}\right]$, the function $c_{E C}(a)$ coincides with the volume constraint $\sqrt{\frac{a}{2}}$ except on seven disjoint intervals, where $c$ is piecewise linear. Finally, for $a \geqslant 7 \frac{1}{32}$, the functions $c_{E C}(a)$ and $\sqrt{\frac{a}{2}}$ are equal.

For the proof, we first translate the embedding problem $E(1, a)$ $\hookrightarrow C^{4}(A)$ to a certain ball packing problem of the ball $B^{4}(2 A)$. This embedding problem is then solved by adapting the method from [MS], which finds all exceptional spheres in blow-ups of the complex projective plane that provide an embedding obstruction.

We also prove that the ellipsoid $E(1, a)$ symplectically embeds into the cube $C^{4}(A)$ if and only if $E(1, a)$ symplectically embeds into the ellipsoid $E(A, 2 A)$. Our embedding function $c_{E C}(a)$ thus also describes the smallest dilate of $E(1,2)$ into which $E(1, a)$ symplectically embeds.

\begin{tabular}{lll}
\hline 1 & Introduction & 767
\end{tabular}

\begin{tabular}{lll}
\hline 1.1 & Statement of the result & 767
\end{tabular}

Partially supported by SNF grant 200020-144432/1. 
\begin{tabular}{lll}
\hline 1.2 & Relations to ECH-capacities & 770
\end{tabular}

\begin{tabular}{|ll|l|l|}
\hline 2 & Proof of Proposition & 1.4 & and equalities (1.1)
\end{tabular}

2.1 Decomposing an ellipsoid into a disjoint union of balls 773

$\begin{array}{lll}2.2 & \text { Representations of balls and polydiscs } & 775\end{array}$

\begin{tabular}{|ll|l|}
\hline 2.3 & Proof of Proposition & 1.4 \\
\hline
\end{tabular}

\begin{tabular}{lll}
\hline 2.4 Proof of equalities (1.1) & 778
\end{tabular}

3 Reduction to a constraint function given by exceptional $\begin{array}{ll}\text { spheres } & \mathbf{7 8 0}\end{array}$

\begin{tabular}{lll}
\hline 4 & Basic observations & 784
\end{tabular}

$\begin{array}{lll}5 & \text { The interval }\left[1, \sigma^{2}\right] & 792\end{array}$

\begin{tabular}{lll}
\hline 5.1 & Preliminaries & 792
\end{tabular}

$\begin{array}{lll}5.2 & \text { The classes } E\left(\alpha_{n}\right) \text { belong to } \mathcal{E} & 795\end{array}$

\begin{tabular}{lll}
\hline 6 & The interval $\left[\sigma^{2}, 6\right]$ & 808
\end{tabular}

\begin{tabular}{lll}
\hline 7 & The interval $[6,8]$ & 826
\end{tabular}

\begin{tabular}{lll}
\hline 7.1 & Preliminaries & 826
\end{tabular}

\begin{tabular}{lll}
\hline 7.2 & The interval $[6,7]$ & 828
\end{tabular}

\begin{tabular}{lll}
\hline 7.3 & The interval $[7,8]$ & 834
\end{tabular}

\begin{tabular}{|ll}
\hline Appendix A & Computer programs \\
\hline
\end{tabular}

A.1 Computing $c$ at a point $a \in\left[6 \frac{1}{8}, 7\right]$

A.2 Computing $c$ on an interval $] 6 \frac{1}{k+1}, 6 \frac{1}{k}$ with $k \in\{1, \ldots, 7\}$ 


\section{Introduction}

\subsection{Statement of the result}

Let $\left(\mathbb{R}^{4}, \omega\right)$ be the Euclidean 4-dimensional space endowed with the canonical symplectic form $\omega=d x_{1} \wedge d y_{1}+d x_{2} \wedge d y_{2}$. Any open subset of $\mathbb{R}^{4}$ is also endowed with $\omega$. Simple examples are the symplectic cylinders $Z(a):=$ $D^{2}(a) \times \mathbb{R}^{2}$ (where $D^{2}(a)$ is the open disc of area $a$ ), the open symplectic ellipsoids

$$
E\left(a_{1}, a_{2}\right)=\left\{\left(x_{1}, y_{1}, x_{2}, y_{2}\right) \in \mathbb{R}^{4}: \frac{\pi\left(x_{1}^{2}+y_{1}^{2}\right)}{a_{1}}+\frac{\pi\left(x_{2}^{2}+y_{2}^{2}\right)}{a_{2}}<1\right\}
$$

and the open polydiscs $P\left(a_{1}, a_{2}\right):=D^{2}\left(a_{1}\right) \times D^{2}\left(a_{2}\right)$. We denote the open ball $E(a, a)$ (of radius $\sqrt{a / \pi})$ by $B(a)$ and the open cube $P(a, a)$ by $C(a)$. Since $D^{2}(a)$ is symplectomorphic to an open square, $D^{2}(a) \times D^{2}(a)$ is indeed symplectomorphic to a cube.

Given two open subsets $U$ and $V$, we say that a smooth embedding $\varphi: U \hookrightarrow V$ is a symplectic embedding if $\varphi$ preserves $\omega$, that is, if $\varphi^{*} \omega=\omega$. In the sequel, we will write $\varphi: U \stackrel{s}{\hookrightarrow} V$ for such an embedding. Since symplectic embeddings are volume preserving, a necessary condition for the existence of a symplectic embedding $U \stackrel{s}{\hookrightarrow} V$ is, of course, $\operatorname{Vol}(U) \leqslant \operatorname{Vol}(V)$, where $\operatorname{Vol}(U):=\frac{1}{2} \int_{U} \omega \wedge \omega$. For volume preserving embeddings, this is the only condition (see e.g. [S1]). For symplectic embeddings, however, the situation is very different, as was detected by Gromov in [G]. Among many other things, he proved

Example 1.1. (Gromov's nonsqueezing Theorem) There exists a symplectic embedding of the ball $B(a)$ into the cylinder $Z(A)$ if and only if $a \leqslant A$.

Notice that the volume of the cylinder $Z(A)$ is infinite, and that for any $a$ the ball $B(a)$ embeds by a linear volume preserving embedding into $Z(A)$. Similarly, we also have

Example 1.2. There exists a symplectic embedding of the ball $B(a)$ into the cube $C(A)$ if and only if $a \leqslant A$.

The above results show that symplectic embeddings are much more special and in some sense "more rigid" than volume preserving embeddings. A next step was to understand this rigidity better. One way of doing this is to fix a 
domain $V \subset \mathbb{R}^{4}$ of finite volume, and to try to determine for each $k \in \mathbb{N}$ the $k$-th packing number

$$
p_{k}(V):=\sup \left\{\frac{k \operatorname{Vol}(B(a))}{\operatorname{Vol}(V)}: \bigsqcup_{k} B(a) \stackrel{s}{\hookrightarrow} V\right\}
$$

Here, $\bigsqcup_{k} B(a)$ is the disjoint union of $k$ equal balls $B(a)$. It follows from Darboux's Theorem that always $p_{k}(V)>0$. If $p_{k}(V)=1$, one says that $V$ admits a full packing by $k$ balls, and if $p_{k}(V)<1$, one says that there is a packing obstruction. Again, it is known that if we would consider volume preserving embeddings instead, then all packing numbers would always be 1 .

In important work by Gromov [G], McDuff-Polterovich [MP], and Biran [B1] all the packing numbers of the 4-ball $B$ and the 4-cube $C$ were determined. The result for $C$ is

\begin{tabular}{ccccccccc}
\hline$k$ & 1 & 2 & 3 & 4 & 5 & 6 & 7 & $\geqslant 8$ \\
\hline$p_{k}$ & $\frac{1}{2}$ & 1 & $\frac{2}{3}$ & $\frac{8}{9}$ & $\frac{9}{10}$ & $\frac{48}{49}$ & $\frac{224}{225}$ & 1 \\
\hline
\end{tabular}

This result shows that, while there is symplectic rigidity for many small $k$, there is no rigidity at all for large $k$.

In order to better understand these numbers, we look at a problem that interpolates the above problem of packing by $k$ equal balls. For $0<a_{1} \leqslant$ $a_{2}$, consider the ellipsoid $E\left(a_{1}, a_{2}\right)$ defined above, and look for the smallest cube $C(A)$ into which $E\left(a_{1}, a_{2}\right)$ symplectically embeds. Since $E\left(a_{1}, a_{2}\right) \stackrel{s}{\hookrightarrow}$ $C(A)$ if and only if $E\left(\lambda a_{1}, \lambda a_{2}\right) \stackrel{s}{\hookrightarrow} C(\lambda A)$, we can always assume that $a_{1}=1$, and therefore study the embedding capacity function

$$
c_{E C}(a):=\inf \{A: E(1, a) \stackrel{s}{\hookrightarrow} C(A)\}
$$

on the interval $[1, \infty[$. It is clear that $c$ is a continuous and nondecreasing function. Since symplectic embeddings are volume preserving and the volumes of $E(1, a)$ and $C(A)$ are $\frac{1}{2} a$ and $A^{2}$ respectively, we must have the lower bound

$$
\sqrt{\frac{a}{2}} \leqslant c(a)
$$

It is not hard to see that $\bigsqcup_{k} B(1) \stackrel{s}{\hookrightarrow} E(1, k)$. Therefore, $\bigsqcup_{k} B(1) \stackrel{s}{\hookrightarrow} C(A)$ whenever $E(1, k) \stackrel{s}{\hookrightarrow} C(A)$. In [M2], McDuff has shown that the converse is also true! Our ellipsoid embedding problem therefore indeed interpolates the 
problem of packing by $k$ equal balls, and we get

$$
p_{k}(C)=\frac{\operatorname{Vol}(E(1, k))}{\operatorname{Vol}\left(C\left(c_{E C}(k)\right)\right)}=\frac{\frac{k}{2}}{\left(c_{E C}(k)\right)^{2}}
$$

First upper estimates for the function $c_{E C}(a)$ were obtained in Chapter 4.4 of [S2] by explicit embeddings of ellipsoids into a cube. These upper estimates also suggested that symplectic rigidity for the problem $E(1, a) \stackrel{s}{\hookrightarrow} C(A)$ should disappear for large $a$.

In this paper, we completely determine the function $c(a):=c_{E C}(a)$. In order to state our main theorem, we introduce two sequences of integers: the Pell numbers $P_{n}$ and the half companion Pell numbers $H_{n}$, which are defined by the recurrence relations

$$
\begin{aligned}
& P_{0}=0, \quad P_{1}=1, \quad P_{n}=2 P_{n-1}+P_{n-2}, \\
& H_{0}=1, \quad H_{1}=1, \quad H_{n}=2 H_{n-1}+H_{n-2} .
\end{aligned}
$$

Thus, $P_{2}=2, P_{3}=5, P_{4}=12, P_{5}=29, \ldots$ and $H_{2}=3, H_{3}=7, H_{4}=17$, $H_{5}=41, \ldots$. The two sequences $\left(\alpha_{n}\right)_{n \geqslant 0}$ and $\left(\beta_{n}\right)_{n \geqslant 0}$ are then defined by

$$
\alpha_{n}:=\left\{\begin{array}{ll}
\frac{2 P_{n+1}^{2}}{H_{n}^{2}} & \text { if } n \text { is even, } \\
\frac{H_{n+1}^{2}}{2 P_{n}^{2}} & \text { if } n \text { is odd; }
\end{array} \quad \beta_{n}:= \begin{cases}\frac{H_{n+2}}{H_{n}} & \text { if } n \text { is even } \\
\frac{P_{n+2}}{P_{n}} & \text { if } n \text { is odd. }\end{cases}\right.
$$

The first terms in these sequences are

$$
\alpha_{0}=2<\beta_{0}=3<\alpha_{1}=\frac{9}{2}<\beta_{1}=5<\alpha_{2}=\frac{50}{9}<\beta_{2}=\frac{17}{3}<\cdots
$$

More generally, for all $n \geqslant 0$,

$$
\cdots<\alpha_{n}<\beta_{n}<\alpha_{n+1}<\beta_{n+1}<\cdots
$$

and both sequences converge to $\sigma^{2}=3+2 \sqrt{2} \cong 5.83$, which is the square of the silver ration $\sigma:=1+\sqrt{2}$. 
Theorem 1.3. (i) On the interval $\left[1, \sigma^{2}\right]$,

$$
c(a)=\left\{\begin{array}{cl}
1 & \text { if } a \in[1,2], \\
\frac{1}{\sqrt{2 \alpha_{n}}} a & \text { if } a \in\left[\alpha_{n}, \beta_{n}\right], \\
\sqrt{\frac{\alpha_{n+1}}{2}} & \text { if } a \in\left[\beta_{n}, \alpha_{n+1}\right],
\end{array}\right.
$$

for all $n \geqslant 0$ (see Figure 1.1).

(ii) On the interval $\left[\sigma^{2}, 7 \frac{1}{32}\right]$ we have $c(a)=\sqrt{\frac{a}{2}}$ except on seven disjoint intervals, where $c$ is piecewise linear (see Figure 1.2).

(iii) For $a \geqslant 7 \frac{1}{32}$ we have $c(a)=\sqrt{\frac{a}{2}}$.

The proof of (i) is given in Corollary 5.2, a more detailed statement as well as the proof of (ii) are given in Theorem 7.2. while the proof of (iii) is given in Lemma 4.1 and Proposition 7.7

A similar result has been previously obtained by McDuff-Schlenk in [MS] for the embedding problem $E(1, a) \stackrel{s}{\hookrightarrow} B(A)$. These two results show that the structure of symplectic rigidity can be very rich.

For further results on packings of various symplectic manifolds by balls and ellipsoids we refer to [B2], $\mathrm{BuH1}],[\mathrm{BuH} 2,[\mathrm{BuP}],[\mathrm{LMS}]$ and [O].

\subsection{Relations to ECH-capacities}

There is a more combinatorial (but non-explicit) way of describing the embedding function $c_{E C}(a)$. Indeed, in [H1], Hutchings used his embedded contact homology to construct for each domain $U \subset \mathbb{R}^{4}$ a sequence of symplectic capacities $c_{E C H}^{k}(U)$, which for the ellipsoid $E(a, b)$ and the polydisc $P(a, b)$ are as follows.

Form the sequence $N_{E}(a, b)$ by arranging all numbers of the form $m a+n b$ with $m, n \geqslant 0$, in nondecreasing order (with multiplicities). Then for $k \geqslant 0$, the $k$-th ECH-capacity $c_{E C H}^{k}(E(a, b))$ is the $(k+1)$-th entry of $N_{E}(a, b)$. For instance, $c_{E C H}(E(1,1))=\{0,1,1,2,2,2,3,3,3,3,4, \ldots\}$.

Moreover, for polydiscs,

$$
c_{E C H}^{k}(P(a, b))=\min \{a m+b n: m, n \in \mathbb{N} ;(m+1)(n+1) \geqslant k+1\} .
$$

There exists a canonical way to decompose an ellipsoid $E(a, b)$ with $\frac{a}{b}$ rational into a finite disjoint union of balls $B(a, b):=\sqcup_{i} B\left(w_{i}\right)$ with weights $w_{i}$ 


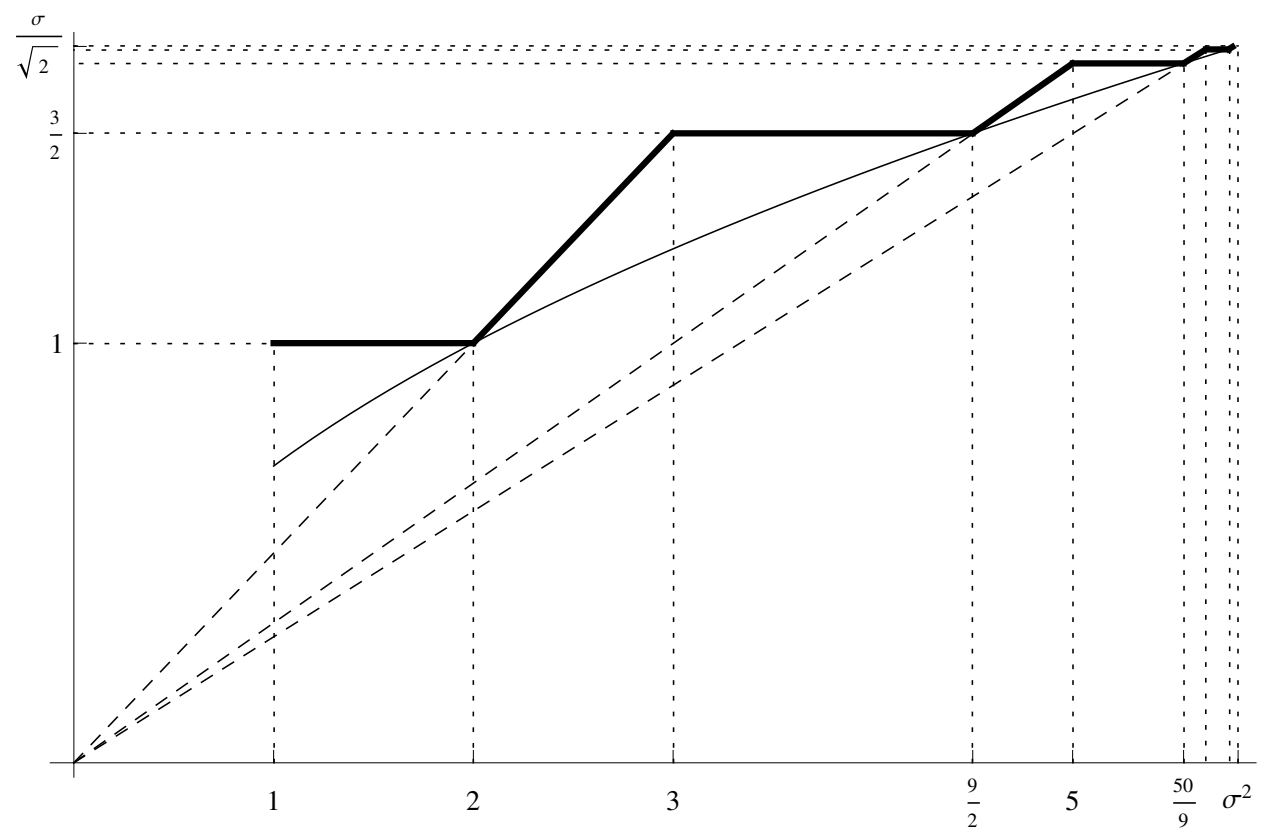

Figure 1.1: The graph of $c$ on the interval $\left[1, \sigma^{2}\right]$.

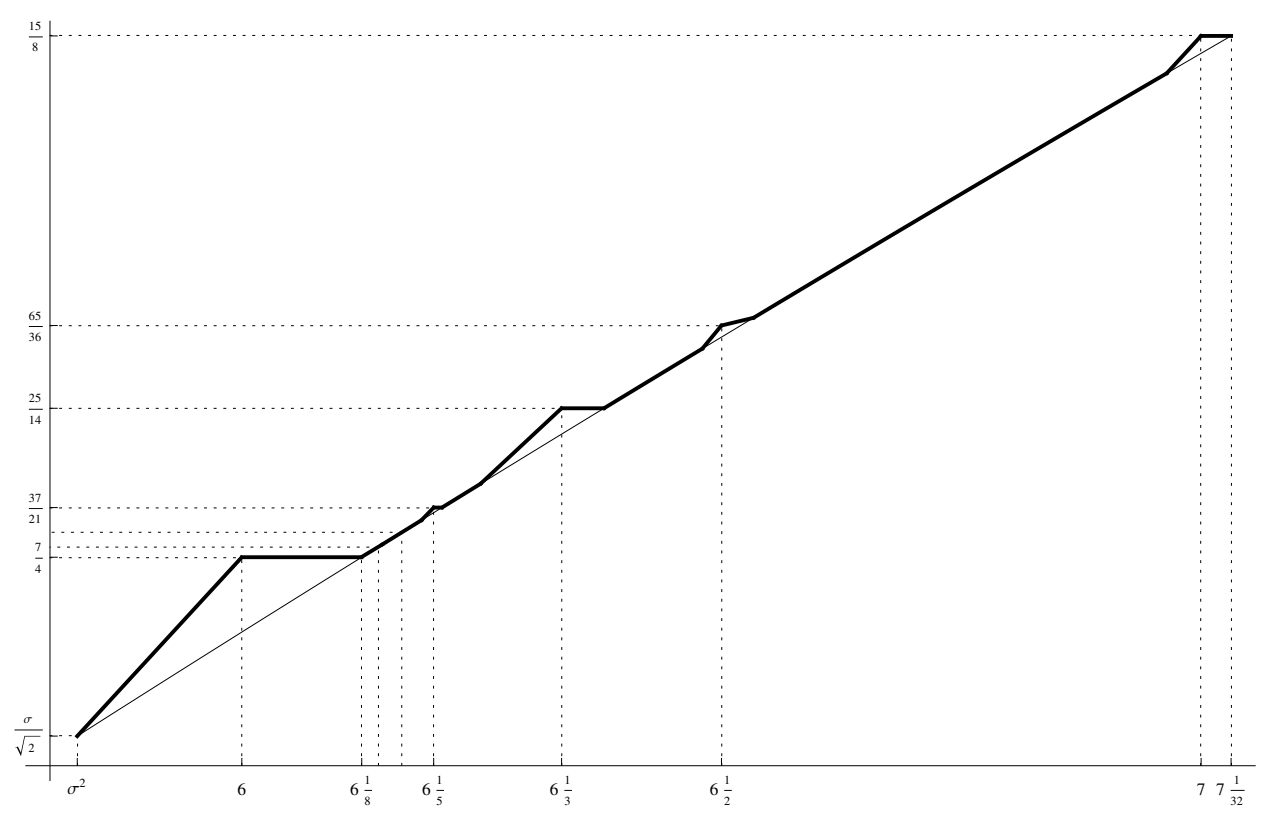

Figure 1.2: The graph of $c$ on the interval $\left[\sigma^{2}, 7 \frac{1}{32}\right]$. 
related to the continued fraction expansion of $\frac{a}{b}$. We shall explain this decomposition in more detail and prove the following proposition in the next section.

Proposition 1.4. Let $a, b, c, d>0$ with $\frac{a}{b}$ rational. Then there exists a symplectic embedding $E(a, b) \hookrightarrow P(c, d)$ if and only if there exists a symplectic embedding

$$
B(a, b) \bigsqcup B(c) \bigsqcup B(d) \hookrightarrow B(c+d)
$$

Hutchings showed in Corollary 11 of [H2] how Proposition 1.4 implies that ECH-capacities form a complete set of invariants for the problem of symplectically embedding an ellipsoid into a polydisc:

Corollary 1.5. There exists a symplectic embedding $E(a, b) \hookrightarrow P(c, d)$ if and only if $c_{E C H}^{k}(E(a, b)) \leqslant c_{E C H}^{k}(P(c, d))$ for all $k \geqslant 0$.

It seems to be hard to derive Theorem 1.3 from Corollary 1.5 or vice-versa.

As a further corollary we obtain

Corollary 1.6. The ellipsoid $E(1, a)$ symplectically embeds into the cube $C(A)$ if and only if $E(1, a)$ symplectically embeds into the ellipsoid $E(A, 2 A)$.

Proof. By Corollary 1.5, $E(1, a)$ symplectically embeds into $C(A)$ if and only if $c_{E C H}^{k}(E(1, a)) \leqslant c_{E C H}^{k}(C(A))$ for all $k \geqslant 0$. By McDuff's proof of the Hofer Conjecture [M3], $E(1, a)$ symplectically embeds into $E(A, 2 A)$ if and only if $c_{E C H}^{k}(E(1, a)) \leqslant c_{E C H}^{k}(E(A, 2 A))$ for all $k \geqslant 0$. The corollary now follows from the remark on page 8098 in [H2], that says that for all $k \geqslant 0$

$$
c_{E C H}^{k}(E(1,2))=c_{E C H}^{k}(C(1)) .
$$

For the easy proof, we refer to Section 2 .

Remark 1.7. Recall that the ECH-capacities of $B(1)$ and $C(1)$ (or $E(1,2)$ ) are

$$
\begin{aligned}
& c_{E C H}(B(1))=\left(0^{\times 1}, 1^{\times 2}, 2^{\times 3}, 3^{\times 4}, 4^{\times 5}, 5^{\times 6}, 6^{\times 7}, 7^{\times 8}, 8^{\times 9}, 9^{\times 10}, \ldots\right), \\
& c_{E C H}(C(1))=\left(0^{\times 1}, 1^{\times 1}, 2^{\times 2}, 3^{\times 2}, 4^{\times 3}, 5^{\times 3}, 6^{\times 4}, 7^{\times 4}, 8^{\times 5}, 9^{\times 5}, \ldots\right) .
\end{aligned}
$$

One sees that the sequence $c_{E C H}(C(1))$ is obtained from $c_{E C H}(B(1))$ by some sort of doubling. This is reminiscent to the doubling in the definition of 
the Pell numbers: The Fibonacci and Pell numbers are defined recursively by

$$
F_{n+1}=F_{n}+F_{n-1}, \quad P_{n+1}=2 P_{n}+P_{n-1},
$$

and while the Fibonacci numbers determine the infinite stairs of the function $c_{E B}(a)$ for $a \leqslant \tau^{4}$ (with $\tau$ the golden ratio, see [MS]), the Pell numbers determine the infinite stairs of the function $c_{E C}(a)$ for $a \leqslant \sigma^{2}$. This reminiscence may, however, be a coincidence. Indeed, for the ellipsoid $E(1,3)$ the sequence

$$
c_{E C H}\left(E(1,3)=\left(0^{\times 1}, 1^{\times 1}, 2^{\times 1}, 3^{\times 2}, 4^{\times 2}, 5^{\times 2}, 6^{\times 3}, 7^{\times 3}, 8^{\times 3}, 9^{\times 4}, \ldots\right)\right.
$$

is obtained from $c_{E C H}(B(1))$ by some sort of trippling, but the beginning of the function describing the embedding problem $E(1, a) \stackrel{s}{\hookrightarrow} E(A, 3 A)$ seems not to be given in terms of numbers defined by $G_{n+1}=3 G_{n}+G_{n-1}$.

Acknowledgments. We wish to sincerely thank Felix Schlenk for his precious help and encouragement during the whole project and Régis Straubhaar for his help with all the computer issues. The present work is part of the author's activities within CAST, a Research Network Program of the European Science Foundation.

\section{Proof of Proposition 1.4 and equalities (1.1)}

In Section 2.1. we explain the canonical decomposition of $E(1, a)$ with $a \in \mathbb{Q}$ into a disjoint union of balls. We then prove Proposition 1.4 in Sections 2.2 and 2.3. and in Section 2.4 we prove equalities (1.1).

\subsection{Decomposing an ellipsoid into a disjoint union of balls}

In [M2], McDuff showed the following theorem.

Theorem 2.1. (McDuff [M2]) Let $a, b>0$ be two rational numbers. Then, there exists a finite sequence $\left(w_{1}, \ldots, w_{M}\right)$ of rational numbers such that the closed ellipsoid $\bar{E}(a, b)$ symplectically embeds into the ball $B(A)$ if and only if the disjoint union of balls $\sqcup_{i} \bar{B}\left(w_{i}\right)$ symplectically embed into $B(A)$.

The disjoint union $\sqcup_{i} \bar{B}\left(w_{i}\right)$ is then denoted by $\bar{B}(a, b)$. Following [MS], we will now explain one way to compute the weights $w_{1}, \ldots, w_{M}$ in this decomposition. Notice that in [M2], the weights of the balls $B\left(w_{i}\right)$ are defined in a slightly different way. The proof that these weights agree with the weight expansion of $a$ defined now can be found in the Appendix of [MS]. 
Definition 2.2. Let $a=\frac{p}{q} \geqslant 1$ be a rational number written in lowest terms. The weight expansion of $a$ is the finite sequence $w(a):=\left(w_{1}, \ldots, w_{M}\right)$ defined recursively by

- $w_{1}=1$, and $w_{n} \geqslant w_{n+1}>0$ for all $n$;

- if $w_{i}>w_{i+1}=\cdots=w_{n}$ (where we set $\left.w_{0}:=a\right)$, then $w_{n+1}=\left\{\begin{array}{cl}w_{n} & \text { if } w_{i+1}+\cdots+w_{n+1}=(n-i+1) w_{i+1} \leqslant w_{i} \\ w_{i}-(n-i) w_{i+1} & \text { otherwise }\end{array}\right.$

- the sequence stops at $w_{n}$ if the above formula gives $w_{n+1}=0$.

Remark 2.3. If we regard this weight expansion as consisting of $N+1$ blocks on which the $w_{i}$ are constant, that is

$$
w(a)=(\underbrace{1, \ldots, 1}_{l_{0}}, \underbrace{x_{1}, \ldots, x_{1}}_{l_{1}}, \ldots, \underbrace{x_{N}, \ldots, x_{N}}_{l_{N}})=\left(1^{\times l_{0}}, x_{1}^{\times l_{1}}, \ldots, x_{N}^{\times l_{N}}\right),
$$

then $x_{1}=a-l_{0}<1$, and if we set $x_{0}=1$, then for all $2 \leqslant i \leqslant N, x_{i}=x_{i-2}-$ $l_{i-1} x_{i-1}$. Moreover, the lengths of the blocks give the continued fraction of $a$ since

$$
a=l_{0}+\frac{1}{l_{1}+\frac{1}{l_{2}+\frac{1}{\ddots+\frac{1}{l_{N}}}}}=:\left[l_{0} ; l_{1}, \ldots, l_{N}\right] \text {. }
$$

Example 2.4. The weight expansion of $\frac{25}{9}$ is $\left(1^{\times 2}, \frac{7}{9}^{2} \frac{2}{9}^{\times 3}, \frac{1}{9}^{\times 2}\right)$. The continued fraction expansion of $\frac{25}{9}$ is thus $[2 ; 1,3,2]$. Notice that we also have

$$
\frac{25}{9}=2 \cdot 1^{2}+\left(\frac{7}{9}\right)^{2}+3 \cdot\left(\frac{2}{9}\right)^{2}+2 \cdot\left(\frac{1}{9}\right)^{2} .
$$

This is no accident and is best explained geometrically as in Figure 2.1. The general result is stated in the next lemma.

Lemma 2.5. (McDuff-Schlenk [MS], Lemma 1.2.6) Let $a=\frac{p}{q} \geqslant 1$ be a rational number with $p, q$ relatively prime, and let $w:=w(a)=\left(w_{1}, \ldots, w_{M}\right)$ be its weight expansion. Then

(i) $w_{M}=\frac{1}{q}$;

(ii) $\sum w_{i}^{2}=\langle w, w\rangle=a$; 


\begin{tabular}{|c|c|c|c|c|}
\hline & & 2 & 2 & 2 \\
\hline 9 & 9 & & 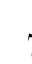 & \\
\hline
\end{tabular}

Figure 2.1: The weight expansion of $\frac{25}{9}$.

(iii) $\sum w_{i}=a+1-\frac{1}{q}$.

\subsection{Representations of balls and polydiscs}

In the proof of Proposition 1.4. we shall use certain ways of representing open and closed balls and open polydiscs. Recall that $B(a)$ is the open ball in $\mathbb{R}^{4}$ of capacity $a=\pi r^{2}$, and that $P(a, b)=D^{2}(a) \times D^{2}(b)$, where $D^{2}(a)$ is the open disc in $\mathbb{R}^{2}$ of area $a$.

2.2.1. Representations as products. Denote by $\square(a, b)$ the open square ] $0, a[\times] 0, b\left[\right.$ in $\mathbb{R}^{2}$. Since $D^{2}(a)$ is symplectomorphic to the open square ] $0, a[\times] 0,1[$, the polydisc $P(a, b)$ is symplectomorphic to

$$
\square(a, b) \times \square(1,1) \subset \mathbb{R}^{2}(x) \times \mathbb{R}^{2}(y) .
$$

Next, consider the simplex

$$
\triangle(a):=\left\{\left(x_{1}, x_{2}\right) \in \mathbb{R}^{2}(x): 0<x_{1}, x_{2} ; x_{1}+x_{2}<a\right\} .
$$

Then $B(a)$ is symplectomorphic to the product

$$
\triangle(a) \times \square(1,1) \subset \mathbb{R}^{2}(x) \times \mathbb{R}^{2}(y),
$$

see [T] and Remark 9.3.1 of [S2].

2.2.2. Representations by the Delzant polytope. As before, denote by $\omega_{S F}$ the Study-Fubini form on the complex projective plane $\mathbb{C} P^{2}$, normalized by $\int_{\mathbb{C} P^{1}} \omega_{S F}=1$. We write $\mathbb{C} P^{2}(a)$ for $\left(\mathbb{C} P^{2}, a \omega_{S F}\right)$. Its affine part $\mathbb{C} P^{2} \backslash \mathbb{C} P^{1}$ is symplectomorphic to the open ball $B(a)$. (Indeed, for $a=\pi$, the embedding

is symplectic.)

$$
z=\left(z_{1}, z_{2}\right) \mapsto\left[z_{1}: z_{2}: \sqrt{1-|z|^{2}}\right]
$$



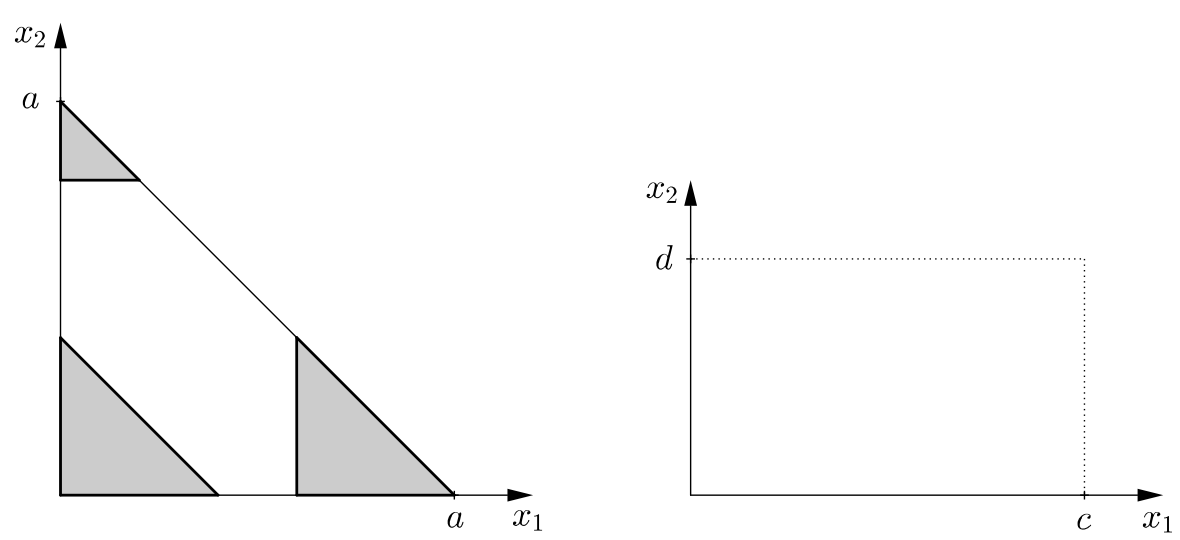

Figure 2.2: Closed balls in $\mathbb{C} P^{2}(a)$ and the moment image of $P(c, d)$.

The image of the moment map of the usual $T^{2}$-action on $\mathbb{C} P^{2}(a)$ is the closed triangle $\overline{\triangle(a)}$. For $b<a$, the preimage of $\overline{\triangle(b)} \subset \overline{\triangle(a)}$ is symplectomorphic to $\bar{B}(b)$. By precomposing the torus action with suitable linear torus automorphisms, one sees that also the closed triangles based at the other two corners of $\overline{\triangle(a)}$ correspond to closed balls in $\mathbb{C} P^{2}(a)$. We refer to $[\mathrm{K}]$ for details.

The image of the moment map of the usual $T^{2}$-action on $\mathbb{C}^{2}$ maps the polydisc $P(c, d)$ to the rectangle $\left[0, c\left[\times\left[0, d\left[\subset \mathbb{R}^{2}(x)\right.\right.\right.\right.$.

\subsection{Proof of Proposition 1.4}

Let now $a, b, c, d>0$ with $\frac{a}{b}$ rational. We need to show that

$$
E(a, b) \stackrel{s}{\hookrightarrow} P(c, d) \Longleftrightarrow B(a, b) \sqcup B(c) \sqcup B(d) \stackrel{s}{\hookrightarrow} B(c+d) .
$$

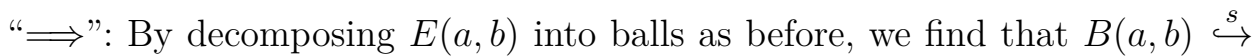
$P(c, d)$, (see also [M2]). Fix $\varepsilon>0$. Then also $(1-\varepsilon) \bar{B}(a, b) \stackrel{s}{\hookrightarrow} P(c, d)$. Now represent the open balls $B(c), B(d), B(c+d)$ and the polydisc as in Section 2.2.1 above. We then read off from Figure 2.3 that

$$
(1-\varepsilon) \bar{B}(a, b) \sqcup B(c) \sqcup B(d) \stackrel{s}{\hookrightarrow} B(c+d) .
$$

This holds for every $\varepsilon>0$. In view of [M1] we then also find a symplectic embedding $B(a, b) \sqcup B(c) \sqcup B(d) \stackrel{s}{\hookrightarrow} B(c+d)$. 


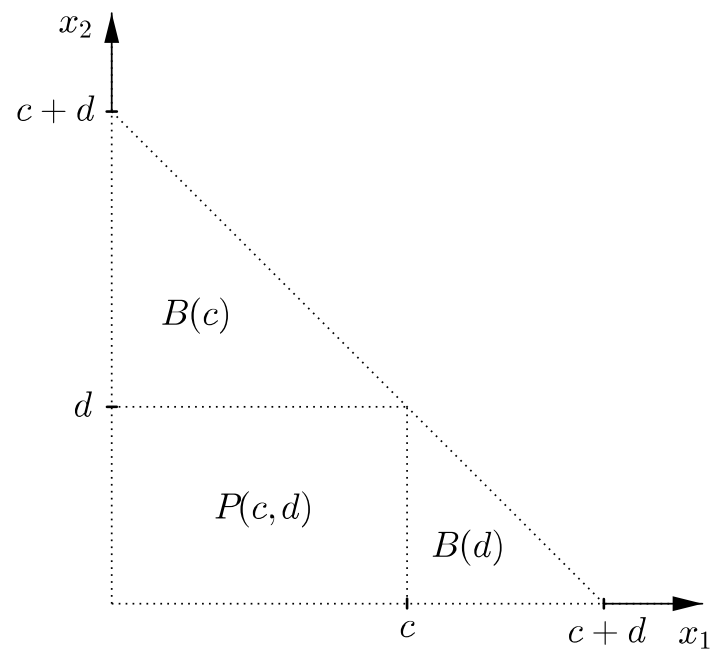

Figure 2.3: $(1-\varepsilon) \bar{B}(a, b) \sqcup B(c) \sqcup B(d) \stackrel{s}{\hookrightarrow} B(c+d)$. Then

"£" : Assume now that $B(a, b) \sqcup B(c) \sqcup B(d) \stackrel{s}{\hookrightarrow} B(c+d)$. Fix $\varepsilon>0$.

$$
(1-\varepsilon) \bar{B}(a, b) \sqcup \bar{B}(c-\varepsilon) \sqcup \bar{B}(d-\varepsilon) \stackrel{s}{\hookrightarrow} \mathbb{C} P^{2}(c+d) .
$$

According to M1, the space of symplectic embeddings of $\bar{B}(c-\varepsilon) \sqcup \bar{B}(d-$ $\varepsilon)$ into $\mathbb{C} P^{2}(c+d)$ is connected. Any such isotopy extends to an ambient symplectic isotopy of $\mathbb{C} P^{2}(c+d)$. In view of this and by Section 2.2.2 we can thus assume that the balls $\bar{B}(c-\varepsilon)$ and $\bar{B}(d-\varepsilon)$ lie in $\mathbb{C} P^{2}(c+d)$ as shown in Figure 2.4

The image of the balls $(1-\varepsilon) \bar{B}(a, b)$ must then lie over the gray shaded closed region. However, since the balls $\bar{B}(c-\varepsilon)$ and $\bar{B}(d-\varepsilon)$ are closed, the image of $(1-\varepsilon) \bar{B}(a, b)$ cannot touch the upper horizontal or the right vertical boundary of the gray shaded region. Moreover, according to Remark 2.1.E of [MP] we can assume that this image lies in the affine part of $\mathbb{C} P^{2}(c+d)$, i.e., the image of the balls $(1-\varepsilon) \bar{B}(a, b)$ lies over the gray shaded region deprived from the dark segment, and hence, by Section 2.2.2 in $P(c+\varepsilon, d+$ $\varepsilon)$. We may suppose from the start that $c, d \geqslant 1$. Then $P(c+\varepsilon, d+\varepsilon) \subset(1+$ ह) $P(c, d)$. We have thus found a symplectic embedding $(1-\varepsilon) \bar{B}(a, b) \stackrel{s}{\hookrightarrow}(1+$ ह) $P(c, d)$. It is shown in Theorem 1.5 of [M2] that then also $(1-\varepsilon) \bar{E}(a, b) \stackrel{s}{\hookrightarrow}$ 


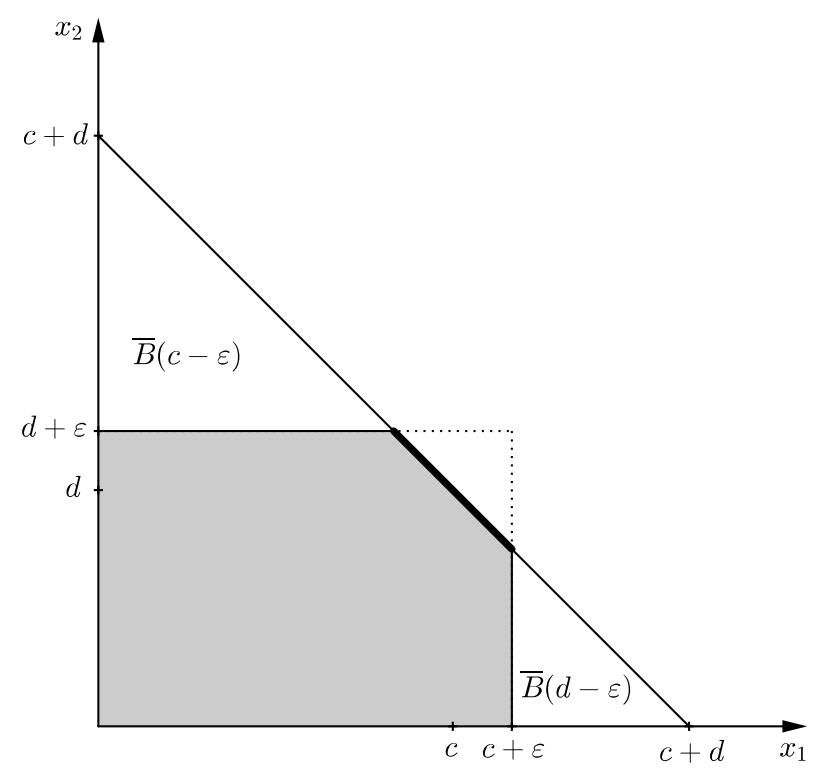

Figure 2.4: How $\bar{B}(c-\varepsilon)$ and $\bar{B}(d-\varepsilon)$ lie in $\mathbb{C} P^{2}(c+d)$.

$(1+\varepsilon) P(c, d)$. Hence

$$
\frac{1-\varepsilon}{1+\varepsilon} \bar{E}(a, b) \stackrel{s}{\hookrightarrow} P(c, d) .
$$

It now follows again from [M2] that $E(a, b) \stackrel{s}{\hookrightarrow} P(c, d)$. (To be precise, M2] considers embeddings of ellipsoids into open balls; however, the same arguments work for embeddings of ellipsoids into polydiscs.)

\subsection{Proof of equalities (1.1)}

Lemma 2.6. For all $k \geqslant 0$,

$$
c_{E C H}^{k}(E(1,2))=c_{E C H}^{k}(C(1)) .
$$

Proof. We will prove that $c_{E C H}^{k}(E(1,2))$ and $c_{E C H}^{k}(C(1))$ are both equal to the unique integer $d$ such that

$$
\left\lfloor\frac{d+1}{2}\right\rfloor\left\lceil\frac{d+1}{2}\right\rceil \leqslant k<\left\lfloor\frac{d+2}{2}\right\rfloor\left\lceil\frac{d+2}{2}\right\rceil .
$$


For $c_{E C H}^{k}(E(1,2))$, this follows from the fact that the number

$$
\sharp\left\{(m, n) \in \mathbb{N}_{0}^{2}: m+2 n \leqslant d\right\}
$$

of pairs of nonnegative integers $(m, n)$ such that $m+2 n \leqslant d$ is equal to $\left\lfloor\frac{d+2}{2}\right\rfloor\left\lceil\frac{d+2}{2}\right\rceil$. This, in turn, can easily be deduced from the identities

$$
\sharp\left\{(m, n) \in \mathbb{N}_{0}^{2}: m+2 n=2 l\right\}=\sharp\left\{(m, n) \in \mathbb{N}_{0}^{2}: m+2 n=2 l+1\right\}=l+1 .
$$

On the other hand, we have

$$
c_{E C H}^{k}(C(1))=c_{E C H}^{k}(P(1,1))=\min \{m+n:(m+1)(n+1) \geqslant k+1\}
$$

Fix a nonnegative integer $k$. Let $m_{0}, n_{0} \in \mathbb{N}_{0}$ be two nonnegative integers such that

$$
m_{0}+n_{0}=\min \{m+n:(m+1)(n+1) \geqslant k+1\}
$$

Without loss of generality, $m_{0} \geqslant n_{0}$. Moreover, we can always take $m_{0}, n_{0}$ such that $m_{0}-n_{0} \in\{0,1\}$. Indeed, assume that $m_{0}=n_{0}+c$ with $c \geqslant 2$. Then for $m_{0}^{\prime}=m_{0}-1$ and $n_{0}^{\prime}=n_{0}+1$, we get

$$
\begin{aligned}
\left(m_{0}^{\prime}+1\right)\left(n_{0}^{\prime}+1\right) & =m_{0}\left(n_{0}+2\right)=\left(n_{0}+c\right)\left(n_{0}+2\right)=n_{0}^{2}+(c+2) n_{0}+2 c \\
& >n_{0}^{2}+(c+2) n_{0}+c+1=\left(n_{0}+c+1\right)\left(n_{0}+1\right) \\
& =\left(m_{0}+1\right)\left(n_{0}+1\right) \geqslant k+1 .
\end{aligned}
$$

Thus $\left(m_{0}^{\prime}, n_{0}^{\prime}\right)$ also realizes the minimum. Now, if $m_{0}+n_{0}$ is even, then $m_{0}=$ $n_{0}$ and we have to show that

$$
\begin{aligned}
\left\lfloor\frac{2 m_{0}+1}{2}\right\rfloor\left\lceil\frac{2 m_{0}+1}{2}\right\rceil & =m_{0}\left(m_{0}+1\right) \leqslant k \\
& <\left(m_{0}+1\right)^{2}=\left\lfloor\frac{2 m_{0}+2}{2}\right\rfloor\left\lceil\frac{2 m_{0}+2}{2}\right\rceil
\end{aligned}
$$

The first inequality follows from the minimality of $m_{0}+n_{0}$ while the second one follows from the fact that $\left(m_{0}+1\right)\left(n_{0}+1\right) \geqslant k+1$. The case $m_{0}+n_{0}$ odd is treated similarly. 


\section{Reduction to a constraint function given by exceptional spheres}

In this section we explain how the function $c(a)$ can be described by the volume constraint $\sqrt{\frac{a}{2}}$ and the constraints coming from certain exceptional spheres in blow-ups of $\mathbb{C} P^{2}$. Since the function $c$ is continuous, it suffices to determine $c$ for each rational $a \geqslant 1$. The starting point is the following lemma, which is a special case of Proposition 1.4 .

Lemma 3.1. Let $a \geqslant 1$ be a rational number with weight expansion $w(a)=$ $\left(w_{1}, \ldots, w_{M}\right)$ and $A>0$. Then the ellipsoid $E(1, a)$ embeds symplectically into the cube $C(A)$ if and only if there is a symplectic embedding

$$
B(A) \sqcup B(A) \sqcup_{i} B\left(w_{i}\right) \stackrel{s}{\hookrightarrow} B(2 A) .
$$

With this lemma, we have converted the problem of embedding an ellipsoid into a cube to the problem of embedding a disjoint union of balls into a ball. In [MP, the problem of embedding $k$ disjoint balls into a ball was reduced to the question of understanding the symplectic cone of the $k$-fold blow-up $X_{k}$ of $\mathbb{C} P^{2}$. Let $L:=\left[\mathbb{C} P^{1}\right] \in H_{2}\left(X_{k}, \mathbb{Z}\right)$ be the class of a line, let $E_{1}, \ldots, E_{k} \in$ $H_{2}\left(X_{k}, \mathbb{Z}\right)$ be the homology classes of the exceptional divisors, and denote by $l, e_{1}, \ldots, e_{k} \in H^{2}\left(X_{k}, \mathbb{Z}\right)$ their Poincaré duals. Let $-K:=3 L-\sum E_{i}$ be the anti-canonical divisor of $X_{k}$, and define the corresponding symplectic cone $\mathcal{C}_{K}\left(X_{k}\right) \subset H^{2}\left(X_{k}, \mathbb{Z}\right)$ as the set of classes represented by symplectic forms $\omega$ with first Chern class $c_{1}(M, \omega)=-K$.

Theorem 3.2. (McDuff-Polterovich [MP]) The union $\sqcup_{i=1}^{k} \bar{B}\left(w_{i}\right)$ embeds into the ball $B(\mu)$ or into $\mathbb{C} P^{2}(\mu)$ if and only if $\mu l-\sum w_{i} e_{i} \in \mathcal{C}_{K}\left(X_{k}\right)$.

To understand $\mathcal{C}_{K}\left(X_{k}\right)$, we define as in $\mathrm{MS}$ the following set $\mathcal{E}_{k} \subset$ $H_{2}\left(X_{k}\right)$.

Definition 3.3. $\mathcal{E}_{k}$ is the set consisting of $(0 ;-1,0, \ldots, 0)$ and of all tuples $(d ; m):=\left(d ; m_{1}, \ldots, m_{k}\right)$ with $d \geqslant 0$ and $m_{1} \geqslant \cdots \geqslant m_{k} \geqslant 0$ such that the class $E_{(d ; m)}:=d L-\sum m_{i} E_{i} \in H_{2}\left(X_{k}\right)$ is represented in $X_{k}$ by a symplectically embedded sphere of self-intersection -1 .

We will often write $\mathcal{E}$ instead of $\mathcal{E}_{k}$ if there is no danger of confusion. We then have the following description of $\mathcal{C}_{K}\left(X_{k}\right)$. 
Proposition 3.4. (Li-Li [LiLi], Li-Liu [LiLiu]

$$
\mathcal{C}_{K}\left(X_{k}\right)=\left\{\alpha \in H^{2}\left(X_{k}\right): \alpha^{2}>0, \alpha(E)>0, \forall E \in \mathcal{E}_{k}\right\}
$$

In order to give a characterization of the set $\mathcal{E}_{k}$, we need the following definition as in $[\mathrm{MS}$.

Definition 3.5. A tuple $(d ; m):=\left(d ; m_{1}, \ldots m_{k}\right)$ is said to be ordered if the $m_{i}$ are in nonincreasing order. The Cremona transform of an ordered tuple $(d ; m)$ is

$$
\left(2 d-m_{1}-m_{2}-m_{3} ; d-m_{2}-m_{3}, d-m_{1}-m_{3}, d-m_{1}-m_{2}, m_{4}, \ldots, m_{k}\right)
$$

A Cremona move of a tuple $(d ; m)$ is the composition of the Cremona transform of $(d ; m)$ with any permutation of the new obtained vector $m$.

Proposition 3.6. (McDuff-Schlenk [MS], Proposition 1.2.12 and Remark 3.3.1)

(i) $\operatorname{All}(d ; m) \in \mathcal{E}_{k}$ satisfy the two Diophantine equations

$$
\begin{aligned}
& \sum m_{i}=3 d-1 \\
& \sum m_{i}^{2}=d^{2}+1
\end{aligned}
$$

(ii) For all distinct $(d ; m),\left(d^{\prime} ; m^{\prime}\right) \in \mathcal{E}_{k}$ we have

$$
\sum m_{i} m_{i}^{\prime} \leqslant d d^{\prime}
$$

(iii) A tuple $(d ; m)$ belongs to $\mathcal{E}_{k}$ if and only if $(d ; m)$ satisfies the Diophantine equations in $(i)$ and $(d ; m)$ can be reduced to $(0 ;-1,0, \ldots, 0)$ by repeated Cremona moves.

Remark 3.7. Working directly with Lemma 3.1. Theorem 3.2 and Proposition 3.4 we find, as in [MS], that the only constraints for an embedding $E(1, a) \stackrel{s}{\hookrightarrow} C(A)$ are $A \geqslant \sqrt{\frac{a}{2}}$ and, for each class $(d ; m) \in \mathcal{E}_{k}$,

$$
2 A d \geqslant\left(m_{1}+m_{2}\right) A+\left\langle\left(m_{3}, \ldots, m_{k}\right), w(a)\right\rangle .
$$

One can start from here and use Proposition 3.6 to prove Theorem 1.3 . The analysis becomes, however, rather awkward, since the unknown $A$ appears on both sides of (3.1). 
To improve the situation, we shall apply a base change of $H_{2}\left(X_{k}\right)$, and express the elements of $\mathcal{E}$ in a new basis. Consider the product $S^{2} \times S^{2}$ (whose affine part is a cube), and form the $M$-fold (topological) blow-up $X_{M}\left(S^{2} \times S^{2}\right)$. A basis of $H_{2}\left(X_{M}\left(S^{2} \times S^{2}\right)\right)$ is given by $S_{1}, S_{2}, F_{1}, \ldots, F_{M}$, where $S_{1}:=\left[S^{2} \times\{\right.$ point $\left.\}\right], S_{2}:=\left[\{\right.$ point $\left.\} \times S^{2}\right]$ and $F_{1}, \ldots, F_{M}$ are the classes of the exceptional divisors.

Notice that there is a diffeomorphism $\varphi: X_{M}\left(S^{2} \times S^{2}\right) \rightarrow X_{M+1}\left(\mathbb{C} P^{2}\right)$ such that the induced map in homology is

$$
\begin{aligned}
\varphi_{*}: \quad H_{2}\left(X_{M}\left(S^{2} \times S^{2}\right)\right) & \longrightarrow & H_{2}\left(X_{M+1}\left(\mathbb{C} P^{2}\right)\right) \\
S_{1} & \longmapsto & L-E_{1} \\
S_{2} & \longmapsto & L \quad-E_{2} \\
F_{1} & \longmapsto & L-E_{1}-E_{2} \\
F_{i} & \longmapsto &
\end{aligned}
$$

The existence of such a $\varphi$ is clear from a moment map picture such as Figure 2.4 above. With respect to the new basis $S_{1}, S_{2}, F_{1}, \ldots, F_{M}$ we write an element of $H_{2}\left(X_{M}\left(S^{2} \times S^{2}\right)\right)$ as $\left(d, e ; m_{1}, \ldots, m_{M}\right)$. Then

$$
\varphi_{*}(d, e ; m)=\left(d+e-m_{1} ; d-m_{1}, e-m_{1}, m_{2}, \ldots, m_{M}\right) .
$$

In the new basis, the constraint given by a class in $\mathcal{E}$ can be written in a more useful form:

Proposition 3.8. $\quad$ (i) $\operatorname{All}(d, e ; m) \in \mathcal{E}_{M}$ satisfy the two Diophantine equations

$$
\begin{aligned}
\sum m_{i} & =2(d+e)-1, \\
\sum m_{i}^{2} & =2 d e+1 .
\end{aligned}
$$

(ii) For all distinct $(d, e ; m),\left(d^{\prime}, e^{\prime} ; m^{\prime}\right) \in \mathcal{E}_{M}$, we have

$$
\sum m_{i} m_{i}^{\prime} \leqslant d e^{\prime}+d^{\prime} e
$$

(iii) A tuple $(d, e ; m)$ belongs to $\mathcal{E}_{M}$ if and only if $(d, e ; m)$ satisfies the Diophantine equations of (i) and its image under $\varphi_{*}$ can be reduced to $(0 ;-1,0, \ldots, 0)$ by repeated Cremona moves.

Proof. Let $E \in \mathcal{E}$. The two identities in Proposition 3.6(i) correspond to $c_{1}(E)=1$ and $E \cdot E=-1$. For $E=d S_{1}+e S_{2}-\sum m_{i} F_{i}$ these identities 
become

$$
\begin{gathered}
c_{1}(E)=2 d+2 e-\sum m_{i}=1, \\
E \cdot E=-\sum m_{i}^{2}+2 d e=-1,
\end{gathered}
$$

proving (i). Assertion (ii) of Proposition 3.6 corresponds to positivity of intersection of $J$-holomorphic spheres representing $E, E^{\prime} \in \mathcal{E}$. For distinct elements $E=(d, e ; m)$ and $E^{\prime}=\left(d^{\prime}, e^{\prime} ; m^{\prime}\right)$ in $\mathcal{E}$ we thus have

$$
E \cdot E^{\prime}=d e^{\prime}+e d^{\prime}-\sum m_{i} m_{i}^{\prime} \geqslant 0
$$

proving (ii). Assertion (iii) holds since $\varphi_{*}$ is a base change.

In the sequel, given two vectors $m$ and $w$ of length $M$, we will denote by $\langle m, w\rangle=\sum_{i=1}^{M} m_{i} w_{i}$ the Euclidean scalar product in $\mathbb{R}^{M}$. Notice that we will also use this notation for vectors $m$ and $w$ of different lengths, meaning the Euclidean scalar product of the two vectors after adding enough zeros at the end of the shorter one.

Proposition 3.9. Let $a \geqslant 1$ be a rational number with weight expansion $w(a)=\left(w_{1}, \ldots, w_{M}\right)$. For $(d, e ; m) \in \mathcal{E}$, define the constraint

$$
\mu(d, e ; m)(a):=\frac{\langle m, w(a)\rangle}{d+e} .
$$

Then

$$
c(a)=\sup _{(d, e ; m) \in \mathcal{E}}\left\{\sqrt{\frac{a}{2}}, \mu(d, e ; m)(a)\right\} .
$$

Proof. By Lemma 3.1, $E(1, a) \stackrel{s}{\hookrightarrow} C(A)$ if and only if

$$
B(A) \sqcup B(A) \sqcup_{i} B\left(w_{i}\right) \stackrel{s}{\hookrightarrow} B(2 A) .
$$

By Theorem 3.2, this is true if and only if

$$
(2 A) l-A e_{1}-A e_{2}-\sum_{i=1}^{M} w_{i} e_{i+2} \in \mathcal{C}_{K}
$$


Denote by $s_{1}, s_{2}, f_{1}, \ldots, f_{M}$ the Poincaré duals of $S_{1}, S_{2}, F_{1}, \ldots, F_{M}$. The base change in cohomology is then

$$
\begin{aligned}
& \varphi^{*}: H^{2}\left(X_{M+1}\left(\mathbb{C} P^{2}\right)\right) \quad \longrightarrow \quad H^{2}\left(X_{M}\left(S^{2} \times S^{2}\right)\right) \\
& l \quad \longmapsto \quad s_{1}+s_{2}-f_{1} \\
& e_{1} \quad \longmapsto \quad s_{2}-f_{1} \\
& e_{2} \quad \longmapsto \quad s_{1} \quad-f_{1} \\
& e_{i} \quad \longmapsto \quad f_{i-1} \text {. }
\end{aligned}
$$

In this new basis of $H^{2}\left(X_{M}\left(S^{2} \times S^{2}\right)\right)$, 3.2 therefore becomes

$$
A s_{1}+A s_{2}-\sum_{i=1}^{M} w_{i} f_{i+1} \in \mathcal{C}_{K} .
$$

In view of Proposition 3.4. (3.3) translates to the conditions that for all $E:=$ $(d, e ; m) \in \mathcal{E}$, we have $2 A^{2}-\sum w_{i}^{2}>0$ and

$$
A s_{1}(E)+A s_{2}(E)-\sum w_{i} f_{i}(E)=(d+e) A-\sum m_{i} w_{i}>0 .
$$

Recall from Lemma 2.5 (ii) that $\sum w_{i}^{2}=a$. We conclude that $E(1, a) \stackrel{s}{\hookrightarrow} C(A)$ if and only if $A>\sqrt{\frac{a}{2}}$ and for all $(d, e ; m) \in \mathcal{E}$

$$
A>\frac{\sum m_{i} w_{i}}{d+e}=\frac{\langle m, w(a)\rangle}{d+e}
$$

This proves the proposition.

Remark 3.10. By the symmetry between $d$ and $e$ in the formula for $\mu(d, e$; $m)(a)$, we can assume that all elements $(d, e ; m) \in \mathcal{E}$ have $d \geqslant e$. We will use this convention throughout the paper.

The rest of this paper is devoted to the analysis of the constraints given in Proposition 3.9. This analysis follows the one in [MS. However, several modifications are necessary.

\section{Basic observations}

Lemma 4.1. For all $a \geqslant 8, c(a)=\sqrt{\frac{a}{2}}$. 
Proof. For all $(d, e ; m) \in \mathcal{E}$, we have by definition of $\mu$ and Proposition 3.8 (i)

$$
\mu(d, e ; m)(a):=\frac{\langle m, w(a)\rangle}{d+e} \leqslant \frac{\sum m_{i}}{d+e}=\frac{2(d+e)-1}{d+e}<2 \leqslant \sqrt{\frac{a}{2}}
$$

for all $a \geqslant 8$. Therefore, by Proposition $3.9, c(a)=\sqrt{\frac{a}{2}}$ for all $a \geqslant 8$.

Lemma 4.2. The function c has the following scaling property: for all $\lambda \geqslant 1$,

$$
\frac{c(\lambda a)}{\lambda a} \leqslant \frac{c(a)}{a}
$$

Proof. By definition of $c, E(1, a) \stackrel{s}{\hookrightarrow} C(c(a)+\varepsilon)$ for all $\varepsilon>0$. Since $E(1, a)$ symplectically embeds into $C(A)$ if and only if $E(\lambda, \lambda a)$ symplectically embeds into $C(\lambda A)$, this is equivalent to $E(\lambda, \lambda a) \stackrel{s}{\hookrightarrow} C(\lambda c(a)+\varepsilon)$ for all $\varepsilon>0$. Since $E(1, \lambda a) \subset E(\lambda, \lambda a)$ when $\lambda>1$, this implies that

$$
E(1, \lambda a) \stackrel{s}{\hookrightarrow} C(\lambda c(a)+\varepsilon)
$$

for all $\varepsilon>0$. Thus

$$
c(\lambda a):=\inf \{A: E(1, \lambda a) \stackrel{s}{\hookrightarrow} C(A)\} \leqslant \lambda c(a)=\lambda a \frac{c(a)}{a},
$$

as claimed.

Lemma 4.3. For $M \leqslant 7$, the sets $\mathcal{E}_{M}$ are finite and the only elements are

$$
\begin{array}{cccc}
(0,0 ;-1), & (1,0 ; 1), & \left(1,1 ; 1^{\times 3}\right), & \left(2,1 ; 1^{\times 5}\right), \\
\left(2,2 ; 2,1^{\times 5}\right), & \left(3,1 ; 1^{\times 7}\right), & \left(3,2 ; 2^{\times 2}, 1^{\times 5}\right), & \left(3,3 ; 2^{\times 4}, 1^{\times 3}\right), \\
\left(4,3 ; 2^{\times 6}, 1\right), & \left(4,4 ; 3,2^{\times 6}\right) . & &
\end{array}
$$

Proof. By Proposition 3.8(i), we have for a class $(d, e ; m) \in \mathcal{E}$ with $m=$ $\left(m_{1}, \ldots, m_{k}\right)$,

$$
(2(d+e)-1)^{2}=\left(\sum_{i=1}^{k} m_{i}\right)^{2} \leqslant k \sum_{i=1}^{k} m_{i}^{2}=k(2 d e+1),
$$

which is equivalent to

$$
4\left(d^{2}+e^{2}\right)+8 d e-4(d+e)+1 \leqslant 2 k d e+k
$$


and to

$$
d^{2}+e^{2} \leqslant \frac{k-4}{2} d e+d+e+\frac{k-1}{4} .
$$

Now, if $k \leqslant 7$, then

$$
d^{2}+e^{2} \leqslant \frac{3}{2} d e+d+e+\frac{3}{2} \leqslant \frac{3}{4}\left(d^{2}+e^{2}\right)+d+e+\frac{3}{2},
$$

using the fact that $2 d e \leqslant d^{2}+e^{2}$. This last inequality is equivalent to

$$
d^{2}-4 d+e^{2}-4 e \leqslant 6
$$

and finally to

$$
(d-2)^{2}+(e-2)^{2} \leqslant 14,
$$

which shows that $d, e \leqslant 5$. This shows that the sets $\mathcal{E}_{M}$ are finite for $M \leqslant 7$. To find the list of classes given above, it suffices to compute the solutions to the Diophantine equations of Proposition 3.8 (i) having $l(m) \leqslant 7$ and $d, e \leqslant 5$, and to show that they reduce to $(0,-1)$ by Cremona moves, which is the case.

Definition 4.4. A class $(d, e ; m) \in \mathcal{E}$ is said to be obstructive if there exists a rational number $a \geqslant 1$ such that $\mu(d, e ; m)(a)>\sqrt{\frac{a}{2}}$.

Lemma 4.5. Let $(d, e ; m) \in \mathcal{E}$ be an obstructive class. Then either $e=d$, or $e=d-1$.

Proof. Suppose by contradiction that there exists a class $(d, e ; m) \in \mathcal{E}$ obstructive at some point $a>1$ such that $d=e+k$ with $k \geqslant 2$. Then, using Proposition 3.8 (i) and Lemma 2.5(ii), we obtain

$$
\begin{aligned}
\sqrt{\frac{a}{2}} & <\mu(d, e ; m)(a)=\frac{\langle m, w(a)\rangle}{d+e} \leqslant \frac{\|m\|\|w(a)\|}{d+e}=\frac{\sqrt{2 d e+1} \sqrt{a}}{d+e} \\
& =\frac{\sqrt{2(e+k) e+1} \sqrt{a}}{2 e+k}=\frac{\sqrt{4 e^{2}+4 k e+2} \sqrt{a}}{\sqrt{2}(2 e+k)}<\frac{\sqrt{4 e^{2}+4 k e+k^{2}} \sqrt{a}}{\sqrt{2}(2 e+k)} \\
& =\sqrt{\frac{a}{2}},
\end{aligned}
$$

which is a contradiction.

Remark 4.6. This lemma will be very useful in the sequel, because whenever we will have to prove some properties of obstructive classes, it will be sufficient 
to prove them for classes of the form $(d, d ; m)$ or $\left(d+\frac{1}{2}, d-\frac{1}{2} ; m\right)$ only. Since this will happen many times, we will not explicitly refer to this lemma each time.

Definition 4.7. We define the error vector of a class $(d, e ; m)$ at a point $a$ as the vector $\varepsilon:=\varepsilon((d, e ; m), a)$ defined by the equation

$$
m=\frac{d+e}{\sqrt{2 a}} w(a)+\varepsilon .
$$

Lemma 4.8. Let $a=\frac{p}{q} \geqslant 1$ be a rational number with weight expansion $w(a)$ and let $(d, e ; m) \in \mathcal{E}$. Then

(i) $\mu(d, e ; m)(a) \leqslant \frac{\sqrt{2 d e+1} \sqrt{a}}{d+e}$. In particular,

$\mu(d, d ; m)(a) \leqslant \sqrt{1+\frac{1}{2 d^{2}}} \sqrt{\frac{a}{2}}$ and $\quad \mu\left(d+\frac{1}{2}, d-\frac{1}{2} ; m\right)(a) \leqslant \sqrt{1+\frac{1}{4 d^{2}}} \sqrt{\frac{a}{2}}$,

(ii) $\mu(d, e ; m)(a)>\sqrt{\frac{a}{2}}$ if and only if $\langle\varepsilon, w(a)\rangle>0$,

(iii) If $\mu(d, d ; m)(a)>\sqrt{\frac{a}{2}}\left(\right.$ resp. $\left.\mu\left(d+\frac{1}{2}, d-\frac{1}{2} ; m\right)(a)>\sqrt{\frac{a}{2}}\right)$, then $\langle\varepsilon, \varepsilon\rangle<$ 1 (resp. $\left.\langle\varepsilon, \varepsilon\rangle<\frac{1}{2}\right)$,

(iv) $-\sum_{i=1}^{M} \varepsilon_{i}=\frac{d+e}{\sqrt{2 a}}\left(y(a)-\frac{1}{q}\right)+1$, where $y(a):=a+1-2 \sqrt{2 a}$.

Proof. (i) By Cauchy-Schwarz inequality, Proposition 3.8(i) and Lemma 2.5 we have

$$
(d+e) \mu(d, e ; m)(a)=\langle m, w(a)\rangle \leqslant\|m\|\|w(a)\|=\sqrt{2 d e+1} \sqrt{a} .
$$

In the case of a class $(d, d ; m)$ we find that

$$
\mu(d, d ; m)(a) \leqslant \frac{\sqrt{2 d^{2}+1} \sqrt{a}}{2 d}=\sqrt{\frac{2 d^{2}+1}{2 d^{2}}} \sqrt{\frac{a}{2}}=\sqrt{1+\frac{1}{2 d^{2}}} \sqrt{\frac{a}{2}},
$$

and in the case of a class $\left(d+\frac{1}{2}, d-\frac{1}{2} ; m\right)$ that

$$
\mu\left(d+\frac{1}{2}, d-\frac{1}{2} ; m\right)(a) \leqslant \frac{\sqrt{2 d^{2}+\frac{1}{2}} \sqrt{a}}{2 d}=\sqrt{1+\frac{1}{4 d^{2}}} \sqrt{\frac{a}{2}} .
$$


(ii) Since

$$
\begin{aligned}
\langle\varepsilon, w(a)\rangle & =\left\langle m-\frac{d+e}{\sqrt{2 a}} w(a), w(a)\right\rangle=\langle m, w(a)\rangle-\frac{d+e}{\sqrt{2 a}}\|w(a)\|^{2} \\
& =\langle m, w(a)\rangle-(d+e) \sqrt{\frac{a}{2}}
\end{aligned}
$$

we see that $\langle\varepsilon, w(a)\rangle>0$ if and only if $\mu(d, e ; m)(a)=\frac{\langle m, w(a)\rangle}{d+e}>\sqrt{\frac{a}{2}}$.

(iii) For a class $(d, d ; m)$,

$$
\begin{aligned}
2 d^{2}+1 & =\langle m, m\rangle=\left\langle\sqrt{\frac{2}{a}} d w(a)+\varepsilon, \sqrt{\frac{2}{a}} d w(a)+\varepsilon\right\rangle \\
& =2 d^{2}+\frac{2 \sqrt{2}}{\sqrt{a}} d\langle w(a), \varepsilon\rangle+\langle\varepsilon, \varepsilon\rangle
\end{aligned}
$$

shows that if $\mu(d, d ; m)(a)>\sqrt{\frac{a}{2}}$, then by (ii) $\langle\varepsilon, \varepsilon\rangle<1$. Similarly, for a class $\left(d+\frac{1}{2}, d-\frac{1}{2} ; m\right)$,

$$
2 d^{2}+\frac{1}{2}=2 d^{2}+\frac{2 \sqrt{2}}{\sqrt{a}} d\langle w(a), \varepsilon\rangle+\langle\varepsilon, \varepsilon\rangle
$$

shows that if $\mu\left(d+\frac{1}{2}, d-\frac{1}{2} ; m\right)(a)>\sqrt{\frac{a}{2}}$, then $\langle\varepsilon, \varepsilon\rangle<\frac{1}{2}$.

(iv) By Proposition 3.8(i) and Lemma 2.5. we see that

$$
2(d+e)-1=\sum_{i=1}^{M} m_{i}=\frac{d+e}{\sqrt{2 a}}\left(a+1-\frac{1}{q}\right)+\sum_{i=1}^{M} \varepsilon_{i} .
$$

Thus

$$
-\sum_{i=1}^{M} \varepsilon_{i}=\frac{d+e}{\sqrt{2 a}}(a+1-2 \sqrt{2 a})-\frac{d+e}{q \sqrt{2 a}}+1,
$$

from which the result follows.

Corollary 4.9. Suppose that $c(a)>\sqrt{\frac{a}{2}}$ for some rational $a \geqslant 1$. Then

(i) There exist classes $(d, e ; m),\left(d^{\prime}, e^{\prime} ; m^{\prime}\right) \in \mathcal{E}$ (possibly equal) and $\varepsilon>0$ such that

$$
c(z)= \begin{cases}\mu(d, e ; m) & \text { if } z \in] a-\varepsilon, a], \\ \mu\left(d^{\prime}, e^{\prime} ; m^{\prime}\right) & \text { if } z \in[a, a+\varepsilon[.\end{cases}
$$


(ii) The set of classes $(d, e ; m) \in \mathcal{E}$ such that $\mu(d, e ; m)(a)=c(a)$ is finite.

(iii) For each of the intervals of (i), there exist rational coefficients $\alpha, \beta \geqslant 0$ such that $c(z)=\alpha+\beta z$.

Proof. Since $c(a)>\sqrt{\frac{a}{2}}$, there exists $D \in \mathbb{N}$ such that $c(a)>\sqrt{1+\frac{1}{D^{2}}} \sqrt{\frac{a}{2}}$.

Since $c$ is continuous, there exists $\varepsilon>0$ such that $c(z)>\sqrt{1+\frac{1}{D^{2}}} \sqrt{\frac{z}{2}}$ for all $z \in] a-\varepsilon, a+\varepsilon\left[\right.$. Now, if $\mu(d, e ; m)(z)>\sqrt{1+\frac{1}{D^{2}}} \sqrt{\frac{z}{2}}$, the inequalities of Lemma 4.8 (i) imply that $d \leqslant D$. There are thus only finitely many classes with $\mu(d, e ; m)(z)>\sqrt{1+\frac{1}{D^{2}}} \sqrt{\frac{z}{2}}$, and for all $\left.z \in\right] a-\varepsilon, a+\varepsilon[, c(z)$ is the supremum of $\mu(d, e ; m)(z)$ taken over finitely many classes. This proves (i) and (ii). To prove (iii), notice first that the constraints $\mu(d, e ; m)$ are piecewise linear functions. Indeed, let $w(a)=\left(w_{1}(a), w_{2}(a), \ldots\right)$ be the weight expansion of $a$, where the $w_{i}$ are seen as functions of $a$. Then the $w_{i}$ are piecewise linear functions and so is $\mu(d, e ; m)=\frac{\langle m, w\rangle}{d+e}$. We can thus write $c(z)=\alpha+\beta z$ for $z$ belonging to one of the intervals of (i). Now, since $c$ is nondecreasing, $\beta \geqslant 0$, and by the scaling property of Lemma 4.2 , $\alpha \geqslant 0$.

Definition 4.10. A class $(d, e ; m) \in \mathcal{E}$ is called perfect if there exists $b>1$ and $\kappa>0$ such that $m=\kappa w(b)$, that is, such that the vector $m$ is a multiple of the weight expansion of $b$.

Lemma 4.11. Let $(d, e ; m) \in \mathcal{E}$ be a perfect class for some $b>1$ with $d=e$ or $d=e+1$. Then $c(b)=\mu(d, e ; m)(b)>\sqrt{\frac{b}{2}}$ and $(d, e ; m)$ is the only class such that $\mu(d, e ; m)(b)=c(b)$.

Proof. We first treat the case $d=e$. Let $(d, d ; m) \in \mathcal{E}$ be a perfect class: $m=$ $\kappa w(b)$ for some $b>1$. By Proposition 3.8(i),

$$
2 d^{2}<2 d^{2}+1=\langle m, m\rangle=\kappa^{2}\langle w(b), w(b)\rangle=\kappa^{2} b
$$

from which we deduce that $d<\kappa \sqrt{\frac{b}{2}}$. Then

$$
\mu(d, d ; m)(b)=\frac{\langle m, w(b)\rangle}{2 d}=\frac{\kappa b}{2 d}>\sqrt{\frac{b}{2}} .
$$

This shows that $(d, d ; m)$ is obstructive at $b$. But then $(d, d ; m)$ is the only obstructive class at $b$. Indeed, if $\left(d^{\prime}, e^{\prime} ; m^{\prime}\right) \in \mathcal{E}$ is a class different of $(d, d ; m)$, 
by positivity of intersections (Proposition 3.8(ii)),

$$
\kappa\left\langle m^{\prime}, w(b)\right\rangle=\sum m_{i} m_{i}^{\prime} \leqslant d\left(d^{\prime}+e^{\prime}\right) .
$$

Thus

$$
\mu\left(d^{\prime}, e^{\prime} ; m^{\prime}\right)(b)=\frac{\left\langle m^{\prime}, w(b)\right\rangle}{d^{\prime}+e^{\prime}} \leqslant \frac{d\left\langle m^{\prime}, w(b)\right\rangle}{\kappa\left\langle m^{\prime}, w(b)\right\rangle}=\frac{d}{\kappa}<\sqrt{\frac{b}{2}} .
$$

Consider now a class of the form $\left(d+\frac{1}{2}, d-\frac{1}{2} ; m\right)$. By Proposition 3.8 (i), we have

$$
2 d^{2}\left\langle 2 d^{2}+\frac{1}{2}=\langle m, m\rangle=\kappa^{2}\langle w(b), w(b)\rangle=\kappa^{2} b,\right.
$$

and thus $d<\kappa \sqrt{\frac{b}{2}}$ as in the case of a class $(d, d ; m)$. The rest of the proof is then identical.

Definition 4.12. Define the length of a vector $m$, denoted by $l(m)$, as the number of positive entries in $m$, and denote by $l(a)$ the length of the weight expansion $w(a)$ of $a$.

Lemma 4.13. Let $(d, e ; m) \in \mathcal{E}$ be an obstructive class. Let I be a maximal nonempty open interval on which $\mu(d, e ; m)(a)>\sqrt{\frac{a}{2}}$. Then there exists a unique $a_{0} \in I$ such that $l\left(a_{0}\right)=l(m)$. Moreover for all $a \in I, l(a) \geqslant l\left(a_{0}\right)$.

Proof. Let us first prove that for all $a \in I, l(a) \geqslant l(m)$. If $l(a)<l(m)$, then, by Proposition 3.8(i),

$$
\sum_{i=1}^{l(a)} m_{i}^{2}<2 d e+1
$$

Thus

$$
\mu(d, e ; m)(a)=\frac{\langle m, w(a)\rangle}{d+e} \leqslant \frac{\sqrt{\sum_{i=1}^{l(a)} m_{i}^{2}}\|w(a)\|}{d+e} \leqslant \frac{\sqrt{4 d e}}{d+e} \sqrt{\frac{a}{2}} \leqslant \sqrt{\frac{a}{2}},
$$

and so $a \notin I$. Let us now prove the existence of an $a_{0}$ with $l\left(a_{0}\right)=l(m)$. Let $w(a)=\left(w_{1}(a), w_{2}(a), \ldots\right)$ be the weight expansion of $a$, where the $w_{i}$ are again seen as functions of $a$. The $w_{i}$ are piecewise linear functions and are linear on intervals that do not contain elements $a^{\prime}$ with $l\left(a^{\prime}\right) \leqslant i$. Hence if all $a \in I$ would have $l(a)>l(m)$, then the $l(m)$ first $w_{i}$ would be linear, and $\mu(d, e ; m)$ also. But this is impossible since $\sqrt{\frac{a}{2}}$ is concave. Thus there exists $a_{0} \in I$ with $l\left(a_{0}\right)=l(m)$. The proof of uniqueness of $a_{0}$ follows from the fact that if $a<b$ and $l(a)=l(b)$, then there exists $c \in] a, b[$ such that $l(c)<l(a)$. 
Lemma 4.14. Let $(d, e ; m) \in \mathcal{E}$ be such that $\mu(d, e ; m)(a)>\sqrt{\frac{a}{2}}$. Let $J=$ $\{k, \ldots, k+s-1\}$ be a block of $s \geqslant 2$ consecutive integers such that the $w_{i}(a)$ are equal for all $i \in J$. Then we have the three following possibilities

$$
\begin{array}{ll}
\text { 1. } & m_{k}=\cdots=m_{k+s-1} \\
\text { 2. } & m_{k}-1=m_{k+1}=\cdots=m_{k+s-1} \\
\text { 3. } & m_{k}=\cdots=m_{k+s-2}=m_{k+s-1}+1 .
\end{array}
$$

Moreover, there is at most one block of length $s \geqslant 2$ where the $m_{i}$ are not all equal, and if such a block $J$ exists, then $\sum_{i \in J} \varepsilon_{i}^{2} \geqslant \frac{s-1}{s}$.

The proof is similar to the proof of Lemma 2.1.7 in [MS].

Corollary 4.15. If a class of the form $\left(d+\frac{1}{2}, d-\frac{1}{2} ; m\right)$ is obstructive, then the $m_{i}$ are constant on each block.

Proof. Suppose there exists a block $J$ of length $s \geqslant 2$ on which the $w_{i}(a)$ are not all equal. Then by Lemma $4.14 \sum_{i \in J} \varepsilon_{i}^{2} \geqslant \frac{s-1}{s} \geqslant \frac{1}{2}$, which contradicts Lemma 4.8 (iii), which states that $\sum \varepsilon_{i}^{2}<\frac{1}{2}$.

Lemma 4.16. Let $(d, e ; m) \in \mathcal{E}$ be an obstructive class at some rational $a \geqslant$ 1 with $l(a)=l(m)$. Let $w_{k+1}, \ldots, w_{k+s}$ be a block which is not the first block of $w(a)$.

(i) If the block is not the last one, then

$$
\left|m_{k}-\left(m_{k+1}+\cdots+m_{k+s+1}\right)\right|<\sqrt{s+2}
$$

If the block is the last one, then

$$
\left|m_{k}-\left(m_{k+1}+\cdots+m_{k+s}\right)\right|<\sqrt{s+1}
$$

(ii) It is always the case that

$$
m_{k}-\sum_{i=k+1}^{M} m_{i}<\sqrt{M-k+1}
$$

The proof is similar to the one of Lemma 2.1.8 in [MS].

Proposition 4.17. Let $(d, e ; m) \in \mathcal{E}$ be an obstructive class at a point $a=$ : $\frac{p}{q} \in \mathbb{Q}$ written in lowest terms with $l(a)=l(m)$. Let $m_{M}$ be the last nonzero entry of the vector $m$ and let $I$ be the maximal open interval containing a such 
that $\mu(d, e ; m)(a)>\sqrt{\frac{a}{2}}$. Then there exist integers $A<p$ and $B<\left(m_{M}+1\right) q$ such that

$$
(d+e) \mu(d, e ; m)(z)= \begin{cases}A+B z & \text { if } z \leqslant a, z \in I \\ \left(A+m_{M} p\right)+\left(B-m_{M} q\right) z & \text { if } z \geqslant a, z \in I\end{cases}
$$

Again, the proof is similar to the one of Proposition 2.3.2 in [MS].

\section{The interval $\left[1, \sigma^{2}\right]$}

The goal of this section is to prove part (i) of Theorem 1.3 .

\subsection{Preliminaries}

Let us first recall that the Pell numbers $P_{n}$ and the half companion Pell numbers $H_{n}$ are defined by the recurrence relations

$$
\begin{aligned}
& P_{0}=0, \quad P_{1}=1, \quad P_{n}=2 P_{n-1}+P_{n-2} \\
& H_{0}=1, \quad H_{1}=1, \quad H_{n}=2 H_{n-1}+H_{n-2}
\end{aligned}
$$

respectively. It is then easy to see that

$$
H_{n}=P_{n}+P_{n-1}
$$

Using this, we define the sequence $\left(\alpha_{n}\right)_{n \geqslant 0}$ by

$$
\alpha_{n}:= \begin{cases}\frac{2 P_{n+1}^{2}}{H_{n}^{2}}=: \frac{p_{n}}{q_{n}} & \text { if } n \text { is even } \\ \frac{H_{n+1}^{2}}{2 P_{n}^{2}}=: \frac{p_{n}}{q_{n}} & \text { if } n \text { is odd }\end{cases}
$$

Set $W\left(\alpha_{n}\right)=q_{n} w\left(\alpha_{n}\right)$. Then, define $W^{\prime}\left(\alpha_{n}\right)$ as the tuple obtained from $W\left(\alpha_{n}\right)$ by adding an extra 1 at the end. Define the classes $E\left(\alpha_{n}\right)$ by

$$
E\left(\alpha_{n}\right):= \begin{cases}\left(P_{n+1} H_{n}, P_{n+1} H_{n} ; W^{\prime}\left(\alpha_{n}\right)\right) & \text { if } n \text { is even } \\ \left(P_{n} H_{n+1}, P_{n} H_{n+1} ; W^{\prime}\left(\alpha_{n}\right)\right) & \text { if } n \text { is odd }\end{cases}
$$


For instance,

$$
\begin{aligned}
& E\left(\alpha_{0}\right)=\left(1,1 ; 1^{\times 3}\right), \\
& E\left(\alpha_{1}\right)=\left(3,3 ; 2^{\times 4}, 1^{\times 3}\right), \\
& E\left(\alpha_{2}\right)=\left(15,15 ; 9^{\times 5}, 5,4,1^{\times 5}\right), \\
& E\left(\alpha_{3}\right)=\left(85,85 ; 50^{\times 5}, 39,11^{\times 3}, 6,5,1^{\times 6}\right) .
\end{aligned}
$$

Moreover, we define the sequence $\left(\beta_{n}\right)_{n \geqslant 0}$ by

$$
\beta_{n}:= \begin{cases}\frac{H_{n+2}}{H_{n}}=: \frac{p_{n}}{q_{n}} & \text { if } n \text { is even, } \\ \frac{P_{n+2}}{P_{n}}=: \frac{p_{n}}{q_{n}} & \text { if } n \text { is odd } .\end{cases}
$$

Set $W\left(\beta_{n}\right)=q_{n} w\left(\beta_{n}\right)$. Then the classes $E\left(\beta_{n}\right)$ are defined by

$$
E\left(\beta_{n}\right):= \begin{cases}\left(\frac{1}{4}\left(H_{n}+H_{n+2}\right), \frac{1}{4}\left(H_{n}+H_{n+2}\right) ; W\left(\beta_{n}\right)\right) & \text { if } n \text { is even, } \\ \left.\frac{1}{4}\left(P_{n}+P_{n+2}\right)+\frac{1}{2}, \frac{1}{4}\left(P_{n}+P_{n+2}\right)-\frac{1}{2} ; W\left(\beta_{n}\right)\right) & \text { if } n \text { is odd. }\end{cases}
$$

For instance,

$$
\begin{aligned}
& E\left(\beta_{0}\right)=\left(1,1 ; 1^{\times 3}\right), \\
& E\left(\beta_{1}\right)=\left(2,1 ; 1^{\times 5}\right), \\
& E\left(\beta_{2}\right)=\left(5,5 ; 3^{\times 5}, 2,1^{\times 2}\right), \\
& E\left(\beta_{3}\right)=\left(9,8 ; 5^{\times 5}, 4,1^{\times 4}\right) .
\end{aligned}
$$

Theorem 5.1. For all $n \geqslant 0, E\left(\alpha_{n}\right), E\left(\beta_{n}\right) \in \mathcal{E}$.

The proof that $E\left(\alpha_{n}\right) \in \mathcal{E}$ is given in the next subsection, while the proof that $E\left(\beta_{n}\right) \in \mathcal{E}$ is given in Corollary 6.19. Theorem 5.1 implies part (i) of Theorem 1.3 .

Corollary 5.2. On the interval $\left[1, \sigma^{2}\right]$,

$$
c(a)=\left\{\begin{array}{cl}
1 & \text { if } a \in[1,2], \\
\frac{1}{\sqrt{2 \alpha_{n}}} a & \text { if } a \in\left[\alpha_{n}, \beta_{n}\right], \\
\sqrt{\frac{\alpha_{n+1}}{2}} & \text { if } a \in\left[\beta_{n}, \alpha_{n+1}\right],
\end{array}\right.
$$

for all $n \geqslant 0$. 
Proof. Since for all $n \geqslant 0, E\left(\beta_{n}\right)$ is a perfect class, we know by Lemma 4.11 that $c\left(\beta_{n}\right)=\mu\left(E\left(\beta_{n}\right)\right)\left(\beta_{n}\right)$. Hence

$$
c\left(\beta_{n}\right)=\sqrt{\frac{\alpha_{n+1}}{2}}
$$

for all $n \geqslant 0$. Indeed, for $n$ even, we compute

$$
\begin{aligned}
c\left(\beta_{n}\right) & =\frac{2 H_{n}\left\langle w\left(\beta_{n}\right), w\left(\beta_{n}\right)\right\rangle}{H_{n+2}+H_{n}}=\frac{2 H_{n} \beta_{n}}{H_{n+2}+H_{n}}=\frac{2 H_{n+2}}{H_{n+2}+H_{n}} \\
& =\frac{2\left(P_{n+2}+P_{n+1}\right)}{P_{n+2}+P_{n+1}+P_{n}+P_{n-1}}=\frac{2\left(P_{n+2}+P_{n+1}\right)}{4 P_{n+1}}=\frac{H_{n+2}}{2 P_{n+1}} \\
& =\sqrt{\frac{\alpha_{n+1}}{2}}
\end{aligned}
$$

and for $n$ odd,

$$
\begin{aligned}
c\left(\beta_{n}\right) & =\frac{2 P_{n}\left\langle w\left(\beta_{n}\right), w\left(\beta_{n}\right)\right\rangle}{P_{n+2}+P_{n}}=\frac{2 P_{n} \beta_{n}}{P_{n+2}+P_{n}}=\frac{2 P_{n+2}}{P_{n+2}+P_{n}} \\
& =\frac{P_{n+2}}{\frac{1}{2}\left(P_{n+2}-P_{n}\right)+P_{n}}=\frac{P_{n+2}}{P_{n+1}+P_{n}}=\frac{P_{n+2}}{H_{n}}=\sqrt{\frac{\alpha_{n+1}}{2} .}
\end{aligned}
$$

Furthermore, $c\left(\alpha_{n}\right)=\sqrt{\frac{\alpha_{n}}{2}}$ for all $n \geqslant 0$. Indeed, for $n$ even, we have

$$
\mu\left(E\left(\alpha_{n}\right)\right)\left(\alpha_{n}\right)=\frac{H_{n}^{2} \alpha_{n}}{2 P_{n+1} H_{n}}=\frac{H_{n} \alpha_{n}}{2 P_{n+1}}=\frac{P_{n+1}}{H_{n}}=\sqrt{\frac{\alpha_{n}}{2}} .
$$

Thus for all $(d, e ; m) \in \mathcal{E}$ distinct from $E\left(\alpha_{n}\right)$, we get by Proposition 3.8 (ii) that

$$
P_{n+1} H_{n}(d+e) \geqslant\left\langle m, W^{\prime}\left(\alpha_{n}\right)\right\rangle \geqslant H_{n}^{2}\left\langle m, w\left(\alpha_{n}\right)\right\rangle,
$$

and hence

$$
\mu(d, e ; m)\left(\alpha_{n}\right)=\frac{\left\langle m, w\left(\alpha_{n}\right)\right\rangle}{d+e} \leqslant \frac{P_{n+1} H_{n}}{H_{n}^{2}}=\frac{P_{n+1}}{H_{n}}=\sqrt{\frac{\alpha_{n}}{2}} .
$$

Next, for $n$ odd, we have

$$
\mu\left(E\left(\alpha_{n}\right)\right)\left(\alpha_{n}\right)=\frac{2 P_{n}^{2} \alpha_{n}}{2 P_{n} H_{n+1}}=\frac{P_{n} \alpha_{n}}{H_{n+1}}=\frac{H_{n+1}}{2 P_{n}}=\sqrt{\frac{\alpha_{n}}{2}} .
$$

Thus for all $(d, e ; m) \in \mathcal{E}$ distinct from $E\left(\alpha_{n}\right)$, we get

$$
P_{n} H_{n+1}(d+e) \geqslant\left\langle m, W^{\prime}\left(\alpha_{n}\right)\right\rangle \geqslant 2 P_{n}^{2}\left\langle m, w\left(\alpha_{n}\right)\right\rangle,
$$


and hence

$$
\mu(d, e ; m)\left(\alpha_{n}\right)=\frac{\left\langle m, w\left(\alpha_{n}\right)\right\rangle}{d+e} \leqslant \frac{P_{n} H_{n+1}}{2 P_{n}^{2}}=\frac{H_{n+1}}{2 P_{n}}=\sqrt{\frac{\alpha_{n}}{2}} .
$$

Thus, by Proposition 3.9 we have $c\left(\alpha_{n}\right)=\sqrt{\frac{\alpha_{n}}{2}}$ for all $n \geqslant 0$ as required. Since $c$ is nondecreasing, we get that $c(a)=\sqrt{\frac{\alpha_{n+1}}{2}}$ for $a \in\left[\beta_{n}, \alpha_{n+1}\right]$. Moreover, we have that for all $n \geqslant 0$

$$
\frac{c\left(\beta_{n}\right)}{\beta_{n}}=\frac{c\left(\alpha_{n}\right)}{\alpha_{n}}
$$

Indeed, for $n$ even,

$$
\frac{c\left(\beta_{n}\right)}{\beta_{n}}=\frac{\sqrt{\frac{\alpha_{n+1}}{2}}}{\beta_{n}}=\frac{H_{n}}{2 P_{n+1}}=\frac{\sqrt{\frac{\alpha_{n}}{2}}}{\alpha_{n}}=\frac{c\left(\alpha_{n}\right)}{\alpha_{n}},
$$

and for $n$ odd,

$$
\frac{c\left(\beta_{n}\right)}{\beta_{n}}=\frac{\sqrt{\frac{\alpha_{n+1}}{2}}}{\beta_{n}}=\frac{P_{n}}{H_{n+1}}=\frac{\sqrt{\frac{\alpha_{n}}{2}}}{\alpha_{n}}=\frac{c\left(\alpha_{n}\right)}{\alpha_{n}} .
$$

Hence, by the scaling property of Lemma 4.2, the function $c$ has to be linear on $\left[\alpha_{n}, \beta_{n}\right]$ and thus $c(a)=\frac{1}{\sqrt{2 \alpha_{n}}} a$ for $a \in\left[\alpha_{n}, \beta_{n}\right]$.

\subsection{The classes $E\left(\alpha_{n}\right)$ belong to $\mathcal{E}$}

Lemma 5.3. The classes $E\left(\alpha_{n}\right)$ satisfy the Diophantine conditions of Proposition $3.8(i)$.

Proof. We will prove this separately for $n$ even and odd. In both cases, we will use Lemma 2.5 and the relation

$$
-P_{2 m}^{2}+2 P_{2 m} P_{2 m-1}+P_{2 m-1}^{2}=1
$$

which can be easily deduced from the following identity

$$
P_{2 m-k}=(-1)^{k+1}\left(P_{k} H_{2 m}-H_{k} P_{2 m}\right),
$$


given in Corollary $6.8(\mathrm{v})$. For $n=2 m$, we obtain

$$
\begin{aligned}
\sum m_{i} & =H_{2 m}^{2} \sum w_{i}+1=H_{2 m}^{2}\left(\frac{2 P_{2 m+1}^{2}}{H_{2 m}^{2}}+1-\frac{1}{H_{2 m}^{2}}\right)+1 \\
& =2\left(2 P_{2 m}+P_{2 m-1}\right)^{2}+\left(P_{2 m}+P_{2 m-1}\right)^{2} \\
& =9 P_{2 m}^{2}+10 P_{2 m} P_{2 m-1}+3 P_{2 m-1}^{2} \\
& =8 P_{2 m}^{2}+12 P_{2 m} P_{2 m-1}+4 P_{2 m-1}^{2}-\left(-P_{2 m}^{2}+2 P_{2 m} P_{2 m-1}+P_{2 m-1}^{2}\right) \\
& =4 P_{2 m+1} H_{2 m}-1=2(d+e)-1 ; \\
\sum m_{i}^{2} & =H_{2 m}^{4} \sum w_{i}^{2}+1=H_{2 m}^{4} \frac{2 P_{2 m+1}^{2}}{H_{2 m}^{2}}+1 \\
& =2 P_{2 m+1}^{2} H_{2 m}^{2}+1=2 d e+1 .
\end{aligned}
$$

Moreover, for $n=2 m-1$,

$$
\begin{aligned}
\sum m_{i} & =2 P_{2 m-1}^{2} \sum w_{i}+1=2 P_{2 m-1}^{2}\left(\frac{H_{2 m}^{2}}{2 P_{2 m-1}^{2}}+1-\frac{1}{2 P_{2 m-1}^{2}}\right)+1 \\
& =\left(P_{2 m}+P_{2 m-1}\right)^{2}+2 P_{2 m-1}^{2} \\
& =P_{2 m}^{2}+2 P_{2 m} P_{2 m-1}+3 P_{2 m-1}^{2} \\
& =4 P_{2 m} P_{2 m-1}+4 P_{2 m-1}^{2}-\left(-P_{2 m}^{2}+2 P_{2 m} P_{2 m-1}+P_{2 m-1}^{2}\right) \\
& =4 P_{2 m-1} H_{2 m}-1=2(d+e)-1 ; \\
\sum m_{i}^{2} & =4 P_{2 m-1}^{4} \sum w_{i}^{2}+1=4 P_{2 m-1}^{4} \frac{H_{2 m}^{2}}{2 P_{2 m-1}^{2}}+1 \\
& =2 P_{2 m-1}^{2} H_{2 m}^{2}+1=2 d e+1 .
\end{aligned}
$$

This proves the lemma.

We will now prove separately for $n$ even and $n$ odd that the classes $E\left(\alpha_{n}\right)$ reduce to $(0 ;-1)$ by standard Cremona moves.

5.2.1. The classes $\boldsymbol{E}\left(\alpha_{2 m}\right)$ reduce to $(0 ;-1)$. One readily checks that the classes $E\left(\alpha_{2 m}\right)$ reduce to $(0 ;-1)$ for $m=0,1,2$. In the following, we reduce the classes $E\left(\alpha_{2 m}\right)$ for $m \geqslant 3$.

Lemma 5.4. The continued fraction expansion of $\alpha_{2 m}$ is

$$
\left[5 ;\{1,4\}^{\times(m-1)}, 1,1,3,1,\{4,1\}^{\times(m-1)}\right] .
$$


Moreover, with $u_{j}:=\left(2 H_{j}-P_{j}\right) H_{2 m}+H_{j} P_{2 m}$,

$$
\begin{aligned}
E\left(\alpha_{2 m}\right)= & \left(P_{2 m+1} H_{2 m}, P_{2 m+1} H_{2 m} ;\right. \\
& \left(\frac{1}{2} u_{2 m}\right)^{\times 5}, u_{2 m-1},\left(\frac{1}{2} u_{2 m-2}\right)^{\times 4}, \ldots, u_{3},\left(\frac{1}{2} u_{2}\right)^{\times 4}, \\
& u_{1}, H_{2 m}+\frac{1}{2} P_{2 m},\left(\frac{1}{2} P_{2 m}\right)^{\times 3}, P_{2 m-1}, \\
& \left.\left(\frac{1}{2} P_{2 m-2}\right)^{\times 4}, P_{2 m-3}, \ldots,\left(\frac{1}{2} P_{2}\right)^{\times 4}, P_{1}, 1\right) .
\end{aligned}
$$

Proof. Since $\left(\alpha_{n}\right)$ is an increasing sequence converging to $\sigma^{2}<6$ and $\alpha_{2}=$ $\frac{50}{9}>5$, the first term of $W^{\prime}\left(\alpha_{2 m}\right)$ is

$$
\left(q_{2 m}\right)^{\times 5}=\left(H_{2 m}^{2}\right)^{\times 5}=\left(\frac{1}{2} u_{2 m}\right)^{\times 5} .
$$

To determine the next terms, we will first prove that for all $k \geqslant 1, u_{2 k+1}>$ $\frac{1}{2} u_{2 k}>u_{2 k-1}$. Indeed

$$
\begin{aligned}
u_{2 k+1} & =\left(2 H_{2 k+1}-P_{2 k+1}\right) H_{2 m}+H_{2 k+1} P_{2 m} \\
& =\left(4 P_{2 k}+P_{2 k-1}\right) H_{2 m}+\left(2 H_{2 k}+H_{2 k-1}\right) P_{2 m} \\
& >\left(\frac{1}{2} P_{2 k}+P_{2 k-1}\right) H_{2 m}+\frac{1}{2} H_{2 k} P_{2 m} \\
& =\frac{1}{2}\left(2 H_{2 k}-P_{2 k}\right) H_{2 m}+\frac{1}{2} H_{2 k} P_{2 m} \\
& =\frac{1}{2} u_{2 k} \\
& =\left(P_{2 k-1}+\frac{5}{2} P_{2 k-2}+P_{2 k-3}\right) H_{2 m}+\left(H_{2 k-1}+\frac{1}{2} H_{2 k-2}\right) P_{2 m} \\
& >\left(P_{2 k-1}+2 P_{2 k-2}\right) H_{2 m}+H_{2 k-1} P_{2 m} \\
& =\left(2 H_{2 k-1}-P_{2 k-1}\right) H_{2 m}+H_{2 k-1} P_{2 m} \\
& =u_{2 k-1} .
\end{aligned}
$$

The second term of $W^{\prime}\left(\alpha_{2 m}\right)$ is

$$
\begin{aligned}
2 P_{2 m+1}^{2}-5 H_{2 m}^{2} & =3 P_{2 m}^{2}-2 P_{2 m} P_{2 m-1}-3 P_{2 m-1}^{2} \\
& =\left(2 H_{2 m-1}-P_{2 m-1}\right) H_{2 m}+H_{2 m-1} P_{2 m} \\
& =u_{2 m-1}<\frac{1}{2} u_{2 m} .
\end{aligned}
$$


Now for all $k \geqslant 1$, we have

$$
\begin{aligned}
u_{2 k+1}-4\left(\frac{1}{2} u_{2 k}\right)= & \left(2 H_{2 k+1}-P_{2 k+1}-4 H_{2 k}+2 P_{2 k}\right) H_{2 m} \\
& +\left(H_{2 k+1}-2 H_{2 k}\right) P_{2 m} \\
= & \left(2 H_{2 k-1}-P_{2 k-1}\right) H_{2 m}+H_{2 k-1} P_{2 m}=u_{2 k-1}<\frac{1}{2} u_{2 k} \\
\frac{1}{2} u_{2 k}-u_{2 k-1}= & \left(H_{2 k}-\frac{1}{2} P_{2 k}-2 H_{2 k-1}+P_{2 k-1}\right) H_{2 m} \\
& +\left(\frac{1}{2} H_{2 k}-H_{2 k-1}\right) P_{2 m} \\
= & \frac{1}{2}\left(2 H_{2 k-2}-P_{2 k-2}\right) H_{2 m}+\frac{1}{2} H_{2 k-2} P_{2 m}=\frac{1}{2} u_{2 k-2} \\
< & u_{2 k-1} .
\end{aligned}
$$

Thus the first terms of $W^{\prime}\left(\alpha_{2 m}\right)$ are

$$
\left(\frac{1}{2} u_{2 m}\right)^{\times 5}, u_{2 m-1},\left(\frac{1}{2} u_{2 m-2}\right)^{\times 4}, \ldots, u_{3},\left(\frac{1}{2} u_{2}\right)^{\times 4}, u_{1} \text {. }
$$

The next terms are $H_{2 m}+\frac{1}{2} P_{2 m},\left(\frac{1}{2} P_{2 m}\right)^{\times 3}, P_{2 m-1}$. Indeed

$$
\begin{aligned}
\frac{1}{2} u_{2}-u_{1} & =H_{2 m}+\frac{1}{2} P_{2 m}<H_{2 m}+P_{2 m}=u_{1} ; \\
u_{1}-\left(H_{2 m}+\frac{1}{2} P_{2 m}\right) & =\frac{1}{2} P_{2 m}<H_{2 m}+\frac{1}{2} P_{2 m} ; \\
H_{2 m}+\frac{1}{2} P_{2 m}-3\left(\frac{1}{2} P_{2 m}\right) & =P_{2 m-1}<\frac{1}{2} P_{2 m} .
\end{aligned}
$$

Notice that for all $k \geqslant 1$,

$$
P_{k+1}>\frac{1}{2} P_{k}>P_{k-1}
$$

and thus

$$
P_{2 k+1}-4\left(\frac{1}{2} P_{2 k}\right)=P_{2 k-1}<\frac{1}{2} P_{2 k} ; \quad \frac{1}{2} P_{2 k}-P_{2 k-1}=\frac{1}{2} P_{2 k-2}<P_{2 k-1} .
$$

This proves that the last terms of $W^{\prime}\left(\alpha_{2 m}\right)$ are

$$
\left(\frac{1}{2} P_{2 m-2}\right)^{\times 4}, P_{2 m-3}, \ldots,\left(\frac{1}{2} P_{2}\right)^{\times 4}, P_{1}, 1
$$

with the last 1 added by definition of $W^{\prime}\left(\alpha_{2 m}\right)$. 
Let us introduce now some notations in order to simplify the expressions of the classes.

Definition 5.5. Set

$$
\begin{aligned}
A_{k}^{m}:= & \left(\left(\frac{1}{2} u_{2 k}\right)^{\times 4}, u_{2 k-1},\left(P_{2 k} H_{2 m}\right)^{\times 2},\left(\frac{1}{2} u_{2 k-2}\right)^{\times 4}, u_{2 k-3}, \ldots,\right. \\
& \left.\left(\frac{1}{2} u_{2}\right)^{\times 4}, u_{1}, H_{2 m}+\frac{1}{2} P_{2 m},\left(\frac{1}{2} P_{2 m}\right)^{\times 3}, P_{2 m-1}\right), \\
B_{k}^{m}:= & \left(\left(\frac{1}{2} P_{2 m-2}\right)^{\times 4}, P_{2 m-3}, \ldots,\left(\frac{1}{2} P_{2 m-2 k+2}\right)^{\times 4}, P_{2 m-2 k+1},\right. \\
& \left.\left(\frac{1}{2} P_{2 m-2 k}\right)^{\times 8},\left(P_{2 m-2 k-1}\right)^{\times 2}, \ldots,\left(\frac{1}{2} P_{2}\right)^{\times 8},\left(P_{1}\right)^{\times 2}, 1\right), \\
V_{k}^{m}:= & \left(P_{2 k+1} H_{2 m}+H_{2 k} P_{2 m} ; A_{k}^{m}, B_{k}^{m}\right) .
\end{aligned}
$$

Thus $A_{k}^{m}$ has the structure $\left[4,1,2,\{4,1\}^{\times(k-1)}, 1,3,1\right]$ and $B_{k}^{m}$ has the structure $\left[\{4,1\}^{\times(k-1)},\{8,2\}^{\times(m-k)}, 1\right]$. We use here the convention that if $k=m$, $B_{m}^{m}$ has the structure $\left[\{4,1\}^{\times(m-1)}, 1\right]$ and that if $k=1, B_{1}^{m}$ has the structure $\left[\{8,2\}^{\times(m-1)}, 1\right]$.

The structure of the reduction process of a class $E\left(\alpha_{2 m}\right)$ will be the following. First, we compute in Lemma 5.7 that the image of $E\left(\alpha_{2 m}\right)$ under $\varphi_{*}$ is $V_{m}^{m}=\left(P_{2 m+1} H_{2 m}+H_{2 m} P_{2 m} ; A_{m}^{m}, B_{m}^{m}\right)$. Then, we reduce $V_{m}^{m}$ in Lemma 5.8 and Lemma 5.9 to $V^{m}:=\left(H_{2 m} ; H_{2 m}-\frac{1}{2} P_{2 m},\left(\frac{1}{2} P_{2 m}\right)^{\times 3},\left(P_{2 m-1}\right)^{\times 2}, B_{1}^{m}\right)$ in $4(m-2)+8$ Cremona moves. Finally, we show in Lemmas 5.10 and 5.11 that $V^{m}$ reduces to $(0 ;-1)$ in $5(m-2)+8$ moves.

Remark 5.6. Since the Cremona transform of a class $(d ; m)$ only modifies the first 3 entries of the vector $m$, we will write some of the first entries of the classes and will abbreviate the other terms by $(*)$. It is important to notice that the terms denoted by $(*)$ will always be left invariant during the reduction process. Then, each time after applying the Cremona transform, we will reorder the entries of $m$. We will not always reorder the entries in decreasing order, but this will have no consequence on the reduction because we will reorder them in a way such that the first 3 entries of the vector $m$ will always be the 3 biggest entries in decreasing order. We will write down each step of the reduction, that is the class obtained after applying the Cremona transform and reordering. But sometimes when the reordering will not be 
obvious, we will write the intermediate step before reordering and denote by $\rightarrow$ the Cremona transform of a class and by $\rightsquigarrow$ the reordering of a class. We will freely use the three relations

$$
\begin{aligned}
P_{n} & =2 P_{n-1}+P_{n-2}, \quad H_{n}=P_{n}+P_{n-1}, \\
P_{2 m-k} & =(-1)^{k+1}\left(P_{k} H_{2 m}-H_{k} P_{2 m}\right) .
\end{aligned}
$$

Lemma 5.7. The image of $E\left(\alpha_{2 m}\right)$ by $\varphi_{*}$ is the class

$$
\varphi_{*}\left(E\left(\alpha_{2 m}\right)\right)=\left(P_{2 m+1} H_{2 m}+H_{2 m} P_{2 m} ; A_{m}^{m}, B_{m}^{m}\right)=V_{m}^{m} .
$$

Proof. The first terms of $E\left(\alpha_{2 m}\right)$ are $\left(P_{2 m+1} H_{2 m}, P_{2 m+1} H_{2 m} ; \frac{1}{2} u_{2 m},(*)\right)$. Since $\frac{1}{2} u_{2 m}=H_{2 m}^{2}$, we get

$$
\begin{aligned}
\varphi_{*}\left(E\left(\alpha_{2 m}\right)\right) & =\left(2 P_{2 m+1} H_{2 m}-H_{2 m}^{2} ;\left(P_{2 m+1} H_{2 m}-H_{2 m}^{2}\right)^{\times 2},(*)\right) \\
& =\left(P_{2 m+1} H_{2 m}+H_{2 m} P_{2 m} ;\left(P_{2 m} H_{2 m}\right)^{\times 2},(*)\right) .
\end{aligned}
$$

After reordering this class, we see that we indeed obtain $V_{m}^{m}$ as required.

Lemma 5.8. For all $3 \leqslant k \leqslant m, V_{k}^{m}$ reduces to $V_{k-1}^{m}$ in 4 Cremona moves. Proof. We have

$$
\begin{aligned}
& V_{k}^{m}=\left(P_{2 k+1} H_{2 m}+H_{2 k} P_{2 m} ; \frac{1}{2}\left(\left(2 H_{2 k}-P_{2 k}\right) H_{2 m}+H_{2 k} P_{2 m}\right)^{\times 4},\right. \\
& \left.\left(2 H_{2 k-1}-P_{2 k-1}\right) H_{2 m}+H_{2 k-1} P_{2 m},\left(P_{2 k} H_{2 m}\right)^{\times 2},(*)\right) \rightarrow \\
& \left(\left(-H_{2 k}+\frac{7}{2} P_{2 k}\right) H_{2 m}+\frac{1}{2} H_{2 k} P_{2 m} ;\left(H_{2 k-1} H_{2 m}\right)^{\times 3}\right. \text {, } \\
& \frac{1}{2}\left(\left(2 H_{2 k}-P_{2 k}\right) H_{2 m}+H_{2 k} P_{2 m}\right),\left(2 H_{2 k-1}-P_{2 k-1}\right) H_{2 m}+ \\
& \left.+H_{2 k-1} P_{2 m},\left(P_{2 k} H_{2 m}\right)^{\times 2},(*)\right) \rightsquigarrow \\
& \left(\left(-H_{2 k}+\frac{7}{2} P_{2 k}\right) H_{2 m}+\frac{1}{2} H_{2 k} P_{2 m} ; \frac{1}{2}\left(\left(2 H_{2 k}-P_{2 k}\right) H_{2 m}+H_{2 k} P_{2 m}\right)\right. \text {, } \\
& \left(2 H_{2 k-1}-P_{2 k-1}\right) H_{2 m}+H_{2 k-1} P_{2 m},\left(P_{2 k} H_{2 m}\right)^{\times 2} \text {, } \\
& \left.\left(H_{2 k-1} H_{2 m}\right)^{\times 3},(*)\right) \rightarrow \\
& \left(\frac{3}{2} P_{2 k} H_{2 m}+\frac{1}{2} H_{2 k-2} P_{2 m} ;\left(2 H_{2 k}-\frac{5}{2} P_{2 k}\right) H_{2 m}+\frac{1}{2} H_{2 k-2} P_{2 m}, P_{2 k-2} H_{2 m}\right. \text {, } \\
& \left.P_{2 k-1} H_{2 m}-H_{2 k-1} P_{2 m}, P_{2 k} H_{2 m},\left(H_{2 k-1} H_{2 m}\right)^{\times 3},(*)\right) \rightsquigarrow \\
& \left(\frac{3}{2} P_{2 k} H_{2 m}+\frac{1}{2} H_{2 k-2} P_{2 m} ; P_{2 k} H_{2 m},\left(H_{2 k-1} H_{2 m}\right)^{\times 3}\right. \text {, } \\
& \left.\left(2 H_{2 k}-\frac{5}{2} P_{2 k}\right) H_{2 m}+\frac{1}{2} H_{2 k-2} P_{2 m}, P_{2 k-2} H_{2 m}, P_{2 m-(2 k-1)},(*)\right) \rightarrow
\end{aligned}
$$




$$
\begin{aligned}
& \left(2 P_{2 k-1} H_{2 m}+H_{2 k-2} P_{2 m} ;\left(2 H_{2 k}-\frac{5}{2} P_{2 k}\right) H_{2 m}+\frac{1}{2} H_{2 k-2} P_{2 m},\right. \\
& \quad\left(-\frac{1}{2} P_{2 k-2} H_{2 m}+\frac{1}{2} H_{2 k-2} P_{2 m}\right)^{\times 2}, H_{2 k-1} H_{2 m}, \\
& \quad\left(2 H_{2 k}-\frac{5}{2} P_{2 k}\right) H_{2 m}+\frac{1}{2} H_{2 k-2} P_{2 m}, P_{2 k-2} H_{2 m}, \\
& \left.P_{2 m-(2 k-1)},(*)\right) \rightsquigarrow \\
& \left(2 P_{2 k-1} H_{2 m}+H_{2 k-2} P_{2 m} ; H_{2 k-1} H_{2 m},\right. \\
& \quad\left(\left(2 H_{2 k}-\frac{5}{2} P_{2 k}\right) H_{2 m}+\frac{1}{2} H_{2 k-2} P_{2 m}\right)^{\times 2}, P_{2 k-2} H_{2 m}, \\
& \left.\quad\left(\frac{1}{2} P_{2 m-(2 k-2)}\right)^{\times 2}, P_{2 m-(2 k-1)},(*)\right) \rightarrow \\
& \left(P_{2 k-1} H_{2 m}+H_{2 k-2} P_{2 m} ; P_{2 k-2} H_{2 m},\left(-\frac{1}{2} P_{2 k-2} H_{2 m}+\frac{1}{2} H_{2 k-2} P_{2 m}\right)^{\times 2},\right. \\
& \left.\quad P_{2 k-2} H_{2 m},\left(\frac{1}{2} P_{2 m-(2 k-2)}\right)^{\times 2}, P_{2 m-(2 k-1)},(*)\right) \\
& \left(P_{2 k-1} H_{2 m}+H_{2 k-2} P_{2 m} ;\left(P_{2 k-2} H_{2 m}\right)^{\times 2},\left(\frac{1}{2} P_{2 m-(2 k-2)}\right)^{\times 4},\right. \\
& \left.\quad P_{2 m-(2 k-1)},(*)\right) .
\end{aligned}
$$

Now, after reordering this last class, we obtain $V_{k-1}^{m}$ as required.

Lemma 5.9. $V_{2}^{m}$ reduces in 8 Cremona moves to the class

$$
V^{m}:=\left(H_{2 m} ; H_{2 m}-\frac{1}{2} P_{2 m},\left(\frac{1}{2} P_{2 m}\right)^{\times 3},\left(P_{2 m-1}\right)^{\times 2}, B_{1}^{m}\right) .
$$

Proof. We have

$$
\begin{aligned}
& V_{2}^{m}=\left(29 H_{2 m}+17 P_{2 m} ;\left(11 H_{2 m}+\frac{17}{2} P_{2 m}\right)^{\times 4}, 9 H_{2 m}+7 P_{2 m},\right. \\
& \left.\left(12 H_{2 m}\right)^{\times 2},\left(2 H_{2 m}+\frac{3}{2} P_{2 m}\right)^{\times 4}, H_{2 m}+P_{2 m}, H_{2 m}+\frac{1}{2} P_{2 m},(*)\right) ; \\
& \left(25 H_{2 m}+\frac{17}{2} P_{2 m} ; 11 H_{2 m}+\frac{17}{2} P_{2 m}, 9 H_{2 m}+7 P_{2 m},\left(12 H_{2 m}\right)^{\times 2},\right. \\
& \left.\quad\left(7 H_{2 m}\right)^{\times 3},\left(2 H_{2 m}+\frac{3}{2} P_{2 m}\right)^{\times 4}, H_{2 m}+P_{2 m}, H_{2 m}+\frac{1}{2} P_{2 m},(*)\right) ; \\
& \left(18 H_{2 m}+\frac{3}{2} P_{2 m} ; 12 H_{2 m},\left(7 H_{2 m}\right)^{\times 3}, 4 H_{2 m}+\frac{3}{2} P_{2 m},\left(2 H_{2 m}+\frac{3}{2} P_{2 m}\right)^{\times 4},\right. \\
& \left.2 H_{2 m}, H_{2 m}+P_{2 m}, H_{2 m}+\frac{1}{2} P_{2 m}, P_{2 m-3},(*)\right) ; \\
& \left(10 H_{2 m}+3 P_{2 m} ; 7 H_{2 m},\left(4 H_{2 m}+\frac{3}{2} P_{2 m}\right)^{\times 2},\left(2 H_{2 m}+\frac{3}{2} P_{2 m}\right)^{\times 4},\right. \\
& \left.2 H_{2 m}, H_{2 m}+P_{2 m}, H_{2 m}+\frac{1}{2} P_{2 m},\left(\frac{1}{2} P_{2 m-2}\right)^{\times 2}, P_{2 m-3},(*)\right) ; \\
& \left(5 H_{2 m}+3 P_{2 m} ;\left(2 H_{2 m}+\frac{3}{2} P_{2 m}\right)^{\times 4},\left(2 H_{2 m}\right)^{\times 2}, H_{2 m}+P_{2 m},\right. \\
& \left.H_{2 m}+\frac{1}{2} P_{2 m},\left(\frac{1}{2} P_{2 m-2}\right)^{\times 2}, P_{2 m-3},(*)\right) ;
\end{aligned}
$$




$$
\begin{aligned}
& \left(4 H_{2 m}+\frac{3}{2} P_{2 m} ; 2 H_{2 m}+\frac{3}{2} P_{2 m},\left(2 H_{2 m}\right)^{\times 2}, H_{2 m}+P_{2 m}, H_{2 m}+\frac{1}{2} P_{2 m},\right. \\
& \left.\quad\left(H_{2 m}\right)^{\times 3},\left(\frac{1}{2} P_{2 m-2}\right)^{\times 4}, P_{2 m-3},(*)\right) ; \\
& \left(2 H_{2 m}+\frac{3}{2} P_{2 m} ; H_{2 m}+P_{2 m}, H_{2 m}+\frac{1}{2} P_{2 m}, \frac{3}{2} P_{2 m},\left(H_{2 m}\right)^{\times 3},\right. \\
& \left.\quad\left(\frac{1}{2} P_{2 m-2}\right)^{\times 4}, P_{2 m-3},(*)\right) ; \\
& \left(2 H_{2 m} ;\left(H_{2 m}\right)^{\times 3}, H_{2 m}-\frac{1}{2} P_{2 m}, P_{2 m-1},\left(\frac{1}{2} P_{2 m-2}\right)^{\times 4}, P_{2 m-3},(*)\right) ; \\
& \left(H_{2 m} ; H_{2 m}-\frac{1}{2} P_{2 m}, P_{2 m-1},\left(\frac{1}{2} P_{2 m-2}\right)^{\times 4}, P_{2 m-3},(*)\right) .
\end{aligned}
$$

After reordering this last class, we obtain $V^{m}$ as required.

Lemma 5.10. For all $m \geqslant 3, V^{m}$ reduces in 5 Cremona moves to $V^{m-1}$.

Proof. We have

$$
\begin{aligned}
& V^{m}=\left(H_{2 m} ; H_{2 m}-\frac{1}{2} P_{2 m},\left(\frac{1}{2} P_{2 m}\right)^{\times 3},\left(P_{2 m-1}\right)^{\times 2},\left(\frac{1}{2} P_{2 m-2}\right)^{\times 8},(*)\right) ; \\
& \left(H_{2 m}-\frac{1}{2} P_{2 m} ; \frac{1}{2} P_{2 m},\left(P_{2 m-1}\right)^{\times 3},\left(\frac{1}{2} P_{2 m-2}\right)^{\times 8},(*)\right) ; \\
& \left(\frac{1}{2} P_{2 m} ; P_{2 m-1},\left(\frac{1}{2} P_{2 m-2}\right)^{\times 9},(*)\right) ; \\
& \left(P_{2 m-1} ; P_{2 m-1}-\frac{1}{2} P_{2 m-2},\left(\frac{1}{2} P_{2 m-2}\right)^{\times 7},(*)\right) ; \\
& \left(P_{2 m-1}-\frac{1}{2} P_{2 m-2} ; H_{2 m-2},\left(\frac{1}{2} P_{2 m-2}\right)^{\times 5},(*)\right) ; \\
& \left(H_{2 m-2} ; H_{2 m-2}-\frac{1}{2} P_{2 m-2},\left(\frac{1}{2} P_{2 m-2}\right)^{\times 3},(*)\right) .
\end{aligned}
$$

After reordering this class, we obtain $V^{m-1}$ as required.

Lemma 5.11. $V^{2}$ reduces in 8 Cremona moves to $(0 ;-1)$.

Proof. We have

$$
\begin{aligned}
& V^{2}=\left(17 ; 11,6^{\times 3}, 5^{\times 2}, 1^{\times 11}\right) ; \\
& \left(11 ; 6,5^{\times 3}, 1^{\times 11}\right) ; \\
& \left(6 ; 5,1^{\times 12}\right) ; \\
& \left(5 ; 4,1^{\times 10}\right) ; \\
& \left(4 ; 3,1^{\times 8}\right) ; \\
& \left(3 ; 2,1^{\times 6}\right) ; \\
& \left(2 ; 1^{\times 5}\right) ; \\
& \left(1 ; 1^{\times 2}\right) ; \\
& (0 ;-1) .
\end{aligned}
$$


5.2.2. The classes $E\left(\alpha_{2 m-1}\right)$ reduce to $(0 ;-1)$. One readily checks that the classes $E\left(\alpha_{2 m-1}\right)$ reduce to $(0 ;-1)$ for $m=1,2$. In the following, we reduce the classes $E\left(\alpha_{2 m-1}\right)$ for $m \geqslant 3$.

Lemma 5.12. The continued fraction expansion of $\alpha_{2 m-1}$ is

$$
\left[5 ;\{1,4\}^{\times(m-2)}, 1,3,1,1,\{4,1\}^{\times(m-1)}\right] .
$$

Moreover, if $v_{j}:=\left(2 H_{j}+P_{j}\right) H_{2 m}-H_{j} P_{2 m}$, then

$$
\begin{aligned}
E\left(\alpha_{2 m-1}\right)= & \left(P_{2 m-1} H_{2 m}, P_{2 m-1} H_{2 m}\right. \\
& \left(\frac{1}{2} v_{2 m-2}\right)^{\times 5}, v_{2 m-3},\left(\frac{1}{2} v_{2 m-4}\right)^{\times 4}, \ldots, v_{3},\left(\frac{1}{2} v_{2}\right)^{\times 4} \\
& v_{1},\left(H_{2 m}-\frac{1}{2} P_{2 m}\right)^{\times 3}, \frac{1}{2} P_{2 m}, P_{2 m-1}, \\
& \left.\left(\frac{1}{2} P_{2 m-2}\right)^{\times 4}, P_{2 m-3}, \ldots,\left(\frac{1}{2} P_{2}\right)^{\times 4}, P_{1}, 1\right)
\end{aligned}
$$

Proof. The first terms of $W^{\prime}\left(\alpha_{2 m-1}\right)$ are $\left(\frac{1}{2} v_{2 m-2}\right)^{\times 5}$ since $\frac{1}{2} v_{2 m-2}=2 P_{2 m-1}^{2}$ and $5<\alpha_{2 m-1}<6$ for $m \geqslant 2$. Before determining the next terms, we prove that for all $k \geqslant 1, v_{2 k+1}>\frac{1}{2} v_{2 k}>v_{2 k-1}$. Indeed

$$
\begin{aligned}
v_{2 k+1} & =\left(H_{2 k+1}+P_{2 k+1}\right) P_{2 m}+\left(2 H_{2 k+1}+P_{2 k+1}\right) P_{2 m-1} \\
& =\left(5 P_{2 k}+2 P_{2 k-1}\right) P_{2 m}+\left(8 P_{2 k}+3 P_{2 k-1}\right) P_{2 m-1} \\
& >\left(P_{2 k}+\frac{1}{2} P_{2 k-1}\right) P_{2 m}+\left(\frac{3}{2} P_{2 k}+P_{2 k-1}\right) P_{2 m-1} \\
& =\frac{1}{2}\left(H_{2 k}+P_{2 k}\right) P_{2 m}+\frac{1}{2}\left(2 H_{2 k}+P_{2 k}\right) P_{2 m-1} \\
& =\frac{1}{2} v_{2 k} \\
& =\left(\frac{5}{2} P_{2 k-1}+P_{2 k-2}\right) P_{2 m}+\left(3 P_{2 k-1}+\frac{7}{2} P_{2 k-2}+P_{2 k-3}\right) P_{2 m-1} \\
& >\left(2 P_{2 k-1}+P_{2 k-2}\right) P_{2 m}+\left(3 P_{2 k-1}+2 P_{2 k-2}\right) P_{2 m-1} \\
& =\left(H_{2 k-1}+P_{2 k-1}\right) P_{2 m}+\left(2 H_{2 k-1}+P_{2 k-1}\right) P_{2 m-1} \\
& =v_{2 k-1} .
\end{aligned}
$$


So the next term of $W^{\prime}\left(\alpha_{2 m-1}\right)$ is $H_{2 m}^{2}-5\left(2 P_{2 m-1}^{2}\right)=v_{2 m-3}<\frac{1}{2} v_{2 m-2}$. Moreover, for all $k \geqslant 1$,

$$
\begin{aligned}
v_{2 k+1}-4\left(\frac{1}{2} v_{2 k}\right) & =\left(2 H_{2 k+1}+P_{2 k+1}-4 H_{2 k}-2 P_{2 k}\right) H_{2 m} \\
& -\left(H_{2 k+1}-2 H_{2 k}\right) P_{2 m} \\
& =\left(2 H_{2 k-1}+P_{2 k-1}\right) H_{2 m}-H_{2 k-1} P_{2 m}=v_{2 k-1}<\frac{1}{2} v_{2 k} ; \\
\frac{1}{2} v_{2 k}-v_{2 k-1} & =\left(H_{2 k}+\frac{1}{2} P_{2 k}-2 H_{2 k-1}-P_{2 k-1}\right) H_{2 m} \\
& -\left(\frac{1}{2} H_{2 k}-H_{2 k-1}\right) P_{2 m} \\
& =\frac{1}{2}\left(2 H_{2 k-2}+P_{2 k-2}\right) H_{2 m}-\frac{1}{2} H_{2 k-2} P_{2 m}=\frac{1}{2} v_{2 k-2} \\
& <v_{2 k-1} .
\end{aligned}
$$

This proves that the first terms of $W^{\prime}\left(\alpha_{2 m-1}\right)$ are

$$
\left(\frac{1}{2} v_{2 m-2}\right)^{\times 5}, v_{2 m-3},\left(\frac{1}{2} v_{2 m-4}\right)^{\times 4}, \ldots, v_{3},\left(\frac{1}{2} v_{2}\right)^{\times 4}, v_{1} .
$$

The next three terms are $\left(H_{2 m}-\frac{1}{2} P_{2 m}\right)^{\times 3}, \frac{1}{2} P_{2 m}, P_{2 m-1}$ since

$$
\begin{aligned}
\frac{1}{2} v_{2}-v_{1} & =H_{2 m}-\frac{1}{2} P_{2 m}<3 H_{2 m}-P_{2 m}=v_{1} ; \\
v_{1}-3\left(H_{2 m}-\frac{1}{2} P_{2 m}\right) & =\frac{1}{2} P_{2 m}<H_{2 m}-\frac{1}{2} P_{2 m} ; \\
H_{2 m}-\frac{1}{2} P_{2 m}-\frac{1}{2} P_{2 m} & =P_{2 m-1}<\frac{1}{2} P_{2 m} .
\end{aligned}
$$

Since the last terms are the same as those of $W^{\prime}\left(\alpha_{2 m}\right)$, the lemma is proved.

Let us introduce again some notations.

Definition 5.13. Set

$$
\begin{aligned}
\hat{A}_{k}^{m}:= & \left(\left(\frac{1}{2} v_{2 k-2}\right)^{\times 4}, v_{2 k-3},\left(H_{2 k-1} H_{2 m}-H_{2 k-1} P_{2 m}\right)^{\times 2},\left(\frac{1}{2} v_{2 k-4}\right)^{\times 4},\right. \\
& \left.v_{2 k-5}, \ldots,\left(\frac{1}{2} v_{2}\right)^{\times 4}, v_{1},\left(H_{2 m}-\frac{1}{2} P_{2 m}\right)^{\times 3}, \frac{1}{2} P_{2 m}, P_{2 m-1}\right),
\end{aligned}
$$




$$
\begin{aligned}
\hat{B}_{k}^{m}:= & \left(\left(\frac{1}{2} P_{2 m-2}\right)^{\times 4}, P_{2 m-3}, \ldots,\left(\frac{1}{2} P_{2 m-2 k+2}\right)^{\times 4}, P_{2 m-2 k+1},\right. \\
& \left.\left(\frac{1}{2} P_{2 m-2 k}\right)^{\times 8},\left(P_{2 m-2 k-1}\right)^{\times 2}, \ldots,\left(\frac{1}{2} P_{2}\right)^{\times 8},\left(P_{1}\right)^{\times 2}, 1\right), \\
\hat{V}_{k}^{m}:= & \left(P_{2 k} H_{2 m}+H_{2 k-1} P_{2 m} ; \hat{A}_{k}^{m}, \hat{B}_{k}^{m}\right) .
\end{aligned}
$$

Note that $\hat{B}_{k}^{m}$ is actually equal to the vector $B_{k}^{m}$ that we used in the reduction of the classes $E\left(\alpha_{2 m}\right)$. Here, $\hat{A}_{k}^{m}$ has the structure

$$
\left[4,1,2,\{4,1\}^{\times(k-2)}, 3,1,1\right]
$$

and $\hat{B}_{k}^{m}$ has the structure

$$
\left[\{4,1\}^{\times(k-1)},\{8,2\}^{\times(m-k)}, 1\right] .
$$

We use again the convention that if $k=m, \hat{B}_{m}^{m}$ has the structure $\left[\{4,1\}^{\times(m-1)}\right.$, $1]$ and that if $k=1, \hat{B}_{1}^{m}$ has the structure $\left[\{8,2\}^{\times(m-1)}, 1\right]$.

Lemma 5.14. The image of $E\left(\alpha_{2 m-1}\right)$ by $\varphi_{*}$ is the class

$$
\varphi_{*}\left(E\left(\alpha_{2 m-1}\right)\right)=\left(P_{2 m} H_{2 m}-H_{2 m-1} P_{2 m} ; \hat{A}_{m}^{m}, \hat{B}_{m}^{m}\right)=\hat{V}_{m}^{m} .
$$

Proof. The first terms of $E\left(\alpha_{2 m-1}\right)$ are

$$
\left(P_{2 m-1} H_{2 m}, P_{2 m-1} H_{2 m} ; \frac{1}{2} v_{2 m-2},(*)\right) \text {. }
$$

Since $\frac{1}{2} v_{2 m-2}=2 P_{2 m-1}^{2}$, we get

$$
\begin{aligned}
\varphi_{*}\left(E\left(\alpha_{2 m-1}\right)\right)= & \left(2 P_{2 m-1} H_{2 m}-2 P_{2 m-1}^{2} ;\left(P_{2 m-1} H_{2 m}-2 P_{2 m-1}^{2}\right)^{\times 2},(*)\right) \\
= & \left(P_{2 m} H_{2 m}-H_{2 m-1} P_{2 m} ;\right. \\
& \left.\left(H_{2 m-1} H_{2 m}-H_{2 m-1} P_{2 m}\right)^{\times 2},(*)\right) .
\end{aligned}
$$

After reordering, this last class is $\left(P_{2 m} H_{2 m}-H_{2 m-1} P_{2 m} ; \hat{A}_{m}^{m}, \hat{B}_{m}^{m}\right)=\hat{V}_{m}^{m}$ as required.

Lemma 5.15. For all $3 \leqslant k \leqslant m, \hat{V}_{k}^{m}$ reduces to $\hat{V}_{k-1}^{m}$ in 4 Cremona moves. 
Proof. We have

$$
\begin{aligned}
& \hat{V}_{k}^{m}=\left(P_{2 k} H_{2 m}-H_{2 k-1} P_{2 m} ; \frac{1}{2}\left(\left(2 H_{2 k-2}+P_{2 k-2}\right) H_{2 m}-H_{2 k-2} P_{2 m}\right)^{\times 4},\right. \\
& \left(2 H_{2 k-3}+P_{2 k-3}\right) H_{2 m}-H_{2 k-3} P_{2 m} \text {, } \\
& \left.\left(H_{2 k-1} H_{2 m}-H_{2 k-1} P_{2 m}\right)^{\times 2},(*)\right) \rightarrow \\
& \left(\left(H_{2 k-2}+\frac{9}{2} P_{2 k-2}\right) H_{2 m}-\left(\frac{1}{2} H_{2 k-2}+4 P_{2 k-2}\right) P_{2 m}\right. \text {; } \\
& \left(2 P_{2 k-2} H_{2 m}-2 P_{2 k-2} P_{2 m}\right)^{\times 3} \text {, } \\
& \frac{1}{2}\left(\left(2 H_{2 k-2}+P_{2 k-2}\right) H_{2 m}-H_{2 k-2} P_{2 m}\right) \text {, } \\
& \left(2 H_{2 k-3}+P_{2 k-3}\right) H_{2 m}-H_{2 k-3} P_{2 m} \text {, } \\
& \left.\left(H_{2 k-1} H_{2 m}-H_{2 k-1} P_{2 m}\right)^{\times 2},(*)\right) \rightsquigarrow \\
& \left(\left(H_{2 k-2}+\frac{9}{2} P_{2 k-2}\right) H_{2 m}-\left(\frac{1}{2} H_{2 k-2}+4 P_{2 k-2}\right) P_{2 m}\right. \text {; } \\
& \frac{1}{2}\left(\left(2 H_{2 k-2}+P_{2 k-2}\right) H_{2 m}-H_{2 k-2} P_{2 m}\right) \text {, } \\
& \left(2 H_{2 k-3}+P_{2 k-3}\right) H_{2 m}-H_{2 k-3} P_{2 m},\left(H_{2 k-1} H_{2 m}-H_{2 k-1} P_{2 m}\right)^{\times 2} \text {, } \\
& \left.\left(2 P_{2 k-2} H_{2 m}-2 P_{2 k-2} P_{2 m}\right)^{\times 3},(*)\right) \rightarrow \\
& \left(\left(H_{2 k-2}+\frac{7}{2} P_{2 k-2}\right) H_{2 m}-\left(\frac{1}{2} H_{2 k-2}+4 P_{2 k-2}\right) P_{2 m}\right. \text {; } \\
& \left(H_{2 k-2}-\frac{1}{2} P_{2 k-2}\right) H_{2 m}-\frac{1}{2} H_{2 k-2} P_{2 m}, H_{2 k-3} H_{2 m}-H_{2 k-3} P_{2 m} \text {, } \\
& P_{2 k-1} H_{2 m}-H_{2 k-1} P_{2 m}, H_{2 k-1} H_{2 m}-H_{2 k-1} P_{2 m} \text {, } \\
& \left.\left(2 P_{2 k-2} H_{2 m}-2 P_{2 k-2} P_{2 m}\right)^{\times 3},(*)\right) \rightsquigarrow \\
& \left(\left(H_{2 k-2}+\frac{7}{2} P_{2 k-2}\right) H_{2 m}-\left(\frac{1}{2} H_{2 k-2}+4 P_{2 k-2}\right) P_{2 m}\right. \text {; } \\
& H_{2 k-1} H_{2 m}-H_{2 k-1} P_{2 m},\left(2 P_{2 k-2} H_{2 m}-2 P_{2 k-2} P_{2 m}\right)^{\times 3} \text {, } \\
& \left(H_{2 k-2}-\frac{1}{2} P_{2 k-2}\right) H_{2 m}-\frac{1}{2} H_{2 k-2} P_{2 m}, H_{2 k-3} H_{2 m}-H_{2 k-3} P_{2 m} \text {, } \\
& \left.P_{2 m-(2 k-1)},(*)\right) \rightarrow \\
& \left(P_{2 k-1} H_{2 m}-2 P_{2 k-2} P_{2 m} ;\left(H_{2 k-2}-\frac{1}{2} P_{2 k-2}\right) H_{2 m}-\frac{1}{2} H_{2 k-2} P_{2 m}\right. \text {, } \\
& \left(-\frac{1}{2} P_{2 k-2} H_{2 m}+\frac{1}{2} H_{2 k-2} P_{2 m}\right)^{\times 2}, 2 P_{2 k-2} H_{2 m}-2 P_{2 k-2} P_{2 m}, \\
& \left(H_{2 k-2}-\frac{1}{2} P_{2 k-2}\right) H_{2 m}-\frac{1}{2} H_{2 k-2} P_{2 m}, H_{2 k-3} H_{2 m}-H_{2 k-3} P_{2 m} \text {, } \\
& \left.P_{2 m-(2 k-1)},(*)\right) \rightsquigarrow \\
& \left(P_{2 k-1} H_{2 m}-2 P_{2 k-2} P_{2 m} ; 2 P_{2 k-2} H_{2 m}-2 P_{2 k-2} P_{2 m}\right. \text {, } \\
& \left(\left(H_{2 k-2}-\frac{1}{2} P_{2 k-2}\right) H_{2 m}-\frac{1}{2} H_{2 k-2} P_{2 m}\right)^{\times 2}, H_{2 k-3} H_{2 m}-H_{2 k-3} P_{2 m} \text {, } \\
& \left.\left(\frac{1}{2} P_{2 m-(2 k-2)}\right)^{\times 2}, P_{2 m-(2 k-1)},(*)\right) \rightarrow \\
& \left(P_{2 k-2} H_{2 m}-H_{2 k-3} P_{2 m} ; H_{2 k-3} H_{2 m}-H_{2 k-3} P_{2 m}\right. \text {, } \\
& \left(-\frac{1}{2} P_{2 k-2} H_{2 m}+\frac{1}{2} H_{2 k-2} P_{2 m}\right)^{\times 2}, H_{2 k-3} H_{2 m}-H_{2 k-3} P_{2 m}, \\
& \left.\left(\frac{1}{2} P_{2 m-(2 k-2)}\right)^{\times 2}, P_{2 m-(2 k-1)},(*)\right) \rightsquigarrow
\end{aligned}
$$




$$
\begin{aligned}
& \left(P_{2 k-2} H_{2 m}-H_{2 k-3} P_{2 m} ;\left(H_{2 k-3} H_{2 m}-H_{2 k-3} P_{2 m}\right)^{\times 2},\right. \\
& \left.\quad\left(\frac{1}{2} P_{2 m-(2 k-2)}\right)^{\times 4}, P_{2 m-(2 k-1)},(*)\right) .
\end{aligned}
$$

Now, after reordering this last class, we obtain $\hat{V}_{k-1}^{m}$ as required.

Lemma 5.16. $\hat{V}_{2}^{m}$ reduces in 5 Cremona moves to the class

$$
\hat{V}^{m}:=\left(H_{2 m}-\frac{1}{2} P_{2 m} ; \frac{1}{2} P_{2 m},\left(P_{2 m-1}\right)^{\times 3}, \hat{B}_{1}^{m}\right) .
$$

Proof. We have

$$
\begin{aligned}
& \hat{V}_{2}^{m}=\left(12 H_{2 m}-7 P_{2 m} ;\left(4 H_{2 m}-\frac{3}{2} P_{2 m}\right)^{\times 4}, 3 H_{2 m}-P_{2 m},\right. \\
& \left.\quad\left(7 H_{2 m}-7 P_{2 m}\right)^{\times 2},\left(H_{2 m}-\frac{1}{2} P_{2 m}\right)^{\times 3},(*)\right) ; \\
& \left(12 H_{2 m}-\frac{19}{2} P_{2 m} ; 4 H_{2 m}-\frac{3}{2} P_{2 m}, 3 H_{2 m}-P_{2 m},\left(7 H_{2 m}-7 P_{2 m}\right)^{\times 2},\right. \\
& \left.\quad\left(4 H_{2 m}-4 P_{2 m}\right)^{\times 3},\left(H_{2 m}-\frac{1}{2} P_{2 m}\right)^{\times 3},(*)\right) ; \\
& \left(10 H_{2 m}-\frac{19}{2} P_{2 m} ; 7 H_{2 m}-7 P_{2 m},\left(4 H_{2 m}-4 P_{2 m}\right)^{\times 3}, 2 H_{2 m}-\frac{3}{2} P_{2 m},\right. \\
& \left.\quad\left(H_{2 m}-\frac{1}{2} P_{2 m}\right)^{\times 3}, P_{2 m-1}, P_{2 m-3},(*)\right) ; \\
& \left(5 H_{2 m}-4 P_{2 m} ; 4 H_{2 m}-4 P_{2 m},\left(2 H_{2 m}-\frac{3}{2} P_{2 m}\right)^{\times 2},\left(H_{2 m}-\frac{1}{2} P_{2 m}\right)^{\times 3},\right. \\
& \left.\quad P_{2 m-1},\left(\frac{1}{2} P_{2 m-2}\right)^{\times 2}, P_{2 m-3},(*)\right) ; \\
& \left(2 H_{2 m}-P_{2 m} ;\left(H_{2 m}-\frac{1}{2} P_{2 m}\right)^{\times 3},\left(P_{2 m-1}\right)^{\times 2},\left(\frac{1}{2} P_{2 m-2}\right)^{\times 4}, P_{2 m-3},(*)\right) ; \\
& \left(H_{2 m}-\frac{1}{2} P_{2 m} ;\left(H_{2 m}-\frac{1}{2} P_{2 m}\right)^{\times 3},\left(P_{2 m-1}\right)^{\times 2},\left(\frac{1}{2} P_{2 m-2}\right)^{\times 4}, P_{2 m-3},(*)\right) .
\end{aligned}
$$

After reordering this last class, we obtain $\hat{V}^{m}$ as required.

Lemma 5.17. For all $m \geqslant 3, \hat{V}^{m}$ reduces in 5 Cremona moves to $\hat{V}^{m-1}$.

Proof. We have

$$
\begin{aligned}
& \hat{V}^{m}=\left(H_{2 m}-\frac{1}{2} P_{2 m} ; \frac{1}{2} P_{2 m},\left(P_{2 m-1}\right)^{\times 3},\left(\frac{1}{2} P_{2 m-2}\right)^{\times 8},(*)\right) ; \\
& \left(\frac{1}{2} P_{2 m} ; P_{2 m-1},\left(\frac{1}{2} P_{2 m-2}\right)^{\times 9},(*)\right) ; \\
& \left(P_{2 m-1} ; P_{2 m-1}-\frac{1}{2} P_{2 m-2},\left(\frac{1}{2} P_{2 m-2}\right)^{\times 7},(*)\right) ; \\
& \left(P_{2 m-1}-\frac{1}{2} P_{2 m-2} ; H_{2 m-2},\left(\frac{1}{2} P_{2 m-2}\right)^{\times 5},(*)\right) ; \\
& \left(H_{2 m-2} ; H_{2 m-2}-\frac{1}{2} P_{2 m-2},\left(\frac{1}{2} P_{2 m-2}\right)^{\times 3},(*)\right) ;
\end{aligned}
$$


$\left(H_{2 m-2}-\frac{1}{2} P_{2 m-2} ; \frac{1}{2} P_{2 m-2}, P_{2 m-3},(*)\right)$.

After reordering this class, we obtain $\hat{V}^{m-1}$ as required.

Lemma 5.18. $\hat{V}^{2}$ reduces in 7 Cremona moves to $(0 ;-1)$.

Proof. We have

$$
\begin{aligned}
& \hat{V}^{2}=\left(11 ; 6,5^{\times 3}, 1^{\times 11}\right) ; \\
& \left(6 ; 5,1^{\times 12}\right) ; \\
& \left(5 ; 4,1^{\times 10}\right) ; \\
& \left(4 ; 3,1^{\times 8}\right) ; \\
& \left(3 ; 2,1^{\times 6}\right) \\
& \left(2 ; 1^{\times 5}\right) ; \\
& \left(1 ; 1^{\times 2}\right) ; \\
& (0 ;-1) .
\end{aligned}
$$

\section{The interval $\left[\sigma^{2}, 6\right]$}

In this section we prove that $c(a)=\frac{a+1}{4}$ on the interval $\left[\sigma^{2}, 6\right]$, which is a part of Theorem 7.2 Notice that the class $\left(2,2 ; 2,1^{\times 5}\right)$ gives the constraint $\frac{a+1}{4}$ on $\left[\sigma^{2}, 6\right]$. If thus suffices to show that no class gives a stronger constraint. We begin by showing this on the interval $\left[5 \frac{12}{13}, 6\right]$, and will then spend some efforts to extend it to $\left[\sigma^{2}, 6\right]$.

Proposition 6.1. For $a \in\left[5 \frac{12}{13}, 6\right]$, we have $c(a)=\frac{a+1}{4}$.

Proof. Write $a=5+x \in\left[5 \frac{12}{13}, 6\left[\right.\right.$. Then $w(a)=\left(1^{\times 5}, x, w_{7}, \ldots, w_{M}\right)$. Since $x \geqslant \frac{12}{13}, 1-x \leqslant \frac{x}{9}$, thus at least the first nine of the weights $w_{7}, \ldots, w_{M}$ are equal. Then, by Corollary 1.2.4 in [MS], the $M-6$ balls of weights $w_{7}, \ldots, w_{M}$ fully fill the ball of weight $\lambda$, where $a=5+x^{2}+\lambda^{2}$. Thus to prove that $c(a)=$ $\frac{a+1}{4}$ on $\left[\sigma^{2}, 6\right]$, it suffices to check that the seven balls of weights $1^{\times 5}, x, \lambda$ embed into the cube $C\left(\frac{(5+x)+1}{4}\right)$, or in other words, that the finite number of classes of $\mathcal{E}_{M}$ with $M \leqslant 7$ don't give any embedding constraint stronger than $\frac{a+1}{4}$ for these seven balls. 
This is clear for the classes belonging to $\mathcal{E}_{M}$ with $M \leqslant 6$. The strongest constraint of $\mathcal{E}_{7}$ comes from the class $\left(4,3 ; 2^{\times 6}, 1\right)$ for which

$$
\mu\left(4,3 ; 2^{\times 6}, 1\right)(5+x)=\frac{10+2 x+\lambda}{7}
$$

Notice that $\frac{10+2 x+\lambda}{7} \leqslant \frac{(5+x)+1}{4}$ if and only if $\lambda \leqslant \frac{2-x}{4}$. Recall that $a=5+x=$ $5+x^{2}+\lambda^{2}$, whence $\lambda^{2}=x-x^{2}$. Since $x-x^{2} \leqslant\left(\frac{2-x}{4}\right)^{2}$ for $x \in\left[\frac{12}{13}, 1\right]$. Thus the class $\left(4,3 ; 2^{\times 6}, 1\right)$ indeed gives no stronger constraint than $\frac{a+1}{4}$. Finally, by continuity of $c, c(6)=\frac{6+1}{4}=\frac{7}{4}$.

Proposition 6.2. Let $(d, e ; m) \in \mathcal{E}$ be a class such that $\mu(d, e ; m)(a)>\frac{a+1}{4} \geqslant$ $\sqrt{\frac{a}{2}}$ for some $a \in\left[\sigma^{2}, 6\right]$. Then

(i) $d<\frac{2 \sqrt{a}}{\sqrt{a^{2}-6 a+1}}$ for a class of the form $(d, d ; m)$ and $d<\frac{\sqrt{2 a}}{\sqrt{a^{2}-6 a+1}}$ for $a$ class of the form $\left(d+\frac{1}{2}, d-\frac{1}{2} ; m\right)$.

(ii) Moreover, if we denote $\lambda^{2}:=1-\sum_{i=1}^{M} \varepsilon_{i}^{2} \quad$ (respectively $\lambda^{2}:=\frac{1}{2}-$ $\left.\sum_{i=1}^{M} \varepsilon_{i}^{2}\right)$ for a class of the form $(d, d ; m)$ (respectively for a class of the form $\left.\left(d+\frac{1}{2}, d-\frac{1}{2} ; m\right)\right)$, then $\lambda^{2}>d^{2} \sqrt{\frac{2}{a}} y(a)$.

Proof. (i) Let us first prove this for a class of the form $(d, d ; m)$. By Lemma 4.8 (i), we have

$$
\frac{a+1}{4}<\mu(d, d ; m)(a) \leqslant \sqrt{1+\frac{1}{2 d^{2}}} \sqrt{\frac{a}{2}} .
$$

Thus

$$
\frac{(a+1)^{2}}{8 a}-1<\frac{1}{2 d^{2}}
$$

and so

$$
d<\frac{2 \sqrt{a}}{\sqrt{a^{2}-6 a+1}} .
$$

Similarly, for a class of the form $\left(d+\frac{1}{2}, d-\frac{1}{2} ; m\right)$, we have

$$
\frac{a+1}{4}<\mu\left(d+\frac{1}{2}, d-\frac{1}{2} ; m\right)(a) \leqslant \sqrt{1+\frac{1}{4 d^{2}}} \sqrt{\frac{a}{2}}
$$

and thus

$$
d<\frac{\sqrt{2 a}}{\sqrt{a^{2}-6 a+1}} .
$$


(ii) For a class of the form $(d, d ; m)$, we have by Proposition 3.8 (i),

$$
\begin{aligned}
2 d^{2}+1 & =\langle m, m\rangle=\left\langle\frac{\sqrt{2} d}{\sqrt{a}} w(a)+\varepsilon, \frac{\sqrt{2} d}{\sqrt{a}} w(a)+\varepsilon\right\rangle \\
& =2 d^{2}+\frac{2 \sqrt{2} d}{\sqrt{a}}\langle w(a), \varepsilon\rangle+\langle\varepsilon, \varepsilon\rangle .
\end{aligned}
$$

Thus

$$
\langle w(a), \varepsilon\rangle=\underbrace{(1-\langle\varepsilon, \varepsilon\rangle)}_{=\lambda^{2}} \frac{1}{2 d} \sqrt{\frac{a}{2}} .
$$

On the other hand

$$
\begin{aligned}
\frac{a+1}{4} & <\mu(d, d ; m)(a)=\frac{\langle m, w(a)\rangle}{2 d}=\frac{1}{2 d}\left\langle\frac{\sqrt{2} d}{\sqrt{a}} w(a)+\varepsilon, w(a)\right\rangle \\
& =\sqrt{\frac{a}{2}}+\frac{1}{2 d}\langle\varepsilon, w(a)\rangle .
\end{aligned}
$$

Thus

$$
\langle\varepsilon, w(a)\rangle>2 d\left(\frac{a+1}{4}-\sqrt{\frac{a}{2}}\right)=\frac{1}{2} d \underbrace{(a+1-2 \sqrt{2 a})}_{=y(a)} .
$$

Inserting (6.1) in this inequality, we get

$$
\frac{\lambda^{2}}{2 d} \sqrt{\frac{a}{2}}>\frac{d}{2} y(a)
$$

and finally

$$
\lambda^{2}>d^{2} \sqrt{\frac{2}{a}} y(a) .
$$

Similarly, for a class $\left(d+\frac{1}{2}, d-\frac{1}{2} ; m\right)$, we have

$$
2 d^{2}+\frac{1}{2}=2 d^{2}+\frac{2 \sqrt{2} d}{\sqrt{a}}\langle w(a), \varepsilon\rangle+\langle\varepsilon, \varepsilon\rangle,
$$

So

$$
\langle w(a), \varepsilon\rangle=\underbrace{\left(\frac{1}{2}-\langle\varepsilon, \varepsilon\rangle\right)}_{=\lambda^{2}} \frac{1}{2 d} \sqrt{\frac{a}{2}} .
$$

The rest of the proof is then identical to the case of a class $(d, d ; m)$. 
Notice that the continued fraction of $\sigma^{2}=3+2 \sqrt{2}$ is $[5 ; 1,4,1,4, \ldots]$. We will now define the so called convergents $c_{k}$ of $\sigma^{2}$ and some other numbers $u_{k}(j)$ and $v_{k}(j)$ which will play a crucial role in the proof of Theorem 6.21

Definition 6.3. For all $k, j \geqslant 1$, set

$$
\begin{aligned}
c_{2 k-1} & :=\left[5 ;\{1,4\}^{\times(k-1)}, 1\right]=\left[5 ;\{1,4\}^{\times(k-2)}, 1,5\right], \\
c_{2 k} & :=\left[5 ;\{1,4\}^{\times k}\right], \\
u_{k}(j) & :=\left[5 ;\{1,4\}^{\times(k-1)}, 1,5, j\right], \\
v_{k}(j) & :=\left[5 ;\{1,4\}^{\times(k-1)}, 1, j\right] .
\end{aligned}
$$

Lemma 6.4. For all $k, j \geqslant 1$, we have the following relations written in lowest terms

(i) $c_{2 k-1}=\frac{\frac{1}{2} P_{2 k+2}}{\frac{1}{2} P_{2 k}}, \quad c_{2 k}=\frac{P_{2 k+3}}{P_{2 k+1}}$,

(ii) $u_{k}(j)=\frac{\frac{1}{2}\left(j P_{2 k+4}+P_{2 k+2}\right)}{\frac{1}{2}\left(j P_{2 k+2}+P_{2 k}\right)}$,

(iii) $v_{k}(j)=\frac{\frac{1}{2} j P_{2 k+2}+P_{2 k+1}}{\frac{1}{2} j P_{2 k}+P_{2 k-1}}$.

Proof. We use the fact that if $\left[a_{0} ; a_{1}, \ldots, a_{M}\right]$ is a continued fraction and $\frac{p_{k}}{q_{k}}:=\left[a_{0} ; a_{1}, \ldots, a_{k}\right]$ is its $k$-th convergent written in lowest terms, then for any real number $x$,

$$
\left[a_{0} ; a_{1}, \ldots, a_{k}, x\right]=\frac{x p_{k}+p_{k-1}}{x q_{k}+q_{k-1}}
$$

written in lowest terms.

(i) We argue by induction on $k$. Assertion (i) is clear for $k=1$. Assume it holds for $k-1$. Then

$$
c_{2 k-1}=\left[5 ;\{1,4\}^{\times(k-1)}, 1\right]=\frac{\frac{1}{2} P_{2 k}+P_{2 k+1}}{\frac{1}{2} P_{2 k-2}+P_{2 k-1}}=\frac{\frac{1}{2} P_{2 k+2}}{\frac{1}{2} P_{2 k}},
$$

and

$$
c_{2 k}=\left[5 ;\{1,4\}^{\times k}\right]=\frac{P_{2 k+1}+2 P_{2 k+2}}{P_{2 k-1}+2 P_{2 k}}=\frac{P_{2 k+3}}{P_{2 k+1}} .
$$

The proofs of (ii) and (iii) are then straightforward. 
Corollary 6.5. We have

(i) $c_{2}<c_{4}<\cdots<c_{2 k}<\cdots<\sigma^{2}<\cdots<c_{2 k+1}<\cdots<c_{3}<c_{1}$,

(ii) $c_{2 k+1}<\cdots<u_{k}(2)<u_{k}(1)=v_{k}(6)<v_{k}(7)<\cdots<c_{2 k-1}$.

Proof. (i) It is a property of convergents that the even convergents (respectively odd convergents) of a real number $a$ form an increasing sequence (respectively decreasing sequence) converging to $a$.

(ii) This follows from Lemma 6.4 and the identity $P_{2 k+1} P_{2 k-1}-P_{2 k}^{2}=1$ for all $k \geqslant 1$.

Lemma 6.6. To prove that a quadratic identity of the form

$$
Q(s):=\sum_{i, j \geqslant 0} a_{i j} P_{s+i} P_{s+j}+\sum_{j \geqslant 0} b_{j} P_{2 s+j}+(-1)^{s} c=0
$$

holds for all $s \geqslant 0$, it suffices to check it for three distinct values of $s$. Moreover, if $Q$ is homogeneous and linear (that is, $c=0$ and $a_{i j}=0$ for all $i, j$ ), it suffices to check it for two distinct values of $s$.

Proof. The proof is similar to the proof of Proposition 3.2.3 in [MS. The only part of the proof which is slightly different is the proof of their Lemma 3.2.2, which we have adapted in the following Lemma 6.7 .

Lemma 6.7. For all $i \geqslant 0$, there exists a finite number of rational coefficients $a_{i j}, c_{i}$ such that the identity

$$
P_{s+i} P_{s}=\sum_{j \geqslant 0} a_{i j} P_{2 s+j}+(-1)^{s} c_{i}
$$

holds for all $s \geqslant 0$. Moreover, $c_{i}=-\sum_{j \geqslant 0} a_{i j} P_{j}$.

Proof. In view of the relation

$$
P_{k}=2 P_{k-1}+P_{k-2}
$$

it suffices to prove it for two distinct values of $i$. We claim that for $i=0$ and $i=2$ we have the following relations for all $s \geqslant 0$ :

$$
\begin{gathered}
4 P_{s}^{2}=P_{2 s+1}-P_{2 s}-(-1)^{s}, \\
8 P_{s+2} P_{s}=P_{2 s+1}+P_{2 s+3}-6(-1)^{s},
\end{gathered}
$$


To prove this, we use the two well-known identities for Pell numbers,

$$
\begin{aligned}
P_{k}^{2} & =P_{k+1} P_{k-1}-(-1)^{k}, \\
P_{2 k-1} & =P_{k}^{2}+P_{k-1}^{2} .
\end{aligned}
$$

We start with $i=0$. The relation is true for $s=0$. Now, if $s \geqslant 1$, applying 6.2 to 6.3 gives

$$
8 P_{s}^{2}=P_{s+1}^{2}+2 P_{s}^{2}+P_{s-1}^{2}-2(-1)^{s}
$$

Using again 6.2, we get

$$
6 P_{s}^{2}=P_{s+1}^{2}+P_{s-1}^{2}-2(-1)^{s}=4 P_{s}^{2}+4 P_{s} P_{s-1}+2 P_{s-1}^{2}-2(-1)^{s} .
$$

Finally, applying once more 6.2 , we obtain

$$
2 P_{s}^{2}=2 P_{s-1}\left(2 P_{s}+P_{s-1}\right)-2(-1)^{s}=2 P_{s+1} P_{s-1}-2(-1)^{s},
$$

which is true by 6.5 .

For $i=2$, applying 6.5 to the LHS of (6.4), and 6.6 to the RHS, we obtain

$$
8 P_{s+1}^{2}+8(-1)^{s+1}=P_{s+2}^{2}+2 P_{s+1}^{2}+P_{s}^{2}-6(-1)^{s},
$$

which is equivalent to

$$
6 P_{s+1}^{2}=P_{s+2}^{2}+P_{s}^{2}-2(-1)^{s+1}
$$

which is true by 6.7 .

Finally, we easily check that the formula for $c_{0}$ and $c_{2}$ holds.

Corollary 6.8. For all $k, j \geqslant 1$, we have:

(i) If we abbreviate $u:=u_{k}(j)=: \frac{p}{q}$, then $q^{2}\left(u^{2}-6 u+1\right)=j^{2}+6 j+1$,

(ii) If $v:=v_{k}(j)=\frac{p}{q}$, then $q^{2}\left(v^{2}-6 v+1\right)=j^{2}-4 j-4$,

(iii) If we denote $\frac{p}{q}:=u_{k}(2)$, then $p^{2}-6 p q+q^{2}-16=1$, and if $\frac{p}{q}:=u_{k}(3)$, then $p^{2}-6 p q+q^{2}-12=16$,

(iv) If we denote $\frac{p}{q}:=v_{k}(6)$, then $5 p^{2}-30 p q+5 q^{2}-32=8$, and if $\frac{p}{q}:=v_{k}(7)$, then $3 p^{2}-18 p q+3 q^{2}-28=23$.

(v) $P_{2 m-k}=(-1)^{k+1}\left(P_{k} H_{2 m}-H_{k} P_{2 m}\right)$ for all $m \geqslant 0$ and $k \leqslant 2 m$. 
Proof. By Lemma 6.6 we only have to check the first four identities for three values of $k$. It is easy to see that they are true for $k=1,2,3$. Similarly, it suffices to check the last identity for three even and three odd values.

Lemma 6.9. Set $\varphi(a):=\frac{a+1}{4}$ and $\psi(a)=\sqrt{\frac{a}{2}}$. Then

(i) $\varphi\left(u_{k}(j+1)\right)>\psi\left(u_{k}(j)\right)$ for all $k, j \geqslant 1$,

(ii) $\varphi\left(v_{k}(j)\right)>\psi\left(v_{k}(j+1)\right)$ for all $k \geqslant 1, j \geqslant 6$.

Proof. (i) Abbreviate $u:=u_{k}(j+1)$ and $u^{\prime}:=u_{k}(j)$. Due to Corollary 6.8(i) we have

$$
u^{2}=\frac{(j+1)^{2}+6(j+1)+1}{q^{2}}+6 u-1 .
$$

We have to prove that $\frac{u+1}{4}>\sqrt{\frac{u^{\prime}}{2}}$ which is equivalent to

$$
u^{2}+2 u+1>8 u^{\prime}
$$

which becomes

$$
\frac{(j+1)^{2}+6(j+1)+1}{q^{2}}+8 u>8 u^{\prime},
$$

and finally

$$
8\left(u^{\prime}-u\right) q^{2}<(j+1)^{2}+6(j+1)+1
$$

By Lemma 6.4(ii)

$$
\begin{aligned}
u^{\prime}-u & =\frac{\frac{1}{2}\left(j P_{2 k+4}+P_{2 k+2}\right)}{\frac{1}{2}\left(j P_{2 k+2}+P_{2 k}\right)}-\frac{\frac{1}{2}\left((j+1) P_{2 k+4}+P_{2 k+2}\right)}{\frac{1}{2}\left((j+1) P_{2 k+2}+P_{2 k}\right)} \\
& =\frac{(j+1) P_{2 k+2}^{2}-(j+1) P_{2 k} P_{2 k+4}+j P_{2 k+4} P_{2 k}-j P_{2 k+2}^{2}}{\left((j+1) P_{2 k+2}+P_{2 k}\right)\left(j P_{2 k+2}+P_{2 k}\right)} \\
& =\frac{P_{2 k+2}^{2}-P_{2 k} P_{2 k+4}}{\left((j+1) P_{2 k+2}+P_{2 k}\right)\left(j P_{2 k+2}+P_{2 k}\right)} \\
& =\frac{4}{\left((j+1) P_{2 k+2}+P_{2 k}\right)\left(j P_{2 k+2}+P_{2 k}\right)}
\end{aligned}
$$

since $P_{2 k+2}^{2}-P_{2 k} P_{2 k+4}=4$ for all $k \geqslant 1$ by Lemma 6.6 . Inserting this in 6.8 gives

$$
\frac{8\left((j+1) P_{2 k+2}+P_{2 k}\right)}{j P_{2 k+2}+P_{2 k}}<(j+1)^{2}+6(j+1)+1
$$


since $q^{2}=\frac{1}{4}\left((j+1) P_{2 k+2}+P_{2 k}\right)^{2}$. This inequality is now true for all $k, j \geqslant 1$ since the left hand side is smaller than 16 , and the right hand side is bigger than 17 .

(ii) Abbreviate $v:=v_{k}(j)$ and $v^{\prime}:=v_{k}(j+1)$. Due to Corollary 6.8(ii), we have

$$
v^{2}=\frac{j^{2}-4 j-4}{q^{2}}+6 v-1 .
$$

We have to prove that $\frac{v+1}{4}>\sqrt{\frac{v^{\prime}}{2}}$ which is equivalent to

$$
v^{2}+2 v+1>8 v^{\prime}
$$

which becomes

$$
\frac{j^{2}-4 j-4}{q^{2}}+8 v>8 v^{\prime}
$$

and finally

$$
8\left(v^{\prime}-v\right) q^{2}<j^{2}-4 j-4
$$

By Lemma 6.4(iii)

$$
\begin{aligned}
v^{\prime}-v & =\frac{\frac{1}{2} j P_{2 k+2}+P_{2 k+1}}{\frac{1}{2} j P_{2 k}+P_{2 k-1}}-\frac{\frac{1}{2}(j+1) P_{2 k+2}+P_{2 k+1}}{\frac{1}{2}(j+1) P_{2 k}+P_{2 k-1}} \\
& =\frac{j P_{2 k-1} P_{2 k+2}+(j+1) P_{2 k} P_{2 k+1}-j P_{2 k} P_{2 k+1}-(j+1) P_{2 k-1} P_{2 k+2}}{2\left(\frac{1}{2} j P_{2 k}+P_{2 k-1}\right)\left(\frac{1}{2}(j+1) P_{2 k}+P_{2 k-1}\right)} \\
& =\frac{P_{2 k} P_{2 k+1}-P_{2 k-1} P_{2 k+2}}{2\left(\frac{1}{2} j P_{2 k}+P_{2 k-1}\right)\left(\frac{1}{2}(j+1) P_{2 k}+P_{2 k-1}\right)} \\
& =\frac{-1}{\left(\frac{1}{2} j P_{2 k}+P_{2 k-1}\right)\left(\frac{1}{2}(j+1) P_{2 k}+P_{2 k-1}\right)}
\end{aligned}
$$

since $P_{2 k} P_{2 k+1}-P_{2 k-1} P_{2 k+2}=-2$ for all $k \geqslant 1$ by Lemma 6.6. Inserting this into 6.9 gives

$$
-\frac{8\left(\frac{1}{2} j P_{2 k}+P_{2 k-1}\right)}{\frac{1}{2}(j+1) P_{2 k}+P_{2 k-1}}<j^{2}-4 j-4,
$$

since $q^{2}=\left(\frac{1}{2} j P_{2 k}+P_{2 k-1}\right)^{2}$. This inequality is now true for all $k \geqslant 1, j \geqslant 6$ since the left hand side is negative, and the right hand side is positive.

Definition 6.10. A point $a \in\left[\sigma^{2}, 6\right]$ is said to be regular if for all $(d, e ; m) \in$ $\mathcal{E}$ such that $l(m)=l(a)$, it holds that $\mu(d, e ; m)(a) \leqslant \frac{a+1}{4}$. 
Proposition 6.11. Assume that the points

$c_{2 k-1}$ for all $k \geqslant 1$,

$u_{k}(j)$ for all $k \geqslant 1, j \geqslant 2$,

$v_{k}(j)$ for all $k \geqslant 1, j \geqslant 6$

are regular. Then $c(a)=\frac{a+1}{4}$ on $\left[\sigma^{2}, 6\right]$.

Proof. Assume by contradiction that $c\left(a_{0}\right)>\frac{a_{0}+1}{4}$ for some $a_{0} \in\left[\sigma^{2}, 6\right]$. Since for all $\left.a \in] \sigma^{2}, 6\right]$, we have $c(a) \geqslant \frac{a+1}{4}>\sqrt{\frac{a}{2}}$, the function $c(a)$ is piecewise linear on $\left.] \sigma^{2}, 6\right]$ by Corollary 4.9 . Let $\left.S \subset\right] \sigma^{2}, 6[$ be the set of non-smooth points of $c$ on $] \sigma^{2}, 6\left[\right.$. Decompose this set as $S=S_{+} \cup S_{-}$, where $S_{+}$(respectively $S_{-}$) consists of the points $s \in S$ near which $c$ is concave (respectively convex). Since $c(s)>\frac{s+1}{4}$ for all for all $s \in S_{+}$, the biggest point of $S$ is in $S_{-}$because $c(a)=\frac{a+1}{4}$ for $a \in\left[5 \frac{12}{13}, 6\right]$ by Proposition 6.1 . And since $c\left(\sigma^{2}\right)=\frac{\sigma^{2}+1}{4}$, it follows that the set $S_{+}$is non-empty. Let $s_{0}=\max S_{+}$. Then $\left.s_{0} \in\right] \sigma^{2}, 6[$. By Corollary 4.9 (i) there exists $(d, e ; m) \in \mathcal{E}$ and $\varepsilon>0$ such that

$$
c(z)=\mu(d, e ; m)(z)
$$

on $\left[s_{0}, s_{0}+\varepsilon\left[\right.\right.$. Abbreviate $\mu(z):=\mu(d, e ; m)(z)$. Then, $\mu\left(s_{0}\right)=c\left(s_{0}\right)>\frac{s_{0}+1}{4}>$ $\sqrt{\frac{s_{0}}{2}}$. Let $I$ be the maximal open interval containing $s_{0}$ on which $\mu(z)>$ $\sqrt{\frac{z}{2}}$ for all $z \in I$. By Lemma 4.13 there exists a unique $s^{\prime} \in I$ with $l(m)=$ $l\left(s^{\prime}\right)$, and $l(m)<l(z)$ for all other $z \in I$. Moreover, by Proposition 4.17, the constraint $\mu(z)$ is given by two linear functions on $I$ :

$$
\mu(z)= \begin{cases}\alpha+\beta z & \text { if } z \leqslant s^{\prime}, z \in I, \\ \alpha^{\prime}+\beta^{\prime} z & \text { if } z \geqslant s^{\prime}, z \in I .\end{cases}
$$

Thus, $s^{\prime}$ is the only non-smooth point of $\mu$ on $I$. But since $s_{0} \in S_{+}$and $\mu \leqslant c, s_{0}$ is also a non-smooth point of $\mu$, and so $s^{\prime}=s_{0}$. Now, since $c$ is nondecreasing and by 6.10 , we see that $\beta^{\prime} \geqslant 0$.

Let $k \geqslant 1$ be such that $s_{0} \in\left[c_{2 k+1}, c_{2 k-1}\right]$. Since $c_{2 k+1}$ and $c_{2 k-1}$ are regular by assumption, we have $\left.s_{0} \in\right] c_{2 k+1}, c_{2 k-1}\left[\right.$. Notice that $u_{k}(j) \rightarrow c_{2 k+1}$ and $v_{k}(j) \rightarrow c_{2 k-1}$ as $j \rightarrow \infty$. Let $u_{-}, u_{+}$be the two points from the sequence

$$
c_{2 k+1}<\cdots<u_{k}(2)<u_{k}(1)=v_{k}(6)<v_{k}(7)<\cdots<c_{2 k-1}
$$

of Corollary 6.5 (ii) such that $s_{0} \in\left[u_{-}, u_{+}\right]$. Since $u_{-}$and $u_{+}$are regular by assumption, we must have $\left.s_{0} \in\right] u_{-}, u_{+}[$. But then Lemma 6.9 shows that $\mu\left(s_{0}\right)>\frac{s_{0}+1}{4}>\frac{u_{-}+1}{4}=\varphi\left(u_{-}\right)>\psi\left(u_{+}\right)=\sqrt{\frac{u_{+}}{2}}$. And since $\beta^{\prime} \geqslant 0$, we find 
that $\mu\left(u_{+}\right) \geqslant \mu\left(s_{0}\right)>\sqrt{\frac{u_{+}}{2}}$, and thus $u_{+} \in I$, and so $l\left(u_{+}\right)>l\left(s_{0}\right)$. But, for all $z \in] u_{-}, u_{+}\left[\right.$we have $l(z)>l\left(u_{-}\right)$and $l(z)>l\left(u_{+}\right)$. In particular, $l\left(s_{0}\right)>$ $l\left(u_{+}\right)$, which is a contradiction.

Lemma 6.12. The points $u_{k}(j)$ with $k \geqslant 1, j \geqslant 2$ are regular.

Proof. Abbreviate $u:=u_{k}(j)=: \frac{p}{q}$. Let us first prove that a class of the form $\left(d+\frac{1}{2}, d-\frac{1}{2} ; m\right)$ with $l(m)=l(u)$ cannot give an obstruction bigger than $\frac{u+1}{4}$. Suppose by contradiction that there is such a class $\left(d+\frac{1}{2}, d-\frac{1}{2} ; m\right)$. By Proposition 6.2(i) and Corollary 6.8(i),

$$
\frac{\sqrt{2} d}{q \sqrt{u}}<\frac{2}{q \sqrt{u^{2}-6 u+1}}=\frac{2}{\sqrt{j^{2}+6 j+1}}
$$

which is smaller than 1 for all $j \geqslant 2$. Since $l(m)=l(u), m_{i} \geqslant 1$ for all $i$. Thus

$$
\sum \varepsilon_{i}^{2} \geqslant j\left(1-\frac{\sqrt{2} d}{q \sqrt{u}}\right)^{2}>j\left(1-\frac{2}{\sqrt{j^{2}+6 j+1}}\right)^{2}=: s(j) .
$$

Now, since $s(j)$ is increasing for $j \geqslant 2$ and $s(2)>\frac{1}{2}$, we have $\mu\left(d+\frac{1}{2}, d-\right.$ $\left.\frac{1}{2} ; m\right)(u) \leqslant \sqrt{\frac{u}{2}}<\frac{u+1}{4}$ by Lemma 4.8 (iii). The lemma is thus proven for a class of the form $\left(d+\frac{1}{2}, d-\frac{1}{2} ; m\right)$.

Let us now prove it for a class of the form $(d, d ; m)$. Suppose that there exists a class $(d, d ; m) \in \mathcal{E}$ with $l(m)=l(u)$ such that $\mu(d, d ; m)(u)>\frac{u+1}{4}$. By Proposition 6.2(i) and Corollary 6.8(i),

$$
\frac{\sqrt{2} d}{q \sqrt{u}}<\frac{2 \sqrt{2}}{q \sqrt{u^{2}-6 u+1}}=\frac{2 \sqrt{2}}{\sqrt{j^{2}+6 j+1}}
$$

which is smaller than 1 for all $j \geqslant 2$. Since $l(m)=l(u), m_{i} \geqslant 1$ for all $i$. Thus

$$
\sum \varepsilon_{i}^{2} \geqslant j\left(1-\frac{\sqrt{2} d}{q \sqrt{u}}\right)^{2}>j\left(1-\frac{2 \sqrt{2}}{\sqrt{j^{2}+6 j+1}}\right)^{2}=: s(j) .
$$

Now, since $s(j)$ is increasing for $j \geqslant 2$ and $s(4)>1$, we have $\mu(d, d ; m)(u) \leqslant$ $\sqrt{\frac{u}{2}}<\frac{u+1}{4}$ by Lemma 4.8 (iii). So the lemma is proven for $j \geqslant 4$. 
It remains to show the lemma for $j=2,3$. By Proposition 6.2 (ii),

$$
\sum \varepsilon_{i}^{2}=1-\lambda^{2}<1-d^{2} \sqrt{\frac{2}{u}} y(u)
$$

Thus

$$
\begin{aligned}
0 & =\sum \varepsilon_{i}^{2}+\lambda^{2}-1>j\left(1-\frac{\sqrt{2} d}{q \sqrt{u}}\right)^{2}+d^{2} \sqrt{\frac{2}{u}} y(u)-1 \\
& =\left(\frac{2 j}{q^{2} u}+\sqrt{\frac{2}{u}} y(u)\right) d^{2}-\frac{2 \sqrt{2} j}{q \sqrt{u}} d+j-1=: f(d) .
\end{aligned}
$$

To obtain a contradiction, we need to show that $f(d) \geqslant 0$ for all $d \geqslant 1$. Since $y(u)>0$ for $u>\sigma^{2}$, it is sufficient to show that the discriminant of $f(d)$ is negative, that is:

$$
\frac{8 j^{2}}{q^{2} u}-4(j-1)\left(\frac{2 j}{q^{2} u}+\sqrt{\frac{2}{u}} y(u)\right) \leqslant 0,
$$

which is equivalent to

$$
\frac{j}{q^{2}}+2(j-1) u \leqslant(j-1) \sqrt{\frac{u}{2}}(u+1) .
$$

Taking squares and using $u=\frac{p}{q}$, we get

(6.11) $0 \leqslant-2 j^{2}+p q\left((j-1)^{2} p^{2}-6(j-1)^{2} p q+(j-1)^{2} q^{2}-8 j(j-1)\right)$.

For $j=2$, this gives

$$
0 \leqslant-8+p q\left(p^{2}-6 p q+q^{2}-16\right)
$$

and this inequality is true since $p q \geqslant 26$ and $p^{2}-6 p q+q^{2}-16=1$ by Corollary 6.8 (iii). For $j=3$, we get

$$
0 \leqslant-9+p q\left(2 p^{2}-12 p q+2 q^{2}-24\right)
$$

and this inequality is also true since $p q \geqslant 57$ and $2 p^{2}-12 p q+2 q^{2}-24=32$ by Corollary 6.8(iii). This concludes the proof.

Lemma 6.13. The points $v_{k}(j)$ with $k \geqslant 1, j \geqslant 6$ are regular. 
Proof. Abbreviate $v:=v_{k}(j)=: \frac{p}{q}$. Let us first prove that a class of the form $\left(d+\frac{1}{2}, d-\frac{1}{2} ; m\right)$ with $l(m)=l(v)$ cannot give an obstruction bigger than $\frac{v+1}{4}$. Suppose by contradiction that there is such a class $\left(d+\frac{1}{2}, d-\frac{1}{2} ; m\right)$. By Proposition 6.2(i) and Corollary 6.8(ii),

$$
\frac{\sqrt{2} d}{q \sqrt{u}}<\frac{2}{q \sqrt{v^{2}-6 v+1}}=\frac{2}{\sqrt{j^{2}-4 j-4}}
$$

which is smaller than 1 for all $j \geqslant 6$. Since $l(m)=l(v), m_{i} \geqslant 1$ for all $i$. Thus

$$
\sum \varepsilon_{i}^{2} \geqslant j\left(1-\frac{\sqrt{2} d}{q \sqrt{u}}\right)^{2}>j\left(1-\frac{2}{\sqrt{j^{2}-4 j-4}}\right)^{2}=: s(j) .
$$

Now, since $s(j)$ is increasing for $j \geqslant 6$ and $s(6)>\frac{1}{2}$, we have $\mu\left(d+\frac{1}{2}, d-\right.$ $\left.\frac{1}{2} ; m\right)(v) \leqslant \sqrt{\frac{v}{2}}<\frac{v+1}{4}$ by Lemma 4.8 (iii). The lemma is thus proven for a class of the form $\left(d+\frac{1}{2}, d-\frac{1}{2} ; m\right)$.

Let us now prove the lemma for a class of the form $(d, d ; m)$. Suppose that there exists a class $(d, d ; m) \in \mathcal{E}$ with $l(m)=l(v)$ such that $\mu(d, d ; m)(v)>$ $\frac{v+1}{4}$. By Proposition 6.2(i) and Corollary 6.8(ii),

$$
\frac{\sqrt{2} d}{q \sqrt{u}}<\frac{2 \sqrt{2}}{q \sqrt{v^{2}-6 v+1}}=\frac{2 \sqrt{2}}{\sqrt{j^{2}-4 j-4}}
$$

which is smaller than 1 for all $j \geqslant 6$. Since $l(m)=l(u), m_{i} \geqslant 1$ for all $i$. Thus

$$
\sum \varepsilon_{i}^{2} \geqslant j\left(1-\frac{\sqrt{2} d}{q \sqrt{u}}\right)^{2}>j\left(1-\frac{2 \sqrt{2}}{\sqrt{j^{2}-4 j-4}}\right)^{2}=: s(j) .
$$

Now, since $s(j)$ is increasing for $j \geqslant 6$ and $s(8)>1$, we have $\mu(d, d ; m)(v) \leqslant$ $\sqrt{\frac{v}{2}}<\frac{v+1}{4}$ by Lemma 4.8 (iii). So the lemma is proven for $j \geqslant 8$.

It remains to show it for $j=6,7$. If $j=6$, the same arguments as in the proof of Lemma 6.12 in the cases $j=2,3$ show that a point $v_{k}(6)=: \frac{p}{q}$ is regular if 6.11 is satisfied for $j=6$, that is if and only if

$$
0 \leqslant-72+p q\left(25 p^{2}-150 p q+25 q^{2}-160\right) .
$$

This inequality is true since $p q \geqslant 287$ and $25 p^{2}-150 p q+25 q^{2}-160=40$ by Corollary 6.8(iv). Similarly, a point $v_{k}(7)=: \frac{p}{q}$ is regular if 6.11) is satisfied for $j=7$, that is if and only if 


$$
0 \leqslant-98+p q\left(36 p^{2}-216 p q+36 q^{2}-336\right)
$$

which is true since $p q \geqslant 376$ and $36 p^{2}-216 p q+36 q^{2}-336=276$ by Corollary 6.8 (iv). This completes the proof.

Definition 6.14. Define for $k \geqslant 1$ and $i \geqslant 0$ the points

$$
b_{k}(i):=v_{k}(2+2 i)=\left[5 ;\{1,4\}^{\times(k-1)}, 1,2+2 i\right] .
$$

In particular, for all $k \geqslant 1, u_{k}(1)=v_{k}(6)=b_{k}(2)$. Let $\frac{p}{q}:=b_{k}(i)$ written in lowest terms. We will now associate to every $b_{k}(i)$ a class $E\left(b_{k}(i)\right) \in \mathcal{E}$ for which we will prove that it gives the constraint at $b_{k}(i)$. We distinguish the cases $i$ even and $i$ odd.

If $i=2 j$, set

$$
m_{k}(2 j):=q w\left(b_{k}(2 j)\right)
$$

but with the last block $\left(1^{\times(4 j+2)}\right)$ being replaced by the block

$$
\left(j+1, j, 1^{\times(2 j+1)}\right),
$$

and $d_{k}(2 j):=q \frac{1+b_{k}(2 j)}{4}=\frac{1}{4}(p+q)$. Then define the class

$$
E\left(b_{k}(2 j)\right):=\left(d_{k}(2 j), d_{k}(2 j) ; m_{k}(2 j)\right) .
$$

If $i=2 j+1$, set

$$
m_{k}(2 j+1):=q w\left(b_{k}(2 j+1)\right)
$$

but with the last block $\left(1^{\times(4 j+4)}\right)$ being replaced by the block

$$
\left((j+1)^{\times 2}, 1^{\times(2 j+2)}\right),
$$

and $d_{k}(2 j+1):=q \frac{1+b_{k}(2 j+1)}{4}=\frac{1}{4}(p+q)$. Then define the class

$$
E\left(b_{k}(2 j+1)\right):=\left(d_{k}(2 j+1)+\frac{1}{2}, d_{k}(2 j+1)-\frac{1}{2} ; m_{k}(2 j+1)\right) .
$$

We will now prove that the classes $E\left(b_{k}(i)\right)$ belong to $\mathcal{E}$.

Lemma 6.15. For all $k \geqslant 1, i \geqslant 0$ the classes $E\left(b_{k}(i)\right)$ satisfy the Diophantine conditions of Proposition 3.8 (i). 
Proof. We first treat the case $i=2 j$. Abbreviate $b:=b_{k}(2 j)=: \frac{p}{q}, m:=m_{k}(2 j)$, $w:=w\left(b_{k}(2 j)\right)$ and $d:=d_{k}(2 j)$. By Lemma 2.5(iii),

$$
\sum_{l} m_{l}=q \sum_{l} w_{l}=q\left(b+1-\frac{1}{q}\right)=4\left(q \frac{1+b}{4}\right)-1=4 d-1,
$$

which proves that the first equation holds. For the second equation, we have

$$
\sum_{l} m_{l}^{2}=q^{2}\left(\sum_{l} w_{l}^{2}\right)-2 j-1+(j+1)^{2}+j^{2}=q^{2} b+2 j^{2}=p q+2 j^{2} .
$$

On the other hand,

$$
2 d^{2}+1=2\left(q^{2} \frac{(1+b)^{2}}{16}\right)+1=\frac{1}{8}(p+q)^{2}+1 .
$$

By Lemma 6.6 it suffices that this equals $p q+2 j^{2}$ for three small values of $j$, which is the case.

Similarly in the case $i=2 j+1$, abbreviate $b:=b_{k}(2 j+1)=: \frac{p}{q}, m:=$ $m_{k}(2 j+1), w:=w\left(b_{k}(2 j+1)\right)$ and $d:=d_{k}(2 j+1)$. We then have

$$
\sum_{l} m_{l}=q \sum_{l} w_{l}=q\left(b+1-\frac{1}{q}\right)=4\left(q \frac{1+b}{4}\right)-1=4 d-1 .
$$

For the second equation we have

$$
\sum_{l} m_{l}^{2}=q^{2}\left(\sum_{l} w_{l}^{2}\right)-2 j-2+2(j+1)^{2}=q^{2} b+2 j^{2}+2 j=p q+2 j^{2}+2 j .
$$

On the other hand,

$$
2 d^{2}+\frac{1}{2}=2\left(q^{2} \frac{(1+b)^{2}}{16}\right)+\frac{1}{2}=\frac{1}{8}(p+q)^{2}+\frac{1}{2} .
$$

Now use again Lemma 6.6 to check that this equals $p q+2 j^{2}+2 j$ for all $j \geqslant 0$.

Lemma 6.16. The classes $E\left(b_{k}(2 j)\right)$ reduce to $(0 ;-1)$ for all $k \geqslant 1, j \geqslant 0$.

Proof. The proof is by induction over $k$. For the initial step $k=1$, we have $b_{1}(2 j)=\frac{24 j+17}{4 j+3}=: \frac{p}{q}, q w\left(b_{1}(2 j)\right)=\left((4 j+3)^{\times 5}, 4 j+2,1^{\times(4 j+2)}\right)$, and $d_{1}(2 j)$ 
$=7 j+5$. Thus

$$
E\left(b_{1}(2 j)\right)=\left(7 j+5,7 j+5 ;(4 j+3)^{\times 5}, 4 j+2, j+1, j, 1^{\times(2 j+1)}\right) .
$$

We now show that this class reduces to $(0 ;-1)$.

$$
\begin{aligned}
& \left(7 j+5,7 j+5 ;(4 j+3)^{\times 5}, 4 j+2, j+1, j, 1^{\times(2 j+1)}\right) \stackrel{\varphi_{*}}{\longmapsto} \\
& \left(10 j+7 ;(4 j+3)^{\times 4}, 4 j+2,(3 j+2)^{\times 2}, j+1, j, 1^{\times(2 j+1)}\right) ; \\
& \left(8 j+5 ; 4 j+3,4 j+2,(3 j+2)^{\times 2},(2 j+1)^{\times 3}, j+1, j, 1^{\times(2 j+1)}\right) ; \\
& \left(5 j+3 ; 3 j+2,(2 j+1)^{\times 3},(j+1)^{\times 2}, j^{\times 2}, 1^{\times(2 j+1)}\right) ; \\
& \left(3 j+2 ; 2 j+1,(j+1)^{\times 3}, j^{\times 2}, 1^{\times(2 j+1)}\right) ; \\
& \left(2 j+1 ; j+1, j^{\times 3}, 1^{\times(2 j+1)}\right) ; \\
& \left(j+1 ; j, 1^{\times(2 j+2)}\right) .
\end{aligned}
$$

This class reduces to $(0 ;-1)$ in $j+1$ steps since a class of the type $\left(s+1 ; s, 1^{\times t}\right)$ for $s \geqslant 1, t \geqslant 2$ reduces to $\left(s ; s-1,1^{\times(t-2)}\right)$ by a standard Cremona move.

We turn now to the general case. We will freely use the definitions of the Pell numbers $P_{n}$ and the Half companion Pell numbers $H_{n}$ given in Definition 6.3 and the fact that for all $n \geqslant 0, H_{n}=P_{n}+P_{n-1}$. Suppose that the class $E\left(b_{k-1}(2 j)\right)$ reduces to $(0 ;-1)$ and let us show that the class $E\left(b_{k}(2 j)\right)$ also reduces to $(0 ;-1)$. We have

$$
\begin{aligned}
& b_{k}(2 j)=\frac{2 j P_{2 k+2}+H_{2 k+2}}{2 j P_{2 k}+H_{2 k}}, \\
& d_{k}(2 j)=j H_{2 k+1}+P_{2 k+1} .
\end{aligned}
$$

The first terms of the class $E\left(b_{k}(2 j)\right)$ are

$$
\begin{aligned}
& \left(j H_{2 k+1}+P_{2 k+1}, j H_{2 k+1}+P_{2 k+1} ;\left(2 j P_{2 k}+H_{2 k}\right)^{\times 5}, 4 j P_{2 k-1}+H_{2 k-1},\right. \\
& \left.\left(2 j P_{2 k-2}+H_{2 k-2}\right)^{\times 4}, 4 j P_{2 k-3}+2 H_{2 k-3},(*)\right),
\end{aligned}
$$

where $(*)$ stands for all the next terms. The image of $E\left(b_{k}(2 j)\right)$ under $\varphi_{*}$ is

$$
\begin{aligned}
& \left(2 j P_{2 k+1}+H_{2 k+1} ;\left(2 j P_{2 k}+H_{2 k}\right)^{\times 4}, 4 j P_{2 k-1}+2 H_{2 k-1},\left(j H_{2 k}+P_{2 k}\right)^{\times 2}\right. \\
& \left.\quad\left(2 j P_{2 k-2}+H_{2 k-2}\right)^{\times 4}, 4 j P_{2 k-3}+2 H_{2 k-3},(*)\right) .
\end{aligned}
$$

To finish the proof, we will show that $\varphi_{*}\left(E\left(b_{k}(2 j)\right)\right)$ reduces to the class $\varphi_{*}\left(E\left(b_{k-1}(2 j)\right)\right)$ in four steps. 


$$
\begin{aligned}
& \left(2 j P_{2 k+1}+H_{2 k+1} ;\left(2 j P_{2 k}+H_{2 k}\right)^{\times 4}, 4 j P_{2 k-1}+2 H_{2 k-1},\right. \\
& \left.\quad\left(j H_{2 k}+P_{2 k}\right)^{\times 2},\left(2 j P_{2 k-2}+H_{2 k-2}\right)^{\times 4}, 4 j P_{2 k-3}+2 H_{2 k-3},(*)\right) ; \\
& \left(2 j\left(H_{2 k}+P_{2 k-1}\right)+3 H_{2 k-1}+2 P_{2 k-1} ; 2 j P_{2 k}+H_{2 k}, 4 j P_{2 k-1}+2 H_{2 k-1},\right. \\
& \quad\left(j H_{2 k}+P_{2 k}\right)^{\times 2},\left(2 j P_{2 k-1}+H_{2 k-1}\right)^{\times 3},\left(2 j P_{2 k-2}+H_{2 k-2}\right)^{\times 4}, \\
& \left.\quad 4 j P_{2 k-3}+2 H_{2 k-3},(*)\right) ; \\
& \left(j\left(3 H_{2 k}-2 P_{2 k}\right)+2 H_{2 k-1}+P_{2 k-1} ; j H_{2 k}+P_{2 k},\left(2 j P_{2 k-1}+H_{2 k-1}\right)^{\times 3},\right. \\
& \quad j H_{2 k-1}+P_{2 k-1},\left(2 j P_{2 k-2}+H_{2 k-2}\right)^{\times 4}, 4 j P_{2 k-3}+2 H_{2 k-3}, \\
& \left.\quad j H_{2 k-2}+P_{2 k-2},(*)\right) ; \\
& \left(j H_{2 k}+P_{2 k} ; 2 j P_{2 k-1}+H_{2 k-1},\left(j H_{2 k-1}+P_{2 k-1}\right)^{\times 2},\right. \\
& \left.\quad\left(2 j P_{2 k-2}+H_{2 k-2}\right)^{\times 4}, 4 j P_{2 k-3}+2 H_{2 k-3}, j H_{2 k-2}+P_{2 k-2},(*)\right) ; \\
& \left(2 j P_{2 k-1}+H_{2 k-1} ;\left(2 j P_{2 k-2}+H_{2 k-2}\right)^{\times 4}, 4 j P_{2 k-3}+2 H_{2 k-3},\right. \\
& \left.\quad\left(j H_{2 k-2}+P_{2 k-2}\right)^{\times 2},(*)\right) .
\end{aligned}
$$

It is important to note that $(*)$ was left invariant during the whole reduction process. So the last class is precisely $\varphi_{*}\left(E\left(b_{k-1}(2 j)\right)\right)$.

Lemma 6.17. The classes $E\left(b_{k}(2 j+1)\right)$ reduce to $(0 ;-1)$ for all $k \geqslant 1$, $j \geqslant 0$.

Proof. The proof is again by induction over $k$. For $k=1$, we have that $b_{1}(2 j+$ $1)=\frac{24 j+29}{4 j+5}=: \frac{p}{q}, q w\left(b_{1}(2 j+1)\right)=\left((4 j+5)^{\times 5}, 4 j+4,1^{\times(4 j+4)}\right), d_{1}(2 j+1)$ $=7 j+\frac{17}{2}$. Thus

$$
E\left(b_{1}(2 j+1)\right)=\left(7 j+9,7 j+8 ;(4 j+5)^{\times 5}, 4 j+4,(j+1)^{2}, 1^{\times(2 j+2)}\right) .
$$

We show now that this class reduces to $(0 ;-1)$.

$$
\begin{aligned}
& \left(7 j+9,7 j+8 ;(4 j+5)^{\times 5}, 4 j+4,(j+1)^{2}, 1^{\times(2 j+2)}\right) \stackrel{\varphi_{*}}{\longmapsto} \\
& \left(10 j+2 ;(4 j+5)^{\times 4}, 4 j+4,3 j+4,3 j+3,(j+1)^{2}, 1^{\times(2 j+2)}\right) ; \\
& \left(8 j+9 ; 4 j+5,4 j+4,3 j+4,3 j+3,(2 j+2)^{\times 3},(j+1)^{2}, 1^{\times(2 j+2)}\right) ; \\
& \left(5 j+5 ; 3 j+3,(2 j+2)^{\times 3},(j+1)^{\times 3}, j, 1^{\times(2 j+2)}\right) ; \\
& \left(3 j+3 ; 2 j+2,(j+1)^{\times 4}, j, 1^{\times(2 j+2)}\right) ;
\end{aligned}
$$




$$
\begin{aligned}
& \left(2 j+2 ;(j+1)^{\times 3}, j, 1^{\times(2 j+2)}\right) ; \\
& \left(j+1 ; j, 1^{\times(2 j+2)}\right) .
\end{aligned}
$$

As seen before, this class reduces to $(0 ;-1)$ in $j+1$ steps.

We turn now to the general case. Suppose that the class $E\left(b_{k-1}(2 j+1)\right)$ reduces to $(0 ;-1)$ and let us show that the class $E\left(b_{k}(2 j+1)\right)$ also reduces to $(0 ;-1)$. We have

$$
\begin{aligned}
& b_{k}(2 j+1)=\frac{2 j P_{2 k+2}+P_{2 k+3}}{2 j P_{2 k}+P_{2 k+1}}, \\
& d_{k}(2 j+1)=j H_{2 k+1}+\frac{1}{2} H_{2 k+2} .
\end{aligned}
$$

The first terms of the class $E\left(b_{k}(2 j+1)\right)$ are

$$
\begin{gathered}
\left(j H_{2 k+1}+\frac{1}{2} H_{2 k+2}+\frac{1}{2}, j H_{2 k+1}+\frac{1}{2} H_{2 k+2}-\frac{1}{2} ;\left(2 j P_{2 k}+P_{2 k+1}\right)^{\times 5},\right. \\
\left.4 j P_{2 k-1}+2 P_{2 k},\left(2 j P_{2 k-2}+P_{2 k-1}\right)^{\times 4}, 4 j P_{2 k-3}+2 P_{2 k-2},(*)\right) .
\end{gathered}
$$

The image of $E\left(b_{k}(2 j+1)\right)$ under $\varphi_{*}$ is

$$
\begin{aligned}
& \left(2 j P_{2 k+1}+P_{2 k+2} ;\left(2 j P_{2 k}+P_{2 k+1}\right)^{\times 4}, 4 j P_{2 k-1}+2 P_{2 k},\right. \\
& \quad j H_{2 k}+\frac{1}{2} H_{2 k+1}+\frac{1}{2}, j H_{2 k}+\frac{1}{2} H_{2 k+1}-\frac{1}{2},\left(2 j P_{2 k-2}+P_{2 k-1}\right)^{\times 4}, \\
& \left.\quad 4 j P_{2 k-3}+2 P_{2 k-2},(*)\right),
\end{aligned}
$$

To finish the proof, we will show that $\varphi_{*}\left(E\left(b_{k}(2 j+1)\right)\right)$ reduces to the vector $\varphi_{*}\left(E\left(b_{k-1}(2 j+1)\right)\right)$ in four steps.

$$
\begin{aligned}
& \left(2 j P_{2 k+1}+P_{2 k+2} ;\left(2 j P_{2 k}+P_{2 k+1}\right)^{\times 4}, 4 j P_{2 k-1}+2 P_{2 k},\right. \\
& \quad j H_{2 k}+\frac{1}{2} H_{2 k+1}+\frac{1}{2}, j H_{2 k}+\frac{1}{2} H_{2 k+1}-\frac{1}{2},\left(2 j P_{2 k-2}+P_{2 k-1}\right)^{\times 4}, \\
& \left.\quad 4 j P_{2 k-3}+2 P_{2 k-2},(*)\right) ; \\
& \left(j\left(2 P_{2 k}+4 P_{2 k-1}\right)+\left(P_{2 k+1}+2 P_{2 k}\right) ; 2 j P_{2 k}+P_{2 k+1}, 4 j P_{2 k-1}+2 P_{2 k},\right. \\
& \quad j H_{2 k}+\frac{1}{2} H_{2 k+1}+\frac{1}{2}, j H_{2 k}+\frac{1}{2} H_{2 k+1}-\frac{1}{2},\left(2 j P_{2 k-1}+P_{2 k}\right)^{\times 3}, \\
& \left.\quad\left(2 j P_{2 k-2}+P_{2 k-1}\right)^{\times 4}, 4 j P_{2 k-3}+2 P_{2 k-2},(*)\right) ; \\
& \left(j\left(P_{2 k}+3 P_{2 k-1}\right)+\left(\frac{1}{2} P_{2 k+1}+\frac{3}{2} P_{2 k}-\frac{1}{2}\right) ; j H_{2 k}+\frac{1}{2} H_{2 k+1}-\frac{1}{2},\right. \\
& \quad\left(2 j P_{2 k-1}+P_{2 k}\right)^{\times 3}, j H_{2 k-1}+\frac{1}{2} H_{2 k}-\frac{1}{2},\left(2 j P_{2 k-2}+P_{2 k-1}\right)^{\times 4}, \\
& \left.\quad 4 j P_{2 k-3}+2 P_{2 k-2}, j H_{2 k-2}+\frac{1}{2} H_{2 k-1}-\frac{1}{2},(*)\right) ; \\
& \left(j H_{2 k}+\frac{1}{2} H_{2 k+1}-\frac{1}{2} ; 2 j P_{2 k-1}+P_{2 k},\left(j H_{2 k-1}+\frac{1}{2} H_{2 k}-\frac{1}{2}\right)^{\times 2},\right. \\
& \left.\quad\left(2 j P_{2 k-2}+P_{2 k-1}\right)^{\times 4}, 4 j P_{2 k-3}+2 P_{2 k-2}, j H_{2 k-2}+\frac{1}{2} H_{2 k-1}-\frac{1}{2},(*)\right) ;
\end{aligned}
$$




$$
\begin{gathered}
\left(2 j P_{2 k-1}-P_{2 k} ;\left(2 j P_{2 k-2}+P_{2 k-1}\right)^{\times 4}, 4 j P_{2 k-3}+2 P_{2 k-2},\right. \\
\left.j H_{2 k-2}+\frac{1}{2} H_{2 k-1}+\frac{1}{2}, j H_{2 k-2}+\frac{1}{2} H_{2 k-1}-\frac{1}{2},(*)\right) .
\end{gathered}
$$

Since $(*)$ was left invariant during the whole reduction process, the last class is precisely $\varphi_{*}\left(E\left(b_{k-1}(2 j+1)\right)\right)$.

Proposition 6.18. For all $k \geqslant 1, i \geqslant 0$, we have $E\left(b_{k}(i)\right) \in \mathcal{E}$.

Proof. We have to show that the classes $E\left(b_{k}(i)\right)$ satisfy the Diophantine conditions of Proposition 3.8, which we have done in Lemma 6.15, and that they reduce to $(0 ;-1)$ by Cremona moves, which we have done for $i$ even in Lemma 6.16 and for $i$ odd in Lemma 6.17. The proof is thus complete.

Corollary 6.19. For all $n \geqslant 0$, the classes $E\left(\beta_{n}\right)$ of Theorem 5.1 belong to $\mathcal{E}$.

Proof. Notice that by Lemma 6.4, for all $k \geqslant 0$,

$$
\begin{aligned}
& b_{k}(0)=v_{k}(2)=\frac{P_{2 k+2}+P_{2 k+1}}{P_{2 k}+P_{2 k-1}}=\frac{H_{2 k+2}}{H_{2 k}}=\beta_{2 k}, \\
& b_{k}(1)=v_{k}(4)=\frac{2 P_{2 k+2}+P_{2 k+1}}{2 P_{2 k}+P_{2 k-1}}=\frac{P_{2 k+3}}{P_{2 k+1}}=\beta_{2 k+1} .
\end{aligned}
$$

Hence by Definition 6.14, we see that for all $k \geqslant 0, E\left(b_{k}(0)\right)=E\left(\beta_{2 k}\right)$ and $E\left(b_{k}(1)\right)=E\left(\beta_{2 k+1}\right)$. Thus all the classes $E\left(\beta_{n}\right)$ belong indeed to $\mathcal{E}$.

Corollary 6.20. $c\left(b_{k}(i)\right)=\frac{b_{k}(i)+1}{4}$ for all $k \geqslant 1$ and $i \geqslant 2$.

Proof. Since $\left.b_{k}(i) \in\right] \sigma^{2}, 6\left[\right.$, we can write them as $b_{k}(i)=5+x$ where $x \in$ $\left[0,1\left[\right.\right.$. Now, $\left(2,2 ; 2,1^{\times 5}\right) \in \mathcal{E}$, thus

$$
\mu\left(2,2 ; 2,1^{\times 5}\right)\left(b_{k}(i)\right)=\frac{6+x}{4}=\frac{b_{k}(i)+1}{4} .
$$

So $c\left(b_{k}(i)\right) \geqslant \mu\left(2,2 ; 2,1^{\times 5}\right)\left(b_{k}(i)\right)=\frac{b_{k}(i)+1}{4}$.

Let us show the converse inequality. Abbreviate $b:=b_{k}(i)=: \frac{p}{q}$ in lowest terms, $d:=d_{k}(i)$ and $m:=m_{k}(i)$. Then

$$
\mu(E(b))(b)=\frac{\langle m, w(b)\rangle}{2 d}=\frac{\langle q w(b), w(b)\rangle}{2 d}=\frac{2 b}{1+b}<\sqrt{\frac{b}{2}}<\frac{b+1}{4}
$$

since $b>\sigma^{2}$. Now if $\left(d^{\prime}, e^{\prime} ; m^{\prime}\right) \in \mathcal{E}$ is a class different from $E(b)$, we have by Proposition 3.8 (ii) that $\left\langle m, m^{\prime}\right\rangle \leqslant d\left(d^{\prime}+e^{\prime}\right)$. Using the definitions of $d$ and 
$m$ and the fact that $m$ is written in decreasing order, we get

$$
q \frac{1+b}{4}\left(d^{\prime}+e^{\prime}\right) \geqslant q\left\langle w(b), m^{\prime}\right\rangle
$$

thus

$$
\mu\left(d^{\prime}, e^{\prime} ; m^{\prime}\right)(b)=\frac{\left\langle m^{\prime}, w(b)\right\rangle}{d^{\prime}+e^{\prime}} \leqslant \frac{b+1}{4} .
$$

The proof is complete.

Theorem 6.21. $c(a)=\frac{a+1}{4}$ on $\left[\sigma^{2}, 6\right]$.

Proof. By Proposition 6.11 it suffices to show that the points $c_{2 k-1}$ for all $k \geqslant 1, u_{k}(j)$ for all $k \geqslant 1, j \geqslant 2$, and $v_{k}(j)$ for all $k \geqslant 1, j \geqslant 6$ are regular. By Lemma 6.12 and Lemma 6.13 the points $u_{k}(j)$ and $v_{k}(j)$ are regular. Moreover, for all $k \geqslant 1, v_{k}(j) \underset{j \rightarrow \infty}{\longrightarrow} c_{2 k-1}$. But by Corollary 6.20, $c\left(v_{k}(2+2 i)\right)=$ $c\left(b_{k}(i)\right)=\frac{b_{k}(i)+1}{4}$ for $i \geqslant 2$. So, by continuity of $c$, we get that $c\left(c_{2 k-1}\right)=$ $\frac{c_{2 k-1}+1}{4}$, and the points $c_{2 k-1}$ are thus regular. This completes the proof.

\section{The interval $[6,8]$}

\subsection{Preliminaries}

We will use the fact that if $\left[l_{0} ; l_{1}, \ldots, l_{N}\right]$ is a continued fraction of a rational number $\frac{p}{q}$ and $\frac{p_{k}}{q_{k}}:=\left[l_{0} ; l_{1}, \ldots, l_{k}\right]$ is its $k$-th convergent written in lowest terms, then for any real number $x$,

$$
\left[l_{0} ; l_{1}, \ldots, l_{k}, x\right]=\frac{x p_{k}+p_{k-1}}{x q_{k}+q_{k-1}}
$$

written in lowest terms. In particular, $q_{k}=l_{k} q_{k-1}+q_{k-2}$. It is then easy to see that if $L:=\sum_{i} l_{i}$, then

$$
q=q_{N} \geqslant L
$$

Recall also that we defined the error vector of a class $(d, e ; m)$ at a point $a$ as the vector $\varepsilon:=\varepsilon((d, e ; m), a)$ defined by the equation

$$
m=\frac{d+e}{\sqrt{2 a}} w(a)+\varepsilon .
$$


Now set then $M:=l(a)=L+l_{0}$,

$$
\sigma:=\sum_{i>l_{0}} \varepsilon_{i}^{2}
$$

and

$$
\sigma^{\prime}:=\sum_{i=l_{0}+1}^{M-l_{N}} \varepsilon_{i}^{2}<\sigma .
$$

Then by Lemma 4.8.3, $\sigma<1$ for a class of the form $(d, d ; m)$ and $\sigma<\frac{1}{2}$ for a class of the form $\left(d+\frac{1}{2}, d-\frac{1}{2} ; m\right)$.

Lemma 7.1. Let $(d, e ; m) \in \mathcal{E}$ be a class such that there exists $a=: \frac{p}{q} \in$ ]$\sigma^{2}, 8[$ with $l(a)=l(m)$ and

$$
\mu(d, e ; m)(a)>\sqrt{\frac{a}{2}} .
$$

Assume that $y(a):=a+1-2 \sqrt{2 a}>\frac{1}{q}$, and set $v_{M}:=\frac{d+e}{q \sqrt{2 a}}$. Then

(i) $\left|\sum \varepsilon_{i}\right| \leqslant \sqrt{\sigma L}$,

(ii) If $v_{M}<1$, then $\left|\sum \varepsilon_{i}\right| \leqslant \sqrt{\sigma^{\prime} L}$,

(iii) If $v_{M} \leqslant \frac{1}{2}$, then $v_{M}>\frac{1}{3}$ and $\sigma^{\prime} \leqslant \frac{1}{2}$. If $v_{M} \leqslant \frac{3}{4}$, then $\sigma^{\prime} \leqslant \frac{7}{8}$,

(iv) Set $\delta:=y(a)-\frac{1}{q}>0$. Then for both types of classes $(d, d ; m)$ and $(d+$ $\left.\frac{1}{2}, d-\frac{1}{2} ; m\right)$ we have

$$
d \leqslant \frac{\sqrt{a}}{\sqrt{2} \delta}(\sqrt{\sigma L}-1) \leqslant \frac{\sqrt{a}}{\sqrt{2} \delta}(\sqrt{\sigma q}-1)<\frac{\sqrt{a}}{\sqrt{2} \delta}\left(\frac{\sigma}{\delta v_{M}}-1\right) .
$$

If $v_{M}<1, \sigma$ can be replaced by $\sigma^{\prime}$.

Proof. The proofs of (i), (ii) and (iii) are the same as in the proof of Lemma 5.1.2 in [MS]. To prove (iv), we notice first that $\sum \varepsilon_{i}<0$. Indeed, by Lemma $4.84,-\sum_{i=1}^{M} \varepsilon_{i}=\frac{d+e}{\sqrt{2 a}}\left(y(a)-\frac{1}{q}\right)+1$. Since $y(a)>\frac{1}{q}$ by assumption, we obtain the desired inequality.

Then, using (7.1) and (i), we find

$$
\sqrt{\sigma q} \geqslant \sqrt{\sigma L} \geqslant \frac{d+e}{\sqrt{2 a}}\left(y(a)-\frac{1}{q}\right)+1=\frac{d+e}{\sqrt{2 a}} \delta+1=\delta q v_{M}+1>\delta q v_{M} .
$$

Thus

$$
\sqrt{q}<\frac{\sqrt{\sigma}}{\delta v_{M}}
$$


For both types of classes $(d, d ; m)$ and $\left(d+\frac{1}{2}, d-\frac{1}{2} ; m\right)$, we get

$$
d \leqslant \frac{\sqrt{a}}{\sqrt{2} \delta}(\sqrt{\sigma L}-1) \leqslant \frac{\sqrt{a}}{\sqrt{2} \delta}(\sqrt{\sigma q}-1)<\frac{\sqrt{a}}{\sqrt{2} \delta}\left(\frac{\sigma}{\delta v_{M}}-1\right)
$$

If $v_{M}<1$, the same arguments go through, when replacing $\sigma$ by $\sigma^{\prime}$.

\subsection{The interval $[6,7]$}

We start by stating a more precise version of part (ii) of Theorem 1.3

Theorem 7.2. On the interval $\left[\sigma^{2}, 7 \frac{1}{32}\right], c(a)=\sqrt{\frac{a}{2}}$ except on the seven disjoint intervals $] u_{x}, v_{x}$ [ given in the following table. For each of these intervals, there exist a class $(d, e ; m) \in \mathcal{E}$ and a rational number $x \in] u_{x}, v_{x}[$ with $l(x)=$ $l(m)$ such that $c(z)=\mu(d, e ; m)(z)=\frac{1}{d+e}(A+B z)$ on $\left[u_{x}, x\right]$, and $c(z)=$ $\mu(d, e ; m)(z)=\frac{1}{d+e}\left(A^{\prime}+B^{\prime} z\right)$ on $\left[x, v_{x}\right]$. We list all these informations in the table below as well as the values of $c(x)$ and $\sqrt{\frac{x}{2}}$.

\begin{tabular}{|c|c|c|c|c|c|c|}
\hline$x$ & $(d, e ; m)$ & $(A, B)$ & $\left(A^{\prime}, B^{\prime}\right)$ & $c(x)$ & $c(x) \cong$ & $\sqrt{\frac{x}{2}} \cong$ \\
\hline 6 & $\left(2,2 ; 2,1^{\times 5}\right)$ & $(1,1)$ & $(7,0)$ & $\frac{7}{4}$ & 1.75 & 1.73 \\
\hline $6 \frac{1}{7}$ & $\left(28,28 ; 16^{\times 6}, 3,2^{\times 6}\right)$ & $(6,15)$ & $(92,1)$ & $\frac{687}{392}$ & 1.752551 & 1.752549 \\
\hline $6 \frac{1}{6}$ & $\left(14,14 ; 8^{\times 6}, 2,1^{\times 5}\right)$ & $(6,7)$ & $(43,1)$ & $\frac{295}{168}$ & 1.75595 & 1.75594 \\
\hline $6 \frac{1}{5}$ & $\left(11,10 ; 6^{\times 6}, 1^{\times 5}\right)$ & $(6,5)$ & $(37,0)$ & $\frac{37}{21}$ & 1.762 & 1.761 \\
\hline $6 \frac{1}{3}$ & $\left(7,7 ; 4^{\times 6}, 1^{\times 3}\right)$ & $(6,3)$ & $(25,0)$ & $\frac{25}{14}$ & 1.79 & 1.78 \\
\hline $6 \frac{1}{2}$ & $\left(9,9 ; 5^{\times 6}, 3,2\right)$ & $(0,5)$ & $(26,1)$ & $\frac{65}{36}$ & 1.81 & 1.80 \\
\hline 7 & $\left(4,4 ; 3,2^{\times 6}\right)$ & $(1,2)$ & $(15,0)$ & $\frac{15}{8}$ & 1.88 & 1.87 \\
\hline
\end{tabular}

\begin{tabular}{|c|c|c|c|c|}
\hline$x$ & $u_{x}$ & $u_{x} \cong$ & $v_{x}$ & $v_{x} \cong$ \\
\hline 6 & $\sigma^{2}=3+2 \sqrt{2}$ & 5.83 & $6 \frac{1}{8}=\frac{49}{8}$ & 6.13 \\
\hline $6 \frac{1}{7}$ & $\frac{2}{225}(347+28 \sqrt{151})$ & 6.142842 & $4(173-70 \sqrt{6})$ & 6.142872 \\
\hline $6 \frac{1}{6}$ & $\frac{2}{7}(11+4 \sqrt{7})$ & 6.16657 & $153-14 \sqrt{110}$ & 6.16676 \\
\hline $6 \frac{1}{5}$ & $\frac{3}{100}(107+7 \sqrt{201})$ & 6.19 & $\frac{2738}{441}$ & 6.21 \\
\hline $6 \frac{1}{3}$ & $\frac{1}{9}(31+7 \sqrt{13})$ & 6.2488 & $\frac{625}{98}$ & 6.38 \\
\hline $6 \frac{1}{2}$ & $\frac{162}{25}$ & 6.48 & $55-9 \sqrt{29}$ & 6.53 \\
\hline 7 & $\frac{1}{2}(7+4 \sqrt{3})$ & 6.96 & $7 \frac{1}{32}=\frac{225}{32}$ & 7.03 \\
\hline
\end{tabular}


Lemma 7.3. The classes $(d, e ; m) \in \mathcal{E}$ such that $\mu(d, e ; m)\left(6 \frac{1}{k}\right)>\sqrt{\frac{6 \frac{1}{k}}{2}}$ and $l(m)=l\left(6 \frac{1}{k}\right)$ for some $k=1, \ldots, 8$ are given in the following table.

\begin{tabular}{|c|c|}
\hline$k$ & $(d, e ; m)$ \\
\hline 7 & $\left(28,28 ; 16^{\times 6}, 3,2^{\times 6}\right)$ \\
\hline 7 & $\left(196,196 ; 112^{\times 5}, 111,16^{\times 7}\right)$ \\
\hline 6 & $\left(14,14 ; 8^{\times 6}, 2,1^{\times 5}\right)$ \\
\hline 6 & $\left(84,84 ; 48^{\times 5}, 47,8^{\times 6}\right)$ \\
\hline 5 & $\left(11,10 ; 6^{\times 6}, 1^{\times 5}\right)$ \\
\hline 4 & $\left(28,28 ; 16^{\times 5}, 15,4^{\times 4}\right)$ \\
\hline 3 & $\left(7,7 ; 4^{\times 6}, 1^{\times 3}\right)$ \\
\hline 2 & $\left(9,9 ; 5^{\times 6}, 3,2\right)$ \\
\hline 1 & $\left(4,4 ; 3,2^{\times 6}\right)$ \\
\hline
\end{tabular}

Proof. In the case $k=1$, since $6 \frac{1}{1}=7$, we only have to check which elements of the finite set $\mathcal{E}_{7}$ are obstructive at 7 . It turns out that the only obstructive one is $\left(4,4 ; 3,2^{\times 6}\right)$.

Let us now treat the cases $k=2, \ldots, 8$. Suppose that there is a class of the form $(d, d ; m)$ which is obstructive at some $6 \frac{1}{k}$. Since $l\left(6 \frac{1}{k}\right)=6+k$, by Lemma 4.14 the vector $m$ has to be of one of the five forms

$$
\begin{array}{ccc}
\left(a^{\times 6}, b^{\times k}\right), & \left(a+1, a^{\times 5}, b^{\times k}\right), & \left(a^{\times 5}, a-1, b^{\times k}\right), \\
\left(a^{\times 6}, b+1, b^{\times(k-1)}\right), & \left(a^{\times 6}, b^{\times(k-1)}, b-1\right) .
\end{array}
$$

Define $\varepsilon_{a}$ and $\varepsilon_{b}$ by

$$
a=\frac{\sqrt{2} d}{\sqrt{6 \frac{1}{k}}}+\varepsilon_{a} \quad \text { and } \quad b=\frac{\sqrt{2} d}{k \sqrt{6 \frac{1}{k}}}+\varepsilon_{b} .
$$

If $m=\left(a^{\times 6}, b^{\times k}\right)$, then $|a-k b|=\left|\varepsilon_{a}-k \varepsilon_{b}\right| \leqslant\left|\varepsilon_{a}\right|+k\left|\varepsilon_{b}\right|$. Since by Lemma 4.8 (iii), $\sum \varepsilon_{i}^{2}<1$, we find $\left|\varepsilon_{a}\right|+k\left|\varepsilon_{b}\right|<\sqrt{k+1}$, and thus $|a-k b| \leqslant$ $\lceil\sqrt{k+1}-1\rceil$. Hence

$$
s:=a-k b \in \begin{cases}\{0, \pm 1\} & \text { if } k \in\{2,3\} \\ \{0, \pm 1, \pm 2\} & \text { if } k \in\{4, \ldots, 8\}\end{cases}
$$


The Diophantine equations of Proposition 3.8(i) then become

$$
\begin{aligned}
4 d & =6 a+k b+1, \\
2 d^{2} & =6 a^{2}+k b^{2}-1 .
\end{aligned}
$$

Thus $(6 a+k b+1)^{2}=8\left(6 a^{2}+k b^{2}-1\right)$. Replacing $a$ by $k b+s$, we can solve this equation in $b$ for the values of $k$ and $s$ given above. We find three solutions to the equation with $b \geqslant 1$, namely when $(k, s, b)$ is equal to $(3,0,3),(3,1,1)$ or $(3,-1,5)$. This leads to the vectors $\left(16,16 ; 9^{\times 6}, 3^{\times 3}\right),\left(7,7 ; 4^{\times 6}, 1^{\times 3}\right)$ and $\left(25,25 ; 14^{\times 6}, 5^{\times 3}\right)$, respectively. Since only $\left(7,7 ; 4^{\times 6}, 1^{\times 3}\right)$ reduces to $(0 ;-1,0$, $\ldots, 0)$ by Cremona moves, this is the only class of the form $\left(d, d ; a^{\times 6}, b^{\times k}\right)$ potentially obstructive at some $6 \frac{1}{k}$, and it indeed is obstructive at $6 \frac{1}{3}$.

In the case where $m=\left(a+1, a^{\times 5}, b^{\times k}\right), \sigma=k\left|\varepsilon_{b}\right|^{2} \leqslant \frac{1}{6}$. Thus, $|a-k b| \leqslant$ $\left|\varepsilon_{a}\right|+k\left|\varepsilon_{b}\right| \leqslant 1+\sqrt{\frac{k}{6}}$, and thus

$$
s:=a-k b \in \begin{cases}\{0, \pm 1\} & \text { if } k \in\{2, \ldots, 5\} \\ \{0, \pm 1, \pm 2\} & \text { if } k \in\{6, \ldots, 8\}\end{cases}
$$

From the Diophantine equations we obtain

$$
(6 a+k b+2)^{2}=8\left(6 a^{2}+2 a+k b^{2}\right)
$$

Replacing $a$ by $k b+s$, we obtain no solutions with $b \geqslant 1$ for the accepted values of $k$ and $s$.

As in the previous case, when $m=\left(a^{\times 5}, a-1, b^{\times k}\right)$, we have

$$
s:=a-k b \in \begin{cases}\{0, \pm 1\} & \text { if } k \in\{2, \ldots, 5\} \\ \{0, \pm 1, \pm 2\} & \text { if } k \in\{6, \ldots, 8\}\end{cases}
$$

The Diophantine equations become $(6 a+k b)^{2}=8\left(6 a^{2}-2 a+k b^{2}\right)$, which yields four solutions with $b \geqslant 1$, namely the tuples $(k, s, b)$ equal to $(2,1,1)$, $(4,0,4),(6,0,8)$ and $(7,0,16)$ which give the vectors $\left(5,5 ; 3^{\times 5}, 2,1^{\times 2}\right),(28,28$; $\left.16^{\times 5}, 15,4^{\times 4}\right),\left(84,84 ; 48^{\times 5}, 47,8^{\times 6}\right)$ and $\left(196,196 ; 112^{\times 5}, 111,16^{\times 7}\right)$, respectively. These vectors all reduce to $(0 ;-1,0, \ldots, 0)$ by Cremona moves, but the first one is not obstructive at $6 \frac{1}{2}$. So we add only the three last vectors to our table.

For the case $m=\left(a^{\times 6}, b+1, b^{\times(k-1)}\right)$ notice that if $\varepsilon \in \mathbb{R}$ and $k \in \mathbb{N}$ are such that $(k-1) \varepsilon^{2}+(\varepsilon+1)^{2} \leqslant 1$, then $\varepsilon \in\left[-\frac{2}{k}, 0\right]$. Thus, $|(k-1) \varepsilon+(\varepsilon+1)|$ 
$=|k \varepsilon+1| \leqslant 1$. Since $\sigma \geqslant \frac{k-1}{k}$, we get

$$
\begin{aligned}
|a-k b-1| & =|a-(b+1)-(k-1) b|=\left|\varepsilon_{a}-\left(\varepsilon_{b}+1\right)-(k-1) \varepsilon_{b}\right| \\
& \leqslant\left|\varepsilon_{a}\right|+\left|(k-1) \varepsilon_{b}+\varepsilon_{b}+1\right| \leqslant 1+1=2 .
\end{aligned}
$$

Thus

$$
s:=a-k b-1 \in\{0, \pm 1, \pm 2\} .
$$

The Diophantine equations become $(6 a+k b+2)^{2}=8\left(6 a^{2}+k b^{2}+2 b\right)$, which when we replace $a$ by $k b+s+1$ gives the three tuples of solutions $(k, s, b)$ equal to $(2,0,2),(6,1,6)$ and $(7,1,2)$, which yields the vectors $\left(9,9 ; 5^{\times 6}, 3,2\right)$, $\left(14,14 ; 8^{\times 6}, 2,1^{\times 5}\right)$ and, again, $\left(28,28 ; 16^{\times 6}, 3,2^{\times 6}\right)$, respectively. All three vectors reduce to $(0 ;-1,0, \ldots, 0)$ by Cremona moves, and they are obstructive at $6 \frac{1}{k}$ for $k=2,6,7$ respectively.

For the case $m=\left(a^{\times 6}, b^{\times(k-1)}, b-1\right)$ we find similarly as in the previous case that

$$
s:=a-k b+1 \in\{0, \pm 1, \pm 2\} .
$$

The Diophantine equations become $(6 a+k b)^{2}=8\left(6 a^{2}+k b^{2}-2 b\right)$, which when we replace $a$ by $k b+s-1$ gives as only solution with $b \geqslant 2$ the tuple $(k, s, b)=(2,0,3)$. This gives again the vector $\left(9,9 ; 5^{\times 6}, 3,2\right)$.

The last case we have to treat is the case of an obstructive class of the form $\left(d+\frac{1}{2}, d-\frac{1}{2} ; m\right)$. By Corollary 4.15 the only possibility for $m$ is to be of the form $\left(a^{\times 6}, b^{\times k}\right)$. We saw earlier that in this case we have

$$
s:=a-k b \in \begin{cases}\{0, \pm 1\} & \text { if } k \in\{2,3\} \\ \{0, \pm 1, \pm 2\} & \text { if } k \in\{4, \ldots, 8\}\end{cases}
$$

Now the Diophantine equations are

$$
\begin{aligned}
4 d & =6 a+k b+1 \\
2 d^{2} & =6 a^{2}+k b^{2}-\frac{1}{2} .
\end{aligned}
$$

This leads to the equation $\frac{1}{8}(6 a+k b+3)^{2}-\frac{1}{2}(6 a+k b+3)+1=6 a^{2}+k b^{2}$. When replacing $a$ by $k b+s$, we obtain as only solution with $b \geqslant 1$ the tuple $(5,1,1)$ which gives the vector $\left(11,10 ; 6^{\times 6}, 1^{\times 5}\right)$. This vector reduces to $(0$; $-1,0, \ldots, 0)$ by Cremona moves and is obstructive at $6 \frac{1}{5}$.

Lemma 7.4. The classes given in Lemma 7.3 are the only obstructive classes on the interval $\left[6 \frac{1}{8}, 7\right]$. 
Proof. We claim that it suffices to prove that for all $a \in\left[6 \frac{1}{8}, 7\right]$, there is no other class $(d, e ; m) \in \mathcal{E}$ with $l(m)=l(a)$ that is obstructive at $a$. Indeed, suppose that $\mu(d, e ; m)(a)>\sqrt{\frac{a}{2}}$ for some $a \in\left[6 \frac{1}{8}, 7\right]$, and let $I$ be the maximal nonempty interval containing $a$ on which $\mu(d, e ; m)(z)>\sqrt{\frac{z}{2}}$. Then, by Lemma 4.13 there exists a unique $a_{0} \in I$ such that $l\left(a_{0}\right)=l(m)$ and $l\left(a_{0}\right) \leqslant l(a)$ for all $a \in I$. Since for $M \leqslant 6, \mathcal{E}_{M}$ is finite, explicit calculations show that none of these classes is obstructive for $a \geqslant 6 \frac{1}{8}$. Thus $l(m)>6$, and $a_{0}>6$. This implies that $a_{0} \geqslant 6 \frac{1}{8}$. Indeed, $a_{0}<6 \frac{1}{8}$ would contradict the fact that $l\left(a_{0}\right) \leqslant l(a)$ for all $a \in I$ since $l(a)>l\left(6 \frac{1}{8}\right)$ for all $\left.a \in\right] 6,6 \frac{1}{8}[$. A similar argument also shows that $a_{0} \leqslant 7$. Thus, $a_{0} \in\left[6 \frac{1}{8}, 7\right]$ and this proves the claim.

We will thus prove that for each $a=6 \frac{p}{q} \in\left[6 \frac{1}{8}, 7\right]$ there is no class $(d, e$; $m) \in \mathcal{E}$ with $l(m)=l(a)$ obstructive at $a$, and different from those given in Lemma 7.3. By Lemma 7.3, we only have to prove for $\frac{p}{q} \neq \frac{1}{k}$ with $k=1, \ldots, 8$. We will separate the proot in three cases: $3 \leqslant q \leqslant 8,9 \leqslant q \leqslant 39, q \geqslant 40$.

Case 1: $3 \leqslant q \leqslant 8$ : In this case, $2 \leqslant p \leqslant q$. Notice that for all these values of $p$ and $q, y\left(6 \frac{p}{q}\right)>\frac{1}{q}$. We can thus apply Lemma 7.1 (iv). We get that if $(d, e ; m) \in \mathcal{E}$ is obstructive at $6 \frac{p}{q}$, then

$$
d \leqslant \frac{\sqrt{6 \frac{p}{q}}}{\sqrt{2}\left(y\left(6 \frac{p}{q}\right)-\frac{1}{q}\right)}(\sqrt{q}-1)
$$

since $\sigma<1$ for an obstructive class. We now use the computer program SolLess $[\mathrm{a}, \mathrm{D}]$ given in the Appendix which computes for a rational number $a$ and a natural number $D$ all obstructive classes $(d, e ; m)$ at $a$ with $l(m)=l(a)$ and $d \leqslant D$. The code shows that there are no such classes for $3 \leqslant q \leqslant 8$. have

Case 2: $9 \leqslant q \leqslant 39$ : Since $y\left(6 \frac{1}{8}\right)=\frac{1}{8}$ and $y$ is increasing for $a>2$, we

$$
y(a)-\frac{1}{q} \geqslant \frac{1}{8}-\frac{1}{9}>0
$$

for all $a \in\left[6 \frac{1}{8}, 7\right]$. We can thus again apply Lemma 7.1 (iv) and obtain again (7.2), but this time for $1 \leqslant p \leqslant q$. Again, the code SolLess[a, D] shows that for $9 \leqslant q \leqslant 39$ there are no obstructive classes $(d, e ; m)$ at $a=6 \frac{p}{q}$ with $l(m)=$ $l(a)$.

Case 3: $q \geqslant 40$ : For all $a=6 \frac{p}{q} \in\left[6 \frac{1}{8}, 7\right]$, we have $\delta:=y(a)-\frac{1}{q} \geqslant \frac{1}{8}-$ $\frac{1}{40}=\frac{1}{10}$. Suppose that $(d, e ; m) \in \mathcal{E}$ is obstructive at some $a=6 \frac{p}{q}$ with $q \geqslant 40$. We distinguish two cases: (i) $m_{1}=m_{6}$, (ii) $m_{1} \neq m_{6}$. 
(i) Notice that by Lemma 7.1 (iii),

$$
\begin{array}{ll}
\text { if } v_{M} \in\left[\frac{1}{3}, \frac{1}{2}\right], & \text { then } \frac{\sigma^{\prime}}{v_{M}} \leqslant \frac{1 / 2}{1 / 3}=\frac{3}{2}, \\
\text { if } v_{M} \in\left[\frac{1}{2}, \frac{2}{3}\right], & \text { then } \frac{\sigma^{\prime}}{v_{M}} \leqslant \frac{7 / 8}{1 / 2}=\frac{7}{4}, \\
\text { if } v_{M} \geqslant \frac{2}{3}, & \text { then } \frac{\sigma}{v_{M}} \leqslant \frac{3}{2} .
\end{array}
$$

By Lemma 7.1 (iv) we get that if $\left.a=6 \frac{p}{q} \in\right] 6 \frac{1}{k+1}, 6 \frac{1}{k}[$ for some $k=1, \ldots, 7$ and $q \geqslant 40$, then for all obstructive classes $(d, e ; m)$ at $a$ with $m_{1}=m_{6}$

$$
d \leqslant \frac{\sqrt{6 \frac{1}{k}}}{\sqrt{2}\left(y\left(6 \frac{1}{k+1}\right)-\frac{1}{40}\right)}\left(\frac{1}{y\left(6 \frac{1}{k+1}\right)-\frac{1}{40}} \frac{7}{4}-1\right)
$$

Here we used the computer program InterSolLess1[k, D] given in the Appendix which gives for $k \in\{1, \ldots, 7\}$ and a natural number $D$ a finite list of classes $(d, e ; m)$ with $m_{1}=m_{6}$ and $d \leqslant D$ which can potentially be obstructive at some $\left.a=6 \frac{p}{q} \in\right] 6 \frac{1}{k+1}, 6 \frac{1}{k}[$ with $q \geqslant 40$. Applied to our case, the code gives only one class that reduces to $(0 ;-1,0, \ldots, 0)$ by Cremona moves, namely $(d, e ; m)=\left(99,99 ; 56^{\times 6}, 14^{\times 4}, 1^{\times 3}\right)$. By Lemma 4.14 the $a$ in question can be $[6 ; 3,1,3]=6 \frac{4}{15}$ or $[6,3,1,1,2]=6 \frac{5}{18}$, and the class turns out to give no obstruction at these two points.

(ii) Since $m_{1} \neq m_{6}$, we know by Lemma 4.14 that $\sigma \leqslant \frac{1}{6}$. This implies that $v_{M} \geqslant 1-\frac{1}{2 \sqrt{3}}$ because the last two weights of $w\left(\frac{p}{q}\right)$ are always $\frac{1}{q}$. Then by Lemma 7.1 (iv) we get that if $\left.a=6 \frac{p}{q} \in\right] 6 \frac{1}{k+1}, 6 \frac{1}{k}$ [ for some $k=1, \ldots, 7$ and $q \geqslant 40$, then for all obstructive classes $(d, e ; m)$ at $a$ with $m_{1} \neq m_{6}$ we have

$$
d \leqslant \frac{\sqrt{6 \frac{1}{k}}}{\sqrt{2}\left(y\left(6 \frac{1}{k+1}\right)-\frac{1}{40}\right)}\left(\frac{1}{y\left(6 \frac{1}{k+1}\right)-\frac{1}{40}} \frac{\frac{1}{6}}{\left(1-\frac{1}{2 \sqrt{3}}\right)}-1\right)
$$

Here we used the computer program InterSolLess $2[k, D]$ which gives for $k \in\{1, \ldots, 7\}$ and a natural number $D$ a finite list of classes $(d, e ; m)$ with $m_{1} \neq m_{6}$ and $d \leqslant D$ which can potentially be obstructive at some $a=6 \frac{p}{q} \in$ ] $6 \frac{1}{k+1}, 6 \frac{1}{k}[$ with $q \geqslant 40$. Applied to our case, the code gives no class that reduces to $(0 ;-1,0, \ldots, 0)$ by Cremona moves.

Remark 7.5. The three programs SolLess[a,D], InterSolLess $1[k, D]$ and InterSolLess $2[\mathrm{k}, \mathrm{D}]$ give, for a natural number $D$, solutions $(d, e ; m)$ with $d \leqslant$ 
$D$. But, in the case of classes of the form $\left(d+\frac{1}{2}, d-\frac{1}{2} ; m\right)$, we give estimates for $d$ in Lemma 7.4 Thus for these classes, we have to add $\frac{1}{2}$ to our estimates when using the programs.

Proof of Theorem 7.2. We have already proven in Theorem 6.21 that the class $\left(2,2 ; 2,1^{\times 5}\right)$ gives the constraint $c(a)=\mu\left(2,2 ; 2,1^{\times 5}\right)(a)=\frac{a+1}{4}$ on $\left[\sigma^{2}, 6\right]$. We postpone the proof that $c(a)=\mu\left(4,4 ; 3,2^{\times 6}\right)(a)=\frac{15}{8}$ on $\left[7,7 \frac{1}{32}\right]$ to Corollary 7.8

Since by Lemma 7.4 , the only obstructive classes on the interval $\left[6 \frac{1}{8}, 7\right]$ are those of Lemma 7.3. shows that none of them is obstructive at $6 \frac{1}{8}$. Hence, $c(a)=\frac{7}{4}$ for all $a \in$ $\left[6,6 \frac{1}{8}\right]$ since $c$ is nondecreasing.

In order to determine $c$ on the interval $\left[6 \frac{1}{8}, 7\right]$, Lemma 7.4 shows that we only have to work out the constraints given by the classes of Lemma 7.3 . Notice that for $a \in] 6 \frac{1}{k+1}, 6 \frac{1}{k}[$, the first terms of the weight expansion of $a$ are $w(a)=\left(1^{\times 6},(a-6)^{\times k}, 1-k(a-6), \ldots\right)$. We can thus easily compute the constraints of all the classes. In the next table, we write the constraints given by the classes of Lemma 7.3 that do not appear in Theorem 7.2 and we then simply verify that they indeed do not give new obstructions.

\begin{tabular}{|c|c|c|c|c|}
\hline$x$ & $(d, e ; m)$ & $(A, B)$ & $\left(A^{\prime}, B^{\prime}\right)$ & $\mu(x)$ \\
\hline $6 \frac{1}{7}$ & $\left(196,196 ; 112^{\times 5}, 111,16^{\times 7}\right)$ & $(-1,112)$ & $(687,0)$ & $\frac{687}{392}$ \\
\hline $6 \frac{1}{6}$ & $\left(84,84 ; 48^{\times 5}, 47,8^{\times 6}\right)$ & $(-1,48)$ & $(295,0)$ & $\frac{295}{168}$ \\
\hline $6 \frac{1}{4}$ & $\left(28,28 ; 16^{\times 5}, 15,4^{\times 4}\right)$ & $(-1,16)$ & $(99,0)$ & $\frac{99}{56}$ \\
\hline
\end{tabular}

\begin{tabular}{|c|c|c|c|c|}
\hline$x$ & $u_{x}$ & $u_{x} \cong$ & $v_{x}$ & $v_{x} \cong$ \\
\hline $6 \frac{1}{7}$ & $\frac{1}{112}(344+7 \sqrt{2415})$ & 6.142844 & $\frac{471969}{76832}$ & 6.142870 \\
\hline $6 \frac{1}{6}$ & $\frac{1}{48}(148+7 \sqrt{447})$ & 6.16660 & $\frac{87025}{14112}$ & 6.16674 \\
\hline $6 \frac{1}{4}$ & $\frac{1}{16}(50+7 \sqrt{51})$ & 6.2494 & $\frac{9801}{1568}$ & 6.25 \\
\hline
\end{tabular}

The proof of Theorem 7.2 (up to Corollary 7.8) is complete.

\subsection{The interval $[7,8]$}

Lemma 7.6. Assume that there exists a class $(d, e ; m) \in \mathcal{E}$ such that $\mu(d, e$; $m)(a)>\sqrt{\frac{a}{2}}$ for some $a \in\left[7 \frac{1}{32}, 8\right]$ with $l(a)=l(m)$. Then $m_{1}=\cdots=m_{7}$ and $d \leqslant 13$. 
Proof. Notice first that

$$
y(a) \geqslant y\left(7 \frac{1}{32}\right)=\frac{17}{32}>\frac{1}{q}
$$

for all $q \geqslant 2$. We distinguish two cases: $q \geqslant 12$ and $q \leqslant 11$.

If $q \geqslant 12$, then $\delta=y(a)-\frac{1}{q} \geqslant \frac{17}{32}-\frac{1}{12}=\frac{43}{96}$. Assume by contradiction that $m_{1} \neq m_{7}$. Then by Lemma $4.14 \sigma \leqslant \frac{1}{7}$ and so $v_{M} \geqslant \frac{1}{2}$. Thus,

$$
\frac{\sigma}{v_{M} \delta} \leqslant \frac{192}{301}<1
$$

But this contradicts Lemma 7.1(iv).

To prove that $d \leqslant 13$, notice first that by Lemma 7.1(iii),

$$
\begin{array}{ll}
\text { if } v_{M} \in\left[\frac{1}{3}, \frac{1}{2}\right], & \text { then } \frac{\sigma^{\prime}}{v_{M}} \leqslant \frac{1 / 2}{1 / 3}=\frac{3}{2}, \\
\text { if } v_{M} \in\left[\frac{1}{2}, \frac{2}{3}\right], & \text { then } \frac{\sigma^{\prime}}{v_{M}} \leqslant \frac{7 / 8}{1 / 2}=\frac{7}{4}, \\
\text { if } v_{M} \geqslant \frac{2}{3}, & \text { then } \frac{\sigma}{v_{M}} \leqslant \frac{3}{2} .
\end{array}
$$

Then, since $\sqrt{a} \leqslant 2 \sqrt{2}$, we get by Lemma 7.1 (iv) that

$$
d \leqslant \frac{2 \sqrt{2}}{43 / 96 \sqrt{2}}\left(\frac{1}{43 / 96} \frac{7}{4}-1\right)+\frac{1}{2}<14
$$

Thus $d \leqslant 13$.

Let now $q \leqslant 11$. Notice that $a \leqslant 7 \frac{q-1}{q}$ and

$$
\delta=y(a)-\frac{1}{q} \geqslant y\left(7 \frac{1}{q}\right)-\frac{1}{q}
$$

By Lemma 7.1 (iv), we have

$$
d \leqslant \frac{\sqrt{a}}{\sqrt{2} \delta}(\sqrt{\sigma q}-1)+\frac{1}{2} \leqslant \frac{\sqrt{7 \frac{q-1}{q}}}{\sqrt{2}\left(y\left(7 \frac{1}{q}\right)-\frac{1}{q}\right)}(\sqrt{q}-1)+\frac{1}{2}
$$

Since the RHS is strictly smaller than 11 for all $2 \leqslant q \leqslant 11$, we see that $d \leqslant 10$. 
Assume now by contradiction that $m_{1} \neq m_{7}$. Then $\sigma \leqslant \frac{1}{7}$. If $2 \leqslant q \leqslant 7$, then $\sqrt{\sigma q}-1 \leqslant 0$ which contradicts Lemma 7.1 (iv). If $8 \leqslant q \leqslant 11$, then

$$
v_{M}=\frac{d+e}{q \sqrt{2 a}} \leqslant \frac{\sqrt{2} d}{q \sqrt{a}} \leqslant \frac{\sqrt{2} 10}{8 \sqrt{7}},
$$

and so, by Lemma 4.14 .

$$
\langle\varepsilon, \varepsilon\rangle \geqslant \frac{6}{7}+2\left(1-v_{M}\right)^{2}>1
$$

which contradicts Lemma 4.8 (iii).

Proposition 7.7. $c(a)=\sqrt{\frac{a}{2}}$ for all $a \in\left[7 \frac{1}{32}, 8\right]$.

Proof. Suppose by contradiction that there exists $a \geqslant 7 \frac{1}{32}$ and $(d, e ; m) \in \mathcal{E}$ with $\mu(d, e ; m)(a)>\sqrt{\frac{a}{2}}$. Let $I$ be the maximal open interval containing $a$ on which $(d, e ; m)$ is obstructive. Then, by Lemma 4.13 there exists $a_{0} \in I$ with $l\left(a_{0}\right)=l(m)$ and $l(a) \geqslant l\left(a_{0}\right)$ for all $a \in I$.

Using the finite list of $\mathcal{E}_{7}$ in Lemma 4.3 we check by hand that no class in $\mathcal{E}_{7}$ is obstructive for $a \geqslant 7 \frac{1}{32}$. Thus $l(m)>7$ and so $a_{0}>7$. But then $a_{0} \geqslant 7 \frac{1}{32}$. Indeed, assume by contradiction that $a_{0}<7 \frac{1}{32}$. Then since $a_{0}, a \in I, 7 \frac{1}{32}$ will also belong to $I$. But, for all $z \in] 7,7 \frac{1}{32}\left[, l(z)>l\left(7 \frac{1}{32}\right)\right.$, and this contradicts the fact that $l(a) \geqslant l\left(a_{0}\right)$ for all $a \in I$.

Now by Lemma 7.6 , we find that $d \leqslant 13$ and $m_{1}=\cdots=m_{7}$. Since there are only finitely many classes satisfying these conditions, we can compute them explicitly. We find that there is only one class satisfying the conditions, namely $\left(8,7 ; 4^{\times 7}, 1\right)$, but this class is not obstructive for $a \geqslant 7 \frac{1}{32}$.

Corollary 7.8. $c(a)=\frac{15}{8}$ for all $a \in\left[7,7 \frac{1}{32}\right]$.

Proof. Since the class $(d, e ; m)=\left(4,4 ; 3,2^{\times 6}\right)$ gives the constraint

$$
\mu(d, e ; m)(a)=\frac{15}{8}=\sqrt{\frac{7 \frac{1}{32}}{2}}
$$

for all $a \geqslant 7$, we see that $c(a)=\frac{15}{8}$ on $\left[7,7 \frac{1}{32}\right]$ because $c$ is nondecreasing by Lemma 4.2 


\section{Appendix A. Computer programs}

\section{A.1. Computing $c$ at a point $a \in\left[6 \frac{1}{8}, 7\right]$}

We used the computer in Lemma 7.4 to compute $c$ at points $\frac{p}{q} \in\left[6 \frac{1}{8}, 7\right]$ with $q \leqslant 39$. In this section, we explain the code SolLess $[\mathrm{a}, \mathrm{D}]$ which computes for a rational number $a$ and a natural number $D$ all classes $(d, e ; m)$ obstructive at $a$, with $l(m)=l(a)$ and $d \leqslant D$. We have just adapted the program SolLess[a,D] given in the Appendix of [MS] to our case. The modules W[a], $\mathrm{P}[\mathrm{k}]$, Difference[M] are exactly the same as in [MS].

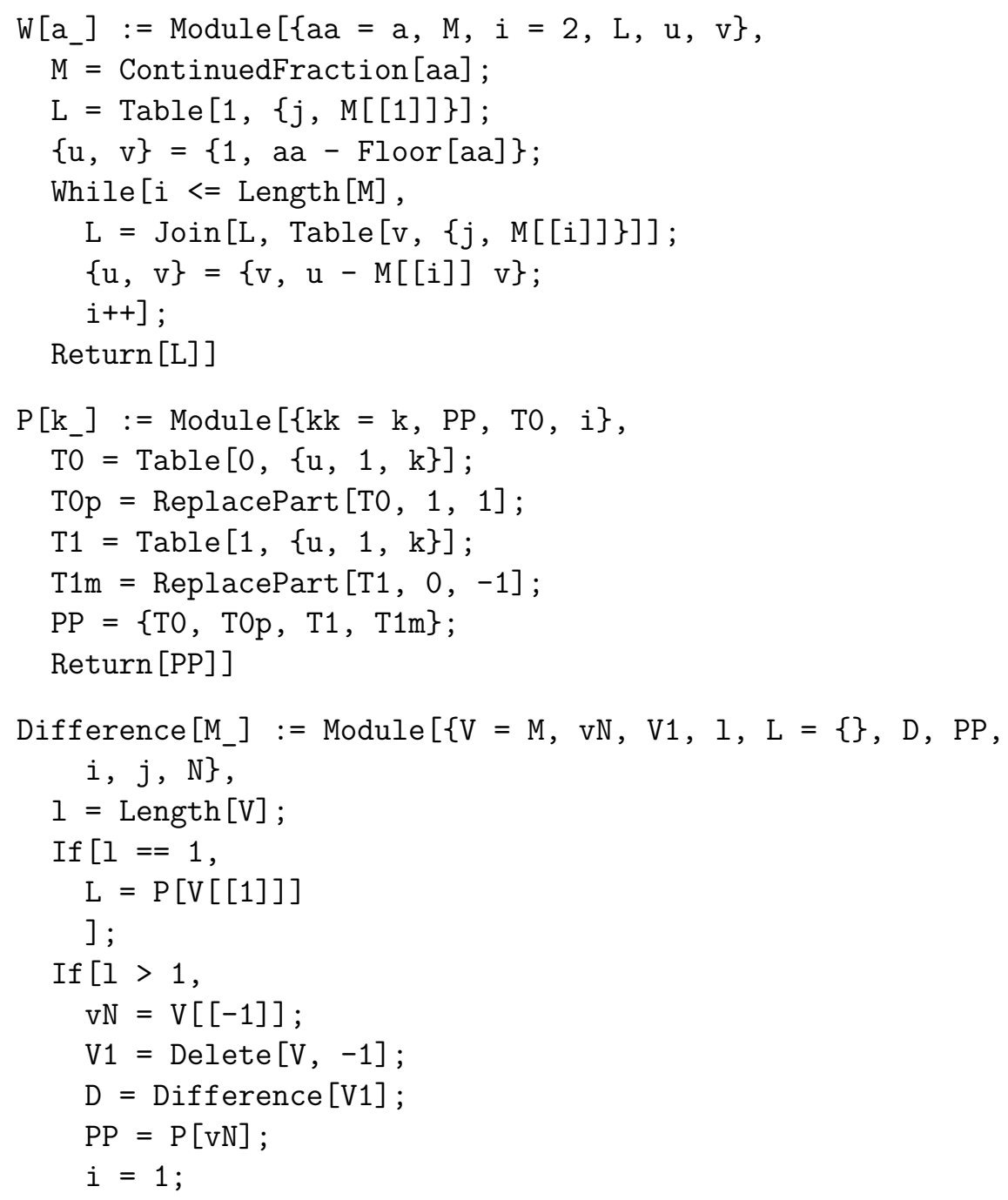




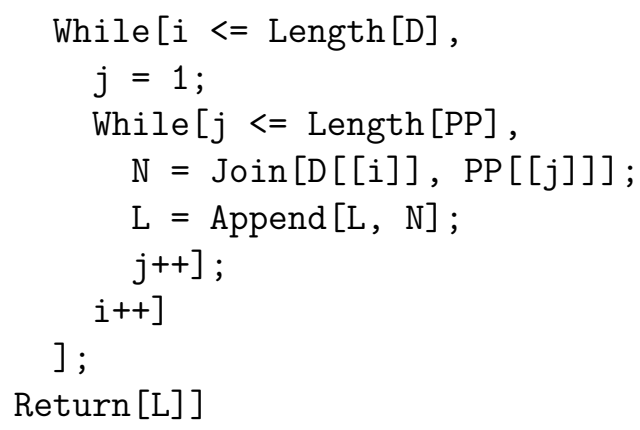

The following module Sol0[a,d] gives for a rational number $a$ all vectors of the form $(d, d ; m)$ with $l(m)=l(a)$ which satisfy the Diophantine equations of Proposition $3.8(\mathrm{i})$ and such that $\mu(d, d ; m)(a)>\sqrt{\frac{a}{2}}$. The code Sol1[a, d] does the same thing for a class of the form $(d, d-1 ; m)$. Note that both modules do not verify whether the vectors reduce to $(0 ;-1)$ by repeated Cremona moves. We have just adapted the code Sol[a,d] of [MS], using that in our case, the volume constraint is $\sqrt{\frac{a}{2}}$ instead of $\sqrt{a}$ and that for a class of the form $(d, d ; m)$ the Diophantine equations become

$$
\sum m_{i}=4 d-1, \quad \sum m_{i}^{2}=2 d^{2}+1,
$$

and for a class of the form $(d, d-1 ; m)$, they become

$$
\sum m_{i}=4 d-3, \quad \sum m_{i}^{2}=2 d^{2}-2 d+1 .
$$

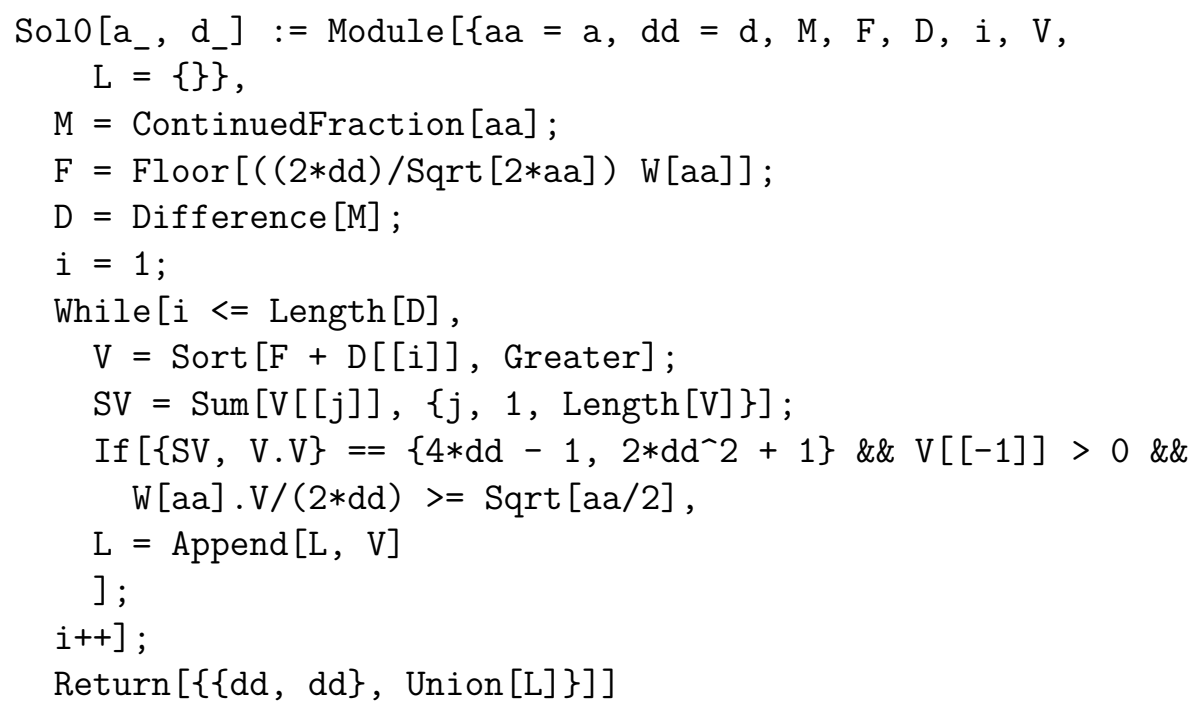




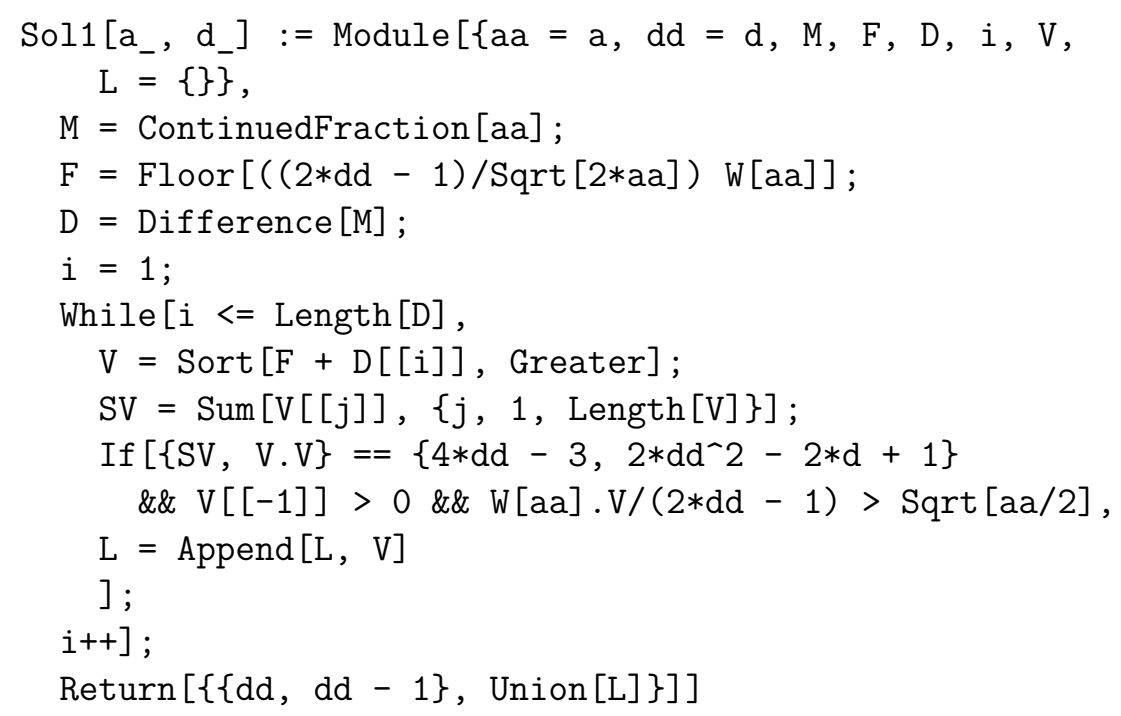

Finally, we collect in the code SolLess $[\mathrm{a}, \mathrm{D}]$ the vectors $(d, e ; m)$ with $l(m)=l(a)$ that are obstructive at $a$ and such that $d \leqslant D$.

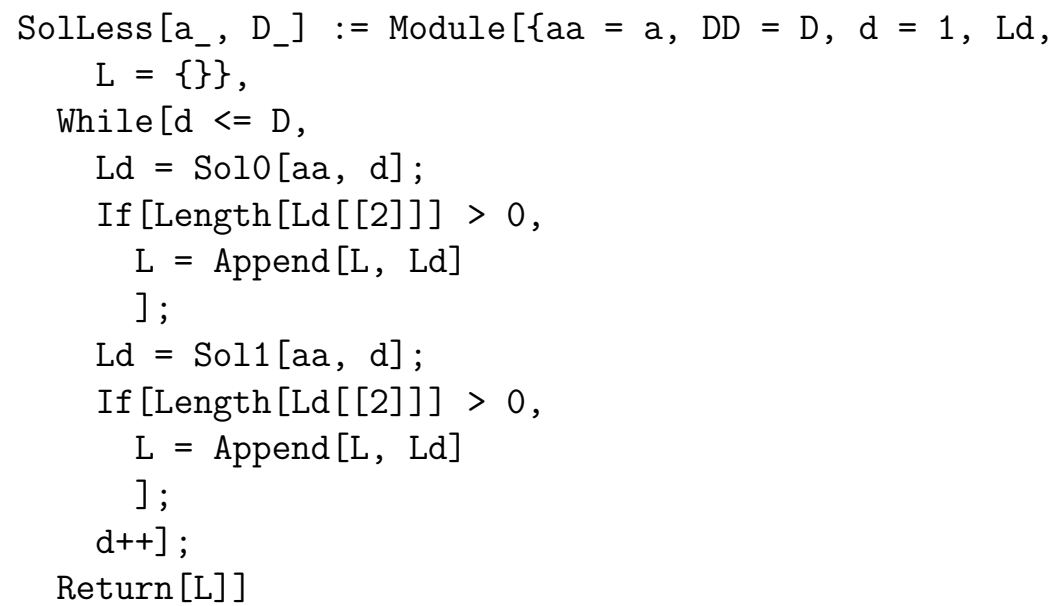

\section{A.2. Computing $c$ on an interval $] 6 \frac{1}{k+1}, 6 \frac{1}{k}[$} with $k \in\{1, \ldots, 7\}$

In Lemma 7.4 we used the codes InterSolLess1[k, D] and InterSolLess2[k, D] which give for $k \in\{1, \ldots, 7\}$ and a natural number $D$, a finite list of vectors $(d, e ; m)$ with $d \leqslant D$ which can potentially be obstructive at some $a \in$ ] $6 \frac{1}{k+1}, 6 \frac{1}{k}[$. By Lemma 4.14 if a class $(d, e ; m) \in \mathcal{E}$ is obstructive at some point $a \in\left[6 \frac{1}{8}, 7\right]$, then we have three possibilities: 
(i) $m_{1}=\cdots=m_{6}$,

(ii) $m_{1}-1=m_{2}=\cdots=m_{6}$,

(iii) $m_{1}=\cdots=m_{5}=m_{6}+1$.

The code InterSolLess1[k, D] treats the case (i) while the cases (ii) \& (iii) are covered by InterSolLess $2[\mathrm{k}, \mathrm{D}]$. We used the programs Solutions[a, b] and sum $[\mathrm{L}]$ exactly as they were in [MS. Solutions $[\mathrm{a}, \mathrm{b}]$ gives for $a, b \in \mathbb{N}$ all vectors $m$ which are solution of the equations

$$
\sum m_{i}=a, \quad \sum m_{i}^{2}=b,
$$

and sum[L] computes the sum of the entries of a vector $L$.

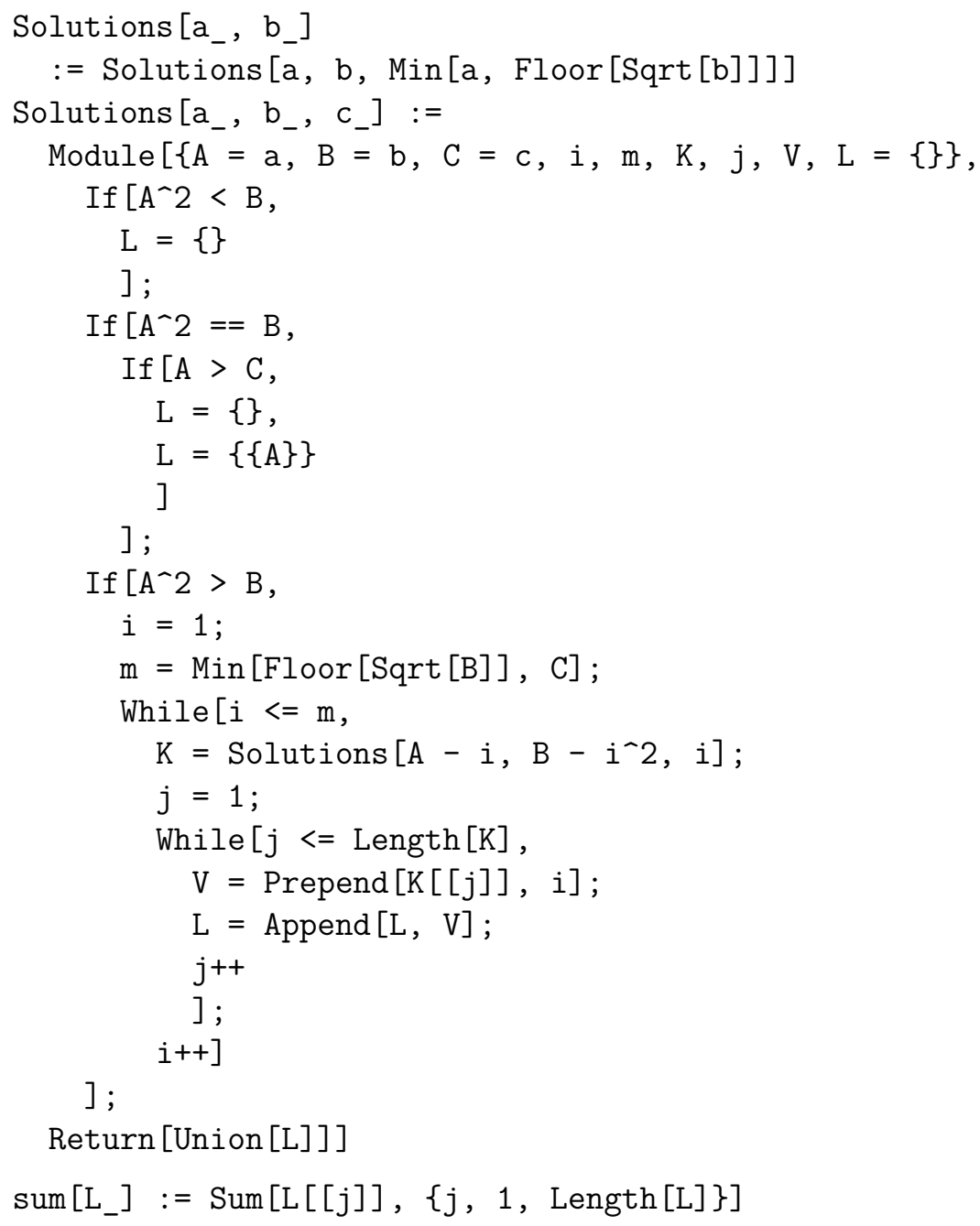


A.2.1. Finding obstructive classes $(d, e ; m)$ with $m_{1}=\cdots=m_{6}$. We have adapted the modules $\mathrm{P}[\mathrm{k}]$, Prelist $[\mathrm{k}, \mathrm{d}]$ from [MS] to the fact that the first six entries of $m$ have to be equal instead of the first seven entries as it was the case in [MS. The module Prelist $[k, d]$ becomes Prelist $[k, d, c]$ where $c=0$ in the case of a class of the form $(d, d ; m)$ and $c=1$ when the class is of the form $(d, d-1 ; m)$. As before, we have adapted the code to take into account that we have another volume constraint and other Diophantine equations. Note that [MS] used their Lemma 2.1.7 and Lemma 2.1.8 which are also true in our case as stated in Lemma 4.14 and Lemma 4.16.

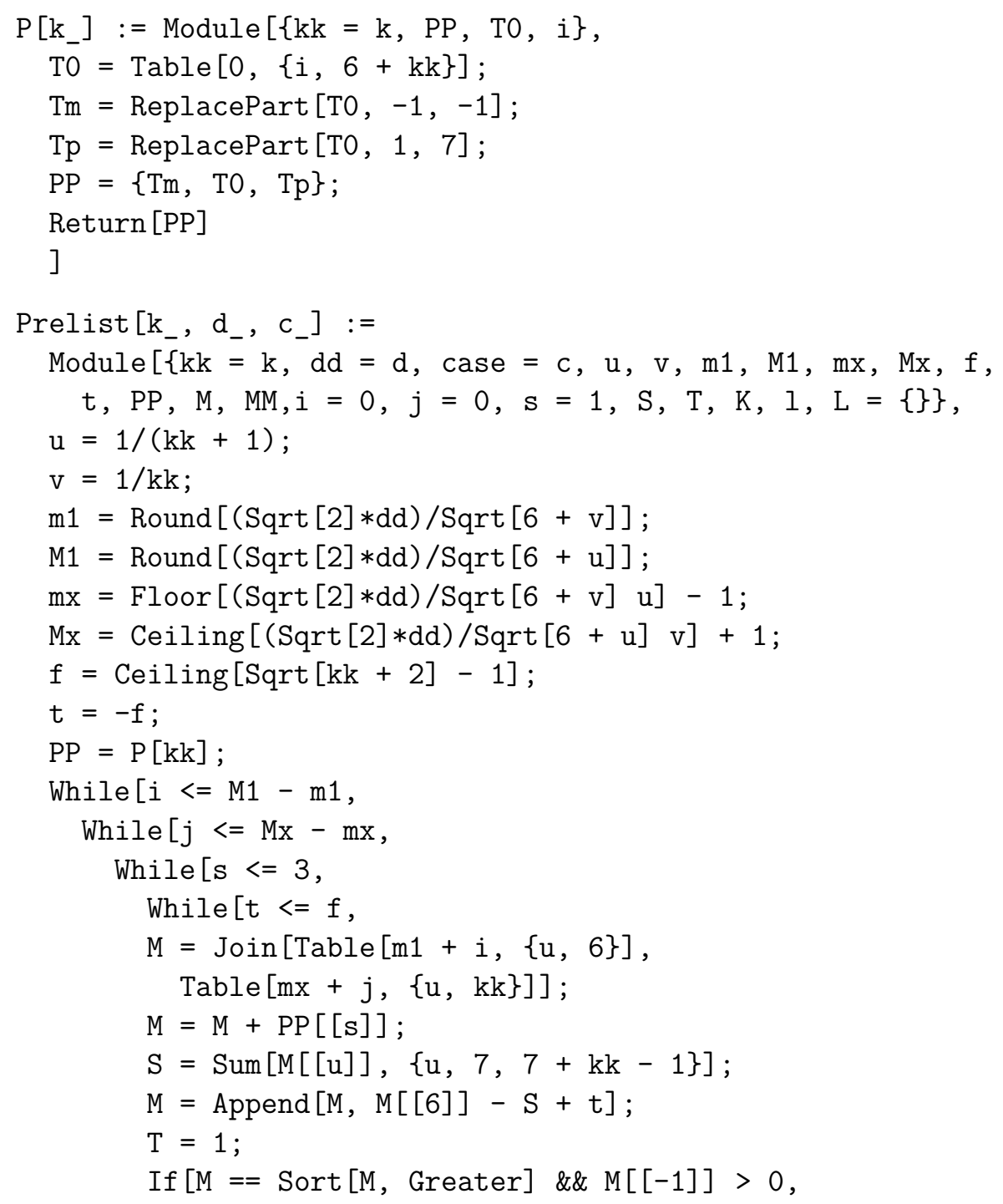




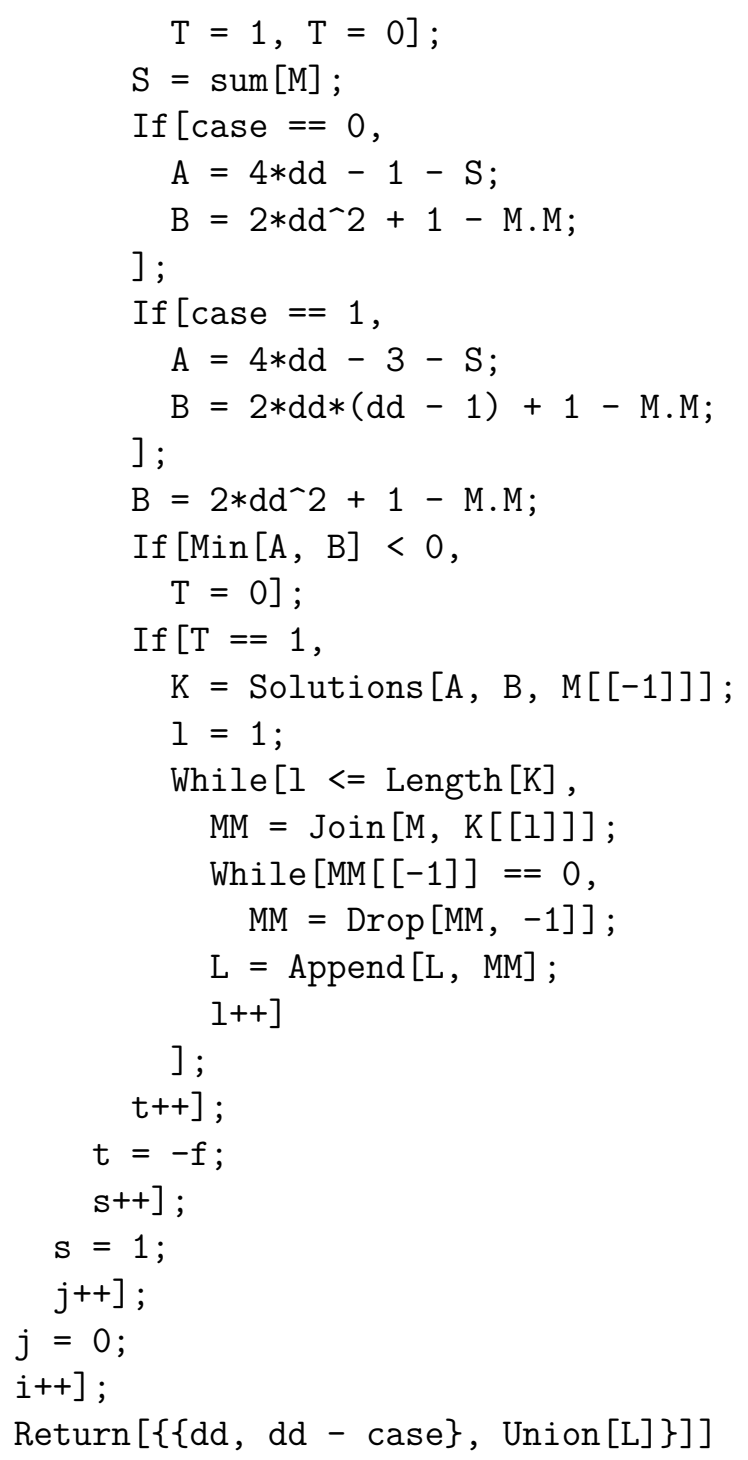

As in [MS], the module InterSol $[\mathrm{k}, \mathrm{d}, \mathrm{c}]$ reduces the number of candidates given by the code Prelist $[\mathrm{k}, \mathrm{d}, \mathrm{c}]$. As before, $c=0$ in the case of a class of the form $(d, d ; m)$ and $c=1$ for a class of the form $(d, d-1 ; m)$.

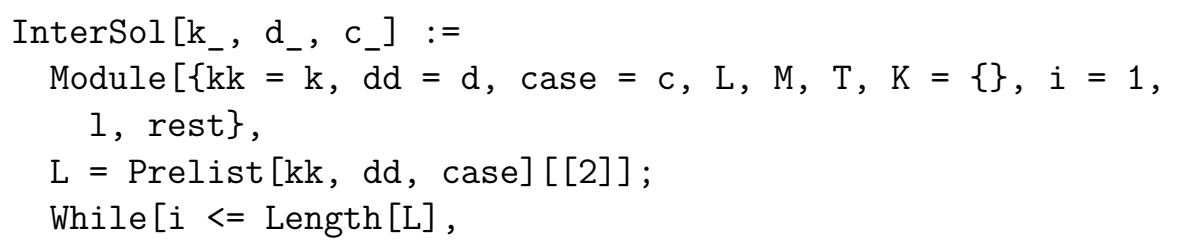




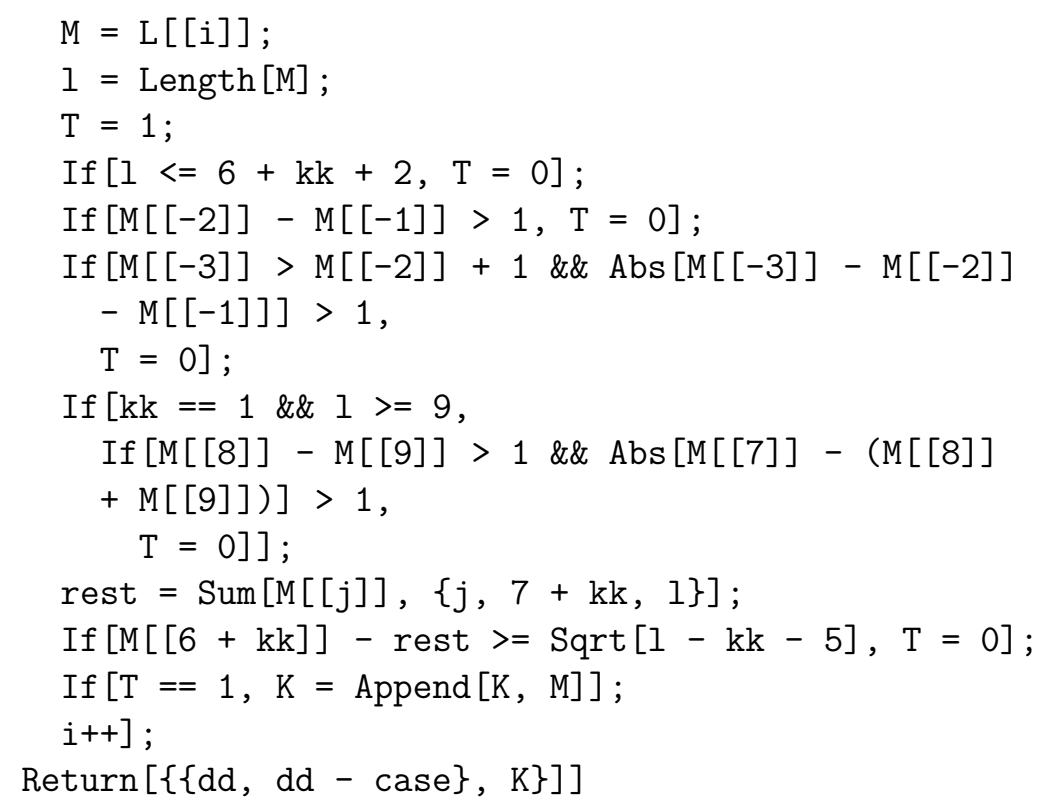

Finally, we collect all the solutions for $d \leqslant D$ in InterSolLess $1[\mathrm{k}, \mathrm{D}]$.

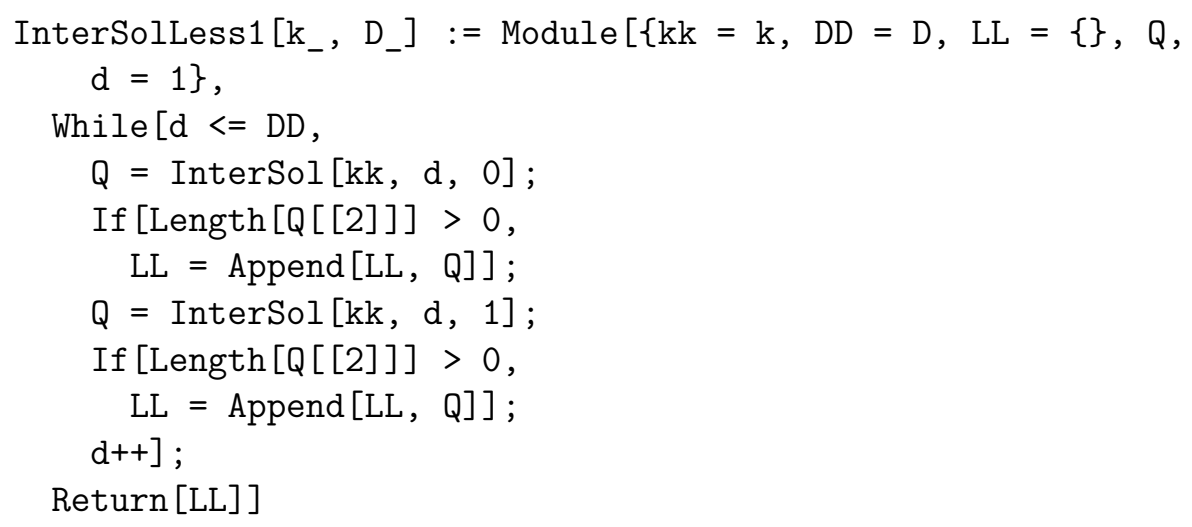

A.2.2. Finding obstructive classes $(d, e ; m)$ with $m_{1} \neq m_{6}$. The code InterSolLess2[k, D] gives for $k \in\{1, \ldots, 7\}$ and a natural number $D$, a finite list of vectors $(d, e ; m)$ with $d \leqslant D$ and $m_{1} \neq m_{6}$ which can potentially be obstructive at some $a \in] 6 \frac{1}{k+1}, 6 \frac{1}{k}[$. By Corollary 4.15 , if a class $(d, e ; m) \in \mathcal{E}$ with $m_{1} \neq m_{6}$ is obstructive at some $a \in[6,7$ [, then necessarily $d=e$. Moreover, Lemma 4.14 shows that either $m_{1}-1=m_{2}=\cdots=m_{6}$ or $m_{1}=\cdots=m_{5}=m_{6}+1$. Notice that the first terms of the weight expansion of some $a \in] 6 \frac{1}{k+1}, 6 \frac{1}{k}\left[\right.$ are $\left(1^{\times 6} ;(a-6)^{\times k}, \ldots\right)$. Thus the vector $m$ is either of the form $\left(M+1, M^{\times 5}, m^{\times k}, \ldots\right)$ or of the form $\left(M,(M-1)^{\times 5}, m^{\times k}, \ldots\right)$. To 
find the vectors $m$ of the form $\left(M+1, M^{\times 5}, m^{\times k}, \ldots\right)$, we vary $M$ and $m \leqslant$ $M$ as long as $(M+1)+5 M+k m \leqslant 4 d-1$ and $(M+1)^{2}+5 M^{2}+k m^{2} \leqslant$ $2 d^{2}+1$ and then use the code Solutions[a,b] from [MS] to find the solutions of the equations

$$
\begin{aligned}
& \sum m_{i}=4 d-1-((M+1)+5 M+k m), \\
& \left.\sum m_{i}^{2}=2 d^{2}-1-(M+1)^{2}+5 M^{2}+k m^{2}\right) .
\end{aligned}
$$

The case of a solution vector $m$ of the form $\left(M,(M-1)^{\times 5}, m^{\times k}, \ldots\right)$ is then treated similarly.

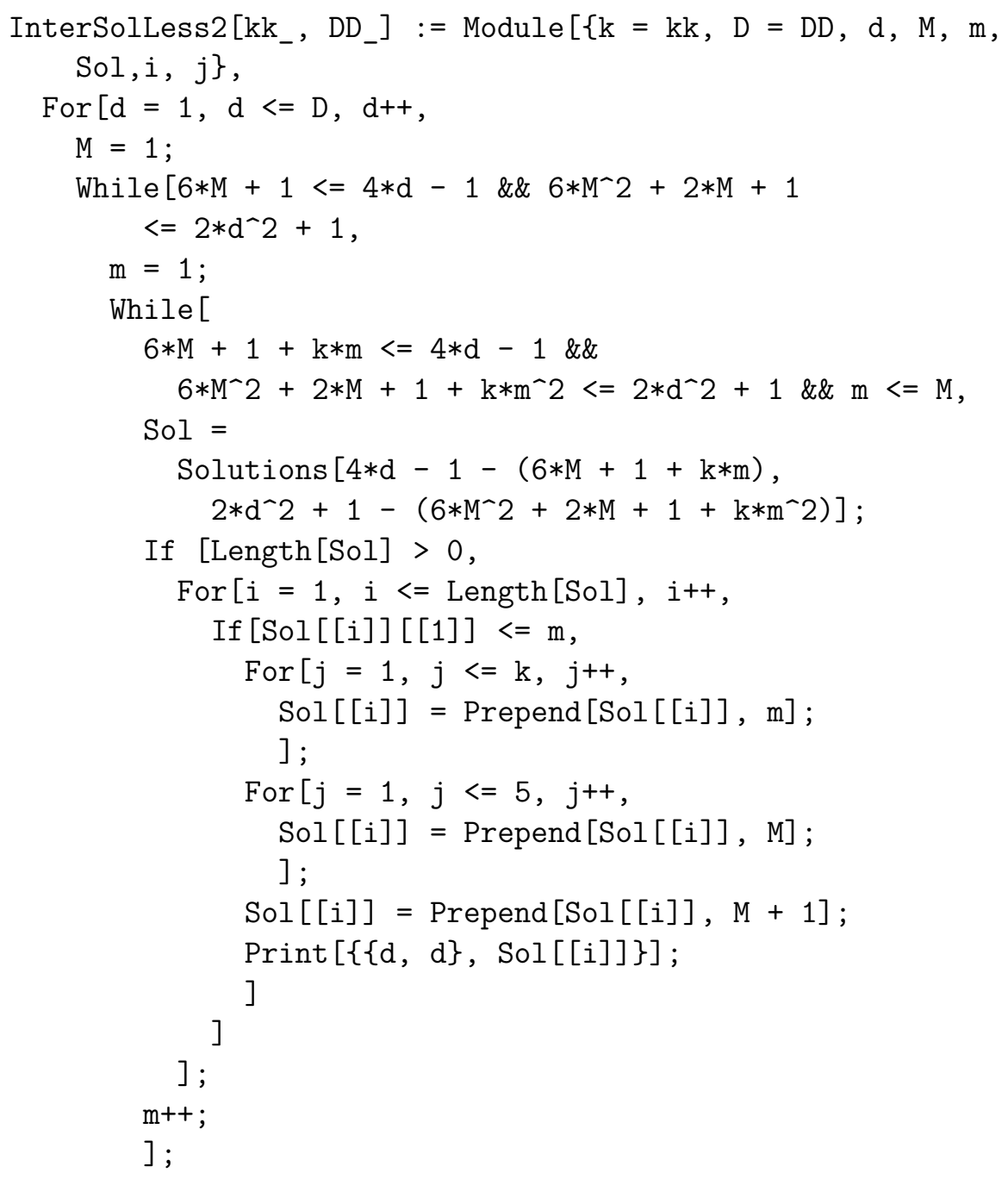




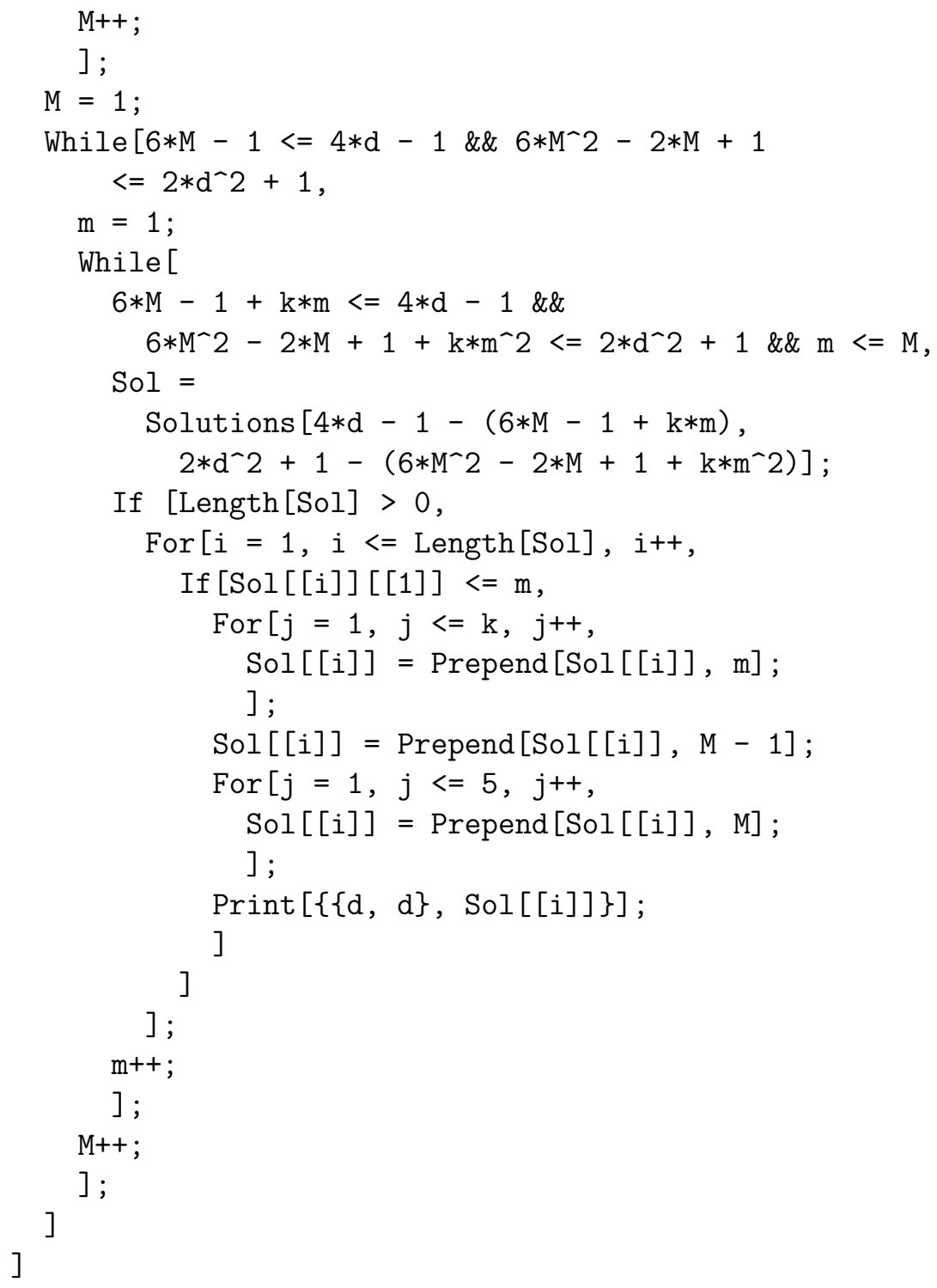

\section{References}

[B1] P. Biran, Symplectic packing in dimension 4. Geom. Funct. Anal., 7 (1997), 420-437.

[B2] P. Biran, A stability property of symplectic packing. Invent. Math., 136 (1999), 123-155. 
[BuH1] O. Buse and R. Hind, Symplectic embeddings of ellipsoids in dimension greater than four. Geom. Topol., 15 (2011), 2091-2110.

[BuH2] O. Buse and R. Hind, Ellipsoid embeddings and symplectic packing stability. Compos. Math., 149 (2013), 889-902.

$[\mathrm{BuP}]$ O. Buse and M. Pinsonnault, Packing numbers of rational ruled 4manifolds. Journal of Symplectic Geometry, 11 (2013), 269-316.

[G] M. Gromov, Pseudoholomorphic curves in symplectic manifolds. Invent. Math., 82 (1985), 307-347.

[H1] M. Hutchings, Quantitative embedded contact homology. J. Differential Geom., 88 (2011), 231-266.

[H2] M. Hutchings, Recent progress on symplectic embedding problems in four dimensions. Proc. Natl. Acad. Sci. USA, 108 (2011), 8093-8099.

[K] Y. Karshon, Appendix to [MP]. Invent. Math., 115 (1994), 431-434.

[LMS] J. Latschev, D. McDuff and F. Schlenk, The Gromov width of 4dimensional tori. arXiv:1111.6566.

[LiLi] B.-H. Li and T.-J. Li, Symplectic genus, minimal genus and diffeomorphisms. Asian J. Math., 6 (2002), 123-144.

[LiLiu] T.-J. Li and A. K. Liu, Uniqueness of symplectic canonical class, surface cone and symplectic cone of 4 -manifolds with $b^{+}=1$. J. Differential Geom., 58 (2001), 331-370.

[M1] D. McDuff, From symplectic deformation to isotopy. In: Topics in symplectic 4-manifolds (Irvine, CA, 1996), 85-99, First Int. Press Lect. Ser., I, Int. Press, Cambridge, MA, 1998.

[M2] D. McDuff, Symplectic embeddings of 4-dimensional ellipsoids. J. Topol., 2 (2009), 1-22.

[M3] D. McDuff, The Hofer conjecture on embedding symplectic ellipsoids. J. Differential Geom., 88 (2011), 519-532.

[MP] D. McDuff and L. Polterovich, Symplectic packings and algebraic geometry. Invent. Math., 115 (1994), 405-429.

[MS] D. McDuff and F. Schlenk, The embedding capacity of 4-dimensional symplectic ellipsoids. Annals of Math., 175 (2012), 1191-1282.

[O] E. Opshtein, Symplectic packings in dimension 4 and singular curves. arXiv: 1110.2385. 
[S1] F. Schlenk, Volume preserving embeddings of open subsets of $\mathbb{R}^{n}$ into manifolds. Proc. Amer. Math. Soc., 131 (2003), 1925-1929.

[S2] F. Schlenk, Embedding problems in symplectic geometry. de Gruyter Expositions in Mathematics 40, Walter de Gruyter, Berlin, 2005.

[T] L. Traynor, Symplectic packing constructions. J. Differential Geom., 42 (1995), 411-429.

Conférence universitaire de Suisse occidentale Faubourg

DE L'HÔPITAL 19

CP 2133 CH-2001 Neuchâtel, Switzerland

E-mail address: david.frenkel@unine.ch

Université DE NeUChÂTEL, Institut DE MATHÉMATIQUES

Rue Emile-Argand 11, 2000 Neuchâtel, Switzerland

E-mail address: dorothee.mueller@unine.ch

Received OCtober 15, 2012 
